JOURNAL OF THE

AMERICAN MATHEMATICAL SOCIETY

Volume 26, Number 2, April 2013, Pages 451-510

S 0894-0347(2012)00757-7

Article electronically published on November 20, 2012

\title{
LOGARITHMIC GROMOV-WITTEN INVARIANTS
}

\author{
MARK GROSS AND BERND SIEBERT
}

\section{Contents}

Introduction

1. Stable log maps

1.1. Log smooth curves 454

1.2. Stable log maps 456

1.3. The category GS

1.4. The standard log point and tropical curves 460

1.5. The basicness condition 464

2. Algebraicity 470

2.1. The stack of stable log maps $\quad 470$

2.2. Representability of spaces of log morphisms 472

3. Boundedness 476

3.1. Finiteness of combinatorial types 476

3.2. Stable log maps of constant type and boundedness 481

4. Stable reduction 484

4.1. Extension on the level of ghost sheaves 484

4.2. The $\log$ structure on the base 486

4.3. Extension of the log morphism 490

5. Log Gromov-Witten invariants 491

6. The relationship with expanded degenerations 494

7. Examples

Appendix A. The log stack of prestable curves

Appendix B. Tropicalization of a log space

\begin{tabular}{ll} 
Acknowledgements & 509 \\
\hline
\end{tabular}

\begin{tabular}{ll} 
References & 509 \\
\hline
\end{tabular}

\section{INTRODUCTION}

The purpose of this paper is the development of a general theory of GromovWitten invariants in logarithmically smooth situations. Concrete examples of such situations are non-singular projective varieties with a normal crossing divisor, central fibres of semi-stable degenerations, or toroidal pairs. They occur naturally in Gromov-Witten theory in imposing constraints, in dimensional reduction and in degeneration situations. The easiest, and already quite useful special case is

Received by the editors March 16, 2011 and, in revised form, August 26, 2011, and July 30, 2012 .

2010 Mathematics Subject Classification. Primary 14D20, 14N35.

This work was partially supported by NSF grants 0505325 and 0805328 .

(C)2012 American Mathematical Society Reverts to public domain 28 years from publication 
Gromov-Witten invariants relative to a smooth divisor. The first treatments used symplectic techniques $[\mathrm{LiRu}, \mathrm{IoPa}$. Algebraically a direct approach for very ample divisors is possible $\mathrm{Ga}$, the much more complicated general case is due to $\mathrm{Jun} \mathrm{Li}$ [Li1], Li2]. Any of the general approaches use a geometrically beautiful, but technically unpleasant change of target space with the purpose of avoiding irreducible components mapping into the divisor. As an application these authors also developed decomposition formulas of absolute Gromov-Witten invariants into sums of relative Gromov-Witten invariants under a semistable degeneration of the target variety into two components intersecting along a smooth divisor (symplectically, a symplectic cut).

While Jun Li's theory was under development the second named author suggested that a far more general and potentially technically simpler theory should be possible using abstract log geometry [Si2]. This paper is the late realization of this proposal. While several problems could be solved back then, notably stable reduction and the construction of virtual fundamental classes, there remained a fundamental problem of selecting the natural ("basic") log structure on the base scheme of a family of stable log maps. Without a notion of basicness it is virtually impossible to prove algebraicity and quasi-compactness of the relevant moduli stack. With hindsight one can say that the suggestion of log Gromov-Witten theory was premature in 2001 because two major ingredients were only just developing, tropical geometry on the one hand and more powerful techniques for the treatment of $\log$ moduli problems on the other hand. In fact, the essential insight for the notion of basicness came from a discussion of the authors in August 2008 and was guided by our understanding of moduli of tropical curves and their relation to log geometry. As for log moduli problems, Olsson's algebraic stack of fine log structures $\mathrm{Ol3}$ ] is crucial to prove algebraicity of our stack of basic stable log maps, and Olsson's many other works on problems and applications of log geometry served as a blueprint at many stages of this work.

The main concept is that of a basic stable log map (Definition 1.6 and Definition 1.20). A stable log map is just a stable map with all arrows defined in the category of log schemes and the condition on the domain to be pre-stable replaced by $\log$ smoothness. We show that stable log maps to a fixed log scheme $X=\left(\underline{X}, \mathcal{M}_{X}\right)$ form an algebraic stack $\tilde{\mathscr{M}}(X)$ (Theorem 2.4). The main ingredients in this proof are Olsson's algebraic stack $\mathcal{L}$ og of fine log schemes, the understanding of pre-stable curves from the log point of view [Kf], and a representability result for spaces of log morphisms in relative situations (Proposition 2.9). The stack $\tilde{\mathscr{M}}(X)$ is far too large because it allows arbitrary log structures on the base of a family of stable log maps. The notion of basicness (Definition 1.20) selects a universal choice that interacts well with geometry. Basicness only depends on the log morphism on the level of ghost sheaves. The relation to tropical geometry comes from pulling back to the standard log point $(\$ 1.4)$. In situations where tropical geometry applies, the moduli space of associated tropical curves is the dual of the basic monoid (the stalk of the ghost sheaf of the log structure on the base). In general, this picture is a bit problematic, but there is still a way to visualize stable log maps by (families of) tropical curves with values in a topological, piecewise polyhedral space $\operatorname{Trop}(X)$ that is canonically associated to $X$ (Appendix B). In any case, basicness selects an open substack $\mathscr{M}(X)$ of $\tilde{\mathscr{M}}(X)$ which now also has a separated diagonal. It also comes with a natural log structure. This is the first main result (stated as 
Proposition 2.3 and Corollary 2.6 in the text). We work over a fixed fine saturated $\log$ scheme $S=\left(\underline{S}, \mathcal{M}_{S}\right)$.

Theorem 0.1. Let $X=\left(\underline{X}, \mathcal{M}_{X}\right)$ be a fine saturated log scheme. Then the stack $\mathscr{M}(X / S)$ of basic stable log maps to $X$ over $S$ is an algebraic log stack locally of finite type over $\underline{S}$. The forgetful morphism $\mathscr{M}(X / S) \rightarrow \mathbf{M}(\underline{X} / \underline{S})$ to the stack of ordinary stable maps over $\underline{S}$ is representable.

A basic problem, which we could not solve in complete generality, is to identify quasi-compact substacks of $\mathscr{M}(X / S)$. Natural conditions concern the classical conditions on genus, number of marked points and homology class of the underlying ordinary stable map, plus logarithmic conditions at the marked points similar to the orders of tangency with the divisor in the classical situation. Denoting $\mathscr{M}(X / S, \beta)$ the substack of $\mathscr{M}(X / S)$ fulfilling such a set of conditions, a stratawise approach reduces the question of boundednesss to a generally subtle question in convex geometry (Theorem 3.12), summarized in the concept of combinatorial finiteness of a class $\beta$ (Definition 3.3). We have complete results provided the pull-back of $\overline{\mathcal{M}}_{X}^{\mathrm{gp}} \otimes_{\mathbb{Z}} \mathbb{Q}$ to any stable log map is globally generated, that is, if any germ of a section is the restriction of a global section. Then any $\beta$ is combinatorially finite (Theorem 3.9). This criterion holds in the important special cases of genus 0 , of simple normal crossing divisors and in toric situations. We also have boundedness without further conditions as long as the dual intersection graph of the domain has genus at most one (Theorem 3.11) and in certain favourable stratawise situations (Theorem 3.10). In concrete situations one can check combinatorial finiteness by examining a finite list of ordinary stable maps.

Arguably the most difficult single result in this paper is stable reduction (Theorem (4.1), which requires a solid understanding of the interaction between the basicness condition and geometry. Taken together with boundedness we obtain the second main result (Corollary 4.2 in the text).

Theorem 0.2. For $\underline{X}$ projective over $\underline{S}$ and $\beta$ a combinatorially finite class of stable log maps, $\mathscr{M}(X / S, \beta)$ is proper over $\underline{S}$.

The construction of a virtual fundamental class on $\mathscr{M}(X / S, \beta)$ now is completely straightforward via the approach of [BeFa] using Olsson's log cotangent complex Ol3, as already worked out by Kim [Ki]. The details are given in Section [5. One can then define log Gromov-Witten invariants in the usual way by pairing with cohomology classes on $\underline{X}$ via evaluation at the marked points. This construction then leads to our third main result.

Theorem 0.3. Assume that $X$ is smooth over $S$. Then under the assumptions of Theorem 0.2 there exists a virtual fundamental class $\llbracket \mathscr{M}(X / S, \beta) \rrbracket$ leading to log Gromov-Witten invariants with the expected properties.

Among the expected properties are compatibility with base change, equality with the ordinary virtual fundamental class for trivial log structures and with the usual fundamental class in unobstructed situations, and many more that follow by strict analogy with the ordinary case.

While this work was in progress we learned of ongoing work of Abramovich, Chen and coworkers aiming at carrying out our program in the special case that there exists a surjection $\underline{\mathbb{N}}^{r} \rightarrow \overline{\mathcal{M}}_{X}$ ( $\mathrm{Ch}$, ACGM], AbCh] . 
Conventions. We work in the categories of schemes of finite type over a field $\mathbb{k}$ of characteristic 0 and of fine saturated log schemes $[\mathrm{Kk}$ over a fixed base log scheme $S$, itself fine and saturated. Our standard notation for log schemes is $X=\left(\underline{X}, \mathcal{M}_{X}\right)$, but we do not underline ordinary schemes that we do not want to endow with a $\log$ structure unless there is a chance of confusion. Similarly, for a morphism of log schemes the notation is $f=\left(f, f^{b}\right)$. The ghost sheaf (also called characteristic) of a $\log$ structure $\mathcal{M}$ is denoted $\overline{\mathcal{M}}:=\mathcal{M} / \mathcal{O}^{\times}$. We use multiplicative notation for $\mathcal{M}$ and additive notation for $\overline{\mathcal{M}}$. Throughout $X$ is a $\log$ scheme over $S$ such that $\mathcal{M}_{S}$, $\mathcal{M}_{X}$ and the structure morphism $\left(\underline{X}, \mathcal{M}_{X}\right) \rightarrow\left(\underline{S}, \mathcal{M}_{S}\right)$ are defined in the Zariski topology. To have a good theory of ordinary stable maps at our disposal we require $\underline{X}$ to be quasi-projective over $\underline{S}$. If $Y$ is a scheme (algebraic space, stack), then $|Y|$ denotes the set of geometric points of $Y$ endowed with the Zariski topology; see [Kt, II.6 or LaMB], Ch.5. We use overlined symbols to denote elements of $|Y|$; in particular, if $y$ is a scheme-theoretic point of $Y$, then $\bar{y}$ is a geometric point with image $y$. A toric monoid is a fine, saturated, torsion-free monoid without nontrivial invertibles. For a monoid $P$ we write $P^{\vee}:=\operatorname{Hom}(P, \mathbb{N})$ for the dual in the category of monoids, $P^{\text {gp }}$ for the associated abelian group and $P^{*}:=\operatorname{Hom}(P, \mathbb{Z})$.

\section{Stable LOG MAPS}

1.1. Log smooth curves. In this subsection we work in the absolute situation $S=$ Spec $\mathbb{k}$ with the trivial log structure. A logarithmic version of the theory of (pre-)stable curves of Deligne, Mumford and Knudsen [DeMu, [Kd] has been developed by F.Kato [Kf], see also Ol1]. The starting point is the following result on the structure of $\log$ smooth curves.

Theorem 1.1 ([Kf], p. 222). Let $\pi: C \rightarrow W$ be a smooth and integral morphism of fine saturated log schemes such that every geometric fibre is a reduced curve. Assume that $\underline{W}=\operatorname{Spec} A$ for $(A, \mathfrak{m})$ a strictly Henselian local ring. Let $0 \in \underline{W}$ be the closed point, $Q=\overline{\mathcal{M}}_{W, 0}$ and $\sigma: Q \rightarrow A$ a chart for the log structure on $W$. Then étale locally $C$ is isomorphic to one of the following log schemes $V$ over $W$.

(i) $\operatorname{Spec}(A[z])$ with the log structure induced from the homomorphism

$$
Q \longrightarrow \mathcal{O}_{V}, \quad q \longmapsto \sigma(q) .
$$

(ii) $\operatorname{Spec}(A[z])$ with the log structure induced from the homomorphism

$$
Q \oplus \mathbb{N} \longrightarrow \mathcal{O}_{V}, \quad(q, a) \longmapsto z^{a} \sigma(q) .
$$

(iii) $\operatorname{Spec}(A[z, w] /(z w-t))$ with $t \in \mathfrak{m}$ and with the log structure induced from the homomorphism

$$
Q \oplus_{\mathbb{N}} \mathbb{N}^{2} \longrightarrow \mathcal{O}_{V}, \quad(q,(a, b)) \longmapsto \sigma(q) z^{a} w^{b} .
$$

Here $\mathbb{N} \rightarrow \mathbb{N}^{2}$ is the diagonal embedding and $\mathbb{N} \rightarrow Q, 1 \mapsto \rho_{q}$ is some homomorphism uniquely defined by $C \rightarrow W$. Moreover, $\rho_{q} \neq 0$.

In this list, the morphism $C \rightarrow W$ is represented by the canonical maps of charts $Q \rightarrow Q, Q \rightarrow Q \oplus \mathbb{N}$ and $Q \rightarrow Q \oplus_{\mathbb{N}} \mathbb{N}^{2}$, respectively.

Case (i) deals with smooth points of the central fibre where the log structure comes entirely from the base while (iii) covers the situation in a neighbourhood of the degeneracy locus of $\pi$. To interpret case (ii) observe that the preimage of $z=0$ defines a section of $\pi$ on an étale neighbourhood of $x$. This should be viewed as the section defining a marked point. Let $\Gamma \subset \underline{C}$ be the image of this section and 
$\iota: \underline{C} \backslash \Gamma \hookrightarrow \underline{C}$ the inclusion. Then the $\log$ structure of $C$ near $x$ is the sum of the $\log$ structure of the base and the $\log$ structure $\iota_{*}\left(\mathcal{O}_{\underline{C} \backslash \Gamma}^{\times}\right) \cap \mathcal{O}_{\underline{C}}$ associated to $\Gamma$.

Remark 1.2. (1) The monoid $Q$ together with $\rho_{q} \in Q$ for the nodes $q \in \underline{C}$ determine the $\log$ structure on the closed fibre $\underline{C}_{0}$ and the morphism to the log point $(\operatorname{Spec}(A / \mathfrak{m}), Q)$ on the level of ghost sheaves. In fact, let $\bar{\eta}$ be the generic point of the branch of $\underline{C}_{0}$ defined by $w=0$ in (iii). Then at $\bar{\eta}$ the element $z^{a}$ becomes invertible. Hence the compatibility of the charts in (i) and (iii) implies that the generization map $\overline{\mathcal{M}}_{C_{0}, \bar{q}} \longrightarrow \overline{\mathcal{M}}_{C_{0}, \bar{\eta}}$ equals

$$
Q \oplus_{\mathbb{N}} \mathbb{N}^{2} \longrightarrow Q, \quad(m,(a, b)) \longmapsto m+b \cdot \rho_{q} .
$$

These generization maps together with one copy of $\mathbb{N}$ at each of the special points in (ii) define $\overline{\mathcal{M}}_{C_{0}}$ and the structure homomorphism $Q \rightarrow \Gamma\left(\underline{C}_{0}, \overline{\mathcal{M}}_{C_{0}}\right)$ uniquely up to unique isomorphism.

Note that if $\underline{C}$ has non-normal irreducible components, then $\mathcal{M}_{C}$ is only defined over the étale site. Still, $\overline{\mathcal{M}}_{C}$ can be described completely by generization maps, the only difference now being that at a node $q \in \underline{C}$ in the closure of only one generic point $\eta$ there are two generization maps $\overline{\mathcal{M}}_{C, \bar{q}} \rightarrow \overline{\mathcal{M}}_{C, \bar{\eta}}$.

(2) At a node $q \in \underline{C}_{0}$ the two generization maps $Q \oplus_{\mathbb{N}} \mathbb{N}^{2} \rightarrow Q$ define the homomorphism

$$
\iota: Q \oplus_{\mathbb{N}} \mathbb{N}^{2} \longrightarrow Q \times Q, \quad(m,(a, b)) \longmapsto\left(m+a \cdot \rho_{q}, m+b \cdot \rho_{q}\right) .
$$

If $\iota((m,(a, b)))=0$, then $m+a \rho_{q}=m+b \rho_{q}=0$, and hence $a=b$ since $\rho_{q} \neq 0$. In view of the definition of $Q \oplus_{\mathbb{N}} \mathbb{N}^{2}$ this implies

$$
(m,(a, b))=(m,(a, a))=\left(m+a \rho_{q},(0,0)\right)=0 .
$$

Thus $\iota$ exhibits $Q \oplus_{\mathbb{N}} \mathbb{N}^{2}$ as a submonoid of $Q \times Q$ :

$$
Q \oplus_{\mathbb{N}} \mathbb{N}^{2} \simeq\left\{\left(m_{1}, m_{2}\right) \in Q \times Q \mid m_{1}-m_{2} \in \mathbb{Z} \rho_{q} \text { in } Q^{\mathrm{gp}}\right\} \subset Q \times Q .
$$

In fact, if $m_{1}-m_{2}=\alpha \rho_{q}$, then

$$
\left(m_{1}, m_{2}\right)= \begin{cases}\iota\left(m_{2},(\alpha, 0)\right), & \alpha \geq 0, \\ \iota\left(m_{1},(0,-\alpha)\right), & \alpha<0 .\end{cases}
$$

The theorem suggests the following generalization of the notion of marked (pre-) stable curves to log geometry.

Definition 1.3. A pre-stable (marked) log curve over $W$ is a pair $(C / W, \mathbf{x})$ consisting of a proper log smooth and integral morphism $\pi: C \rightarrow W$ of fine saturated $\log$ schemes over $S$ together with a tuple of sections $\mathbf{x}=\left(x_{1}, \ldots, x_{l}\right)$ of $\underline{\pi}$, such that every geometric fibre of $\pi$ is a reduced and connected curve, and if $U \subset \underline{C}$ is the non-critical locus of $\pi$, then $\left.\overline{\mathcal{M}}_{C}\right|_{U} \simeq \underline{\pi}^{*} \overline{\mathcal{M}}_{W} \oplus \bigoplus_{i} x_{i *} \mathbb{N}_{\underline{W}}$.

A pre-stable log curve is stable if forgetting the log structure leads to an ordinary stable curve.

Remark 1.4. (1) The underlying morphism of a pre-stable log curve is flat and its geometric fibres have at most ordinary double points. The underlying morphism of schemes of a pre-stable log curve is hence an ordinary pre-stable curve.

(2) The condition on $\overline{\mathcal{M}}_{C}$ says that the sections $x_{i}$ label precisely the special nonnodal points occurring in Theorem 1.1(ii). A straightforward generalization would only label a subset of the special non-nodal points. 
We sometimes deal with diagrams of spaces endowed with fine sheaves of monoids that look like they are the diagrams of ghost sheaves of a pre-stable log curve. For later reference we cast this situation into the following definition.

Definition 1.5. Let $(\underline{\pi}: \underline{C} \rightarrow \underline{W}, \mathbf{x})$ be an ordinary pre-stable curve over a scheme $\underline{W}$ and let $\underline{\pi}^{*} \overline{\mathcal{M}}_{W} \rightarrow \overline{\mathcal{M}}_{C}$ be a morphism of fine sheaves of monoids on $\underline{C}$ and $\underline{W}$, respectively. We say that $\left(\left(\underline{C}, \overline{\mathcal{M}}_{C}\right) /\left(\underline{W}, \overline{\mathcal{M}}_{W}\right), \mathbf{x}\right)$ has the structure of a pre-stable $\log$ curve on the level of ghost sheaves if for every geometric point $\bar{w}: \operatorname{Spec} \kappa \rightarrow \underline{W}$ the situation is described by Remark $1.2(1)$. In particular, writing $Q=\overline{\mathcal{M}}_{W, \bar{w}}$, then for each node $q \in \underline{C}_{\bar{w}}$ there is an element $\rho_{q} \in Q$ such that $\left.\bar{M}_{W}\right|_{\underline{C}_{\bar{w}}}$ is isomorphic to the sheaf with stalks $Q \oplus_{\mathbb{N}} \mathbb{N}^{2}$ (with $1 \in \mathbb{N}$ mapping to $\rho_{q} \in Q$ and to $(1,1) \in \mathbb{N}^{2}$ ) at $q$, with stalks $Q \oplus \mathbb{N}$ at the marked points, and with stalks $Q$ at all other points; the generization maps and the morphism $\left.\overline{\mathcal{M}}_{W, \bar{w}} \rightarrow \overline{\mathcal{M}}_{C}\right|_{\underline{w}_{\bar{w}}}$ are as described in Remark 1.2(1).

F. Kato also introduces the notion of basic log structure of a marked stable curve (骨], p. 227f). A simple way to think about this concept is as follows. Let $\left(\underline{\pi}: \underline{C} \rightarrow \underline{W}, \mathbf{x}=\left(x_{1}, \ldots, x_{k}\right)\right)$ be a stable marked curve. Locally with respect to the base, $(\underline{C} / \underline{W}, \mathbf{x})$ is the pull-back by a morphism $\underline{W} \rightarrow \underline{T}$ of a pre-stable marked curve $(\underline{q}: \underline{U} \rightarrow \underline{T}, \mathbf{y})$ that is formally versal at any point of $\underline{T}$. Versality implies that the image of the subspace of $\underline{U}$ defined by the first Fitting ideal of $\Omega_{\underline{U} / \underline{T}}^{1}$ is a normal crossings divisor $D \subset \underline{T}$. This divisor is the scheme theoretic version of the subset of $\underline{T}$ parametrizing singular curves. We endow $\underline{T}$ and $\underline{U}$ with the $\log$ structures associated to the divisors $D$ in $\underline{T}$ and $q^{-1}(D)$ and the divisor of marked points in $\underline{U}$, respectively. The basic $\log$ structure on $\underline{C} \rightarrow \underline{W}$ is then obtained by the pull-back via $\underline{W} \rightarrow \underline{T}$. Globally one obtains an étale descent datum for $\mathcal{M}_{C}$, $\mathcal{M}_{W}$ and for the morphism $\pi^{*} \mathcal{M}_{W} \rightarrow \mathcal{M}_{C}$. Note also that at a geometric point $\bar{w}$ of $\underline{W}$ there is an isomorphism $\overline{\mathcal{M}}_{W, \bar{w}} \simeq \mathbb{N}^{r}$ where $r$ is the number of double points of $\underline{C}_{\bar{w}}$, which by versality equals the number of branches of $D$ at the image of $\bar{w}$ in $\underline{T}$.

The existence of basic log structures on stable curves makes it possible to endow the stacks $\mathbf{M}_{g, k}$ of $l$-marked stable curves with a logarithmic structure [Kf], p. 230f. This means ( $\mathrm{Ol2}$, Definition 5.1 and Corollary 5.8) that there exists a factorization

$$
\mathbf{M}_{g, k} \longrightarrow(\mathrm{Log}) \longrightarrow(\mathrm{Sch})
$$

of the functor defining the stack $\mathbf{M}_{g, k}$. Here $(\log )$ is the category of fine saturated $\log$ schemes with strict morphisms. The first arrow maps a family $(\underline{C} / \underline{W}, \underline{\mathbf{x}})$ of $k$-marked stable curves of genus $g$ to its base scheme $\underline{W}$ endowed with the basic $\log$ structure. The factorization (1.2) endows $\mathbf{M}_{g, k}$ with a log structure, defining a $\log$ stack $\mathscr{M}_{g, k}$. Of course, not every stable log curve carries the basic log structure of the underlying stable marked curve. The log structure can rather always be obtained from the basic log structure by a unique base change inducing the identity on the underlying spaces ([Kf], Proposition 2.1). See also [Ol1], Ch.5, for an extended treatment.

Analogous statements hold for pre-stable curves, leading to the log algebraic stack $\mathscr{M}$, an Artin stack (see Appendix A).

1.2. Stable log maps. We now turn to the main concept of this paper, a logarithmic version of the notion of stable map. Recall that $X$ is a log scheme over $S$, with $\log$ structures defined on the Zariski sites. 
Definition 1.6. A log curve over $X$ with base $W$ is a pre-stable marked log curve $(C / W, \mathbf{x})$ (Definition [1.3) together with a morphism $f: C \rightarrow X$ fitting into a commutative diagram of $\log$ schemes

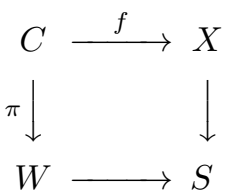

A $\log$ curve over $X$ is a stable log map if for every geometric point $\bar{w} \rightarrow \underline{W}$ the restriction of $f$ to the underlying pre-stable marked curve belonging to $\underline{C}_{\bar{w}} \rightarrow \bar{w}$ is an ordinary stable map. The notation is $(C / W, \mathbf{x}, f)$ with the morphisms $\pi: C \rightarrow$ $W$ and $W \rightarrow S$ usually understood.

A morphism of stable log maps

$$
\Phi:\left(C_{1} / W_{1}, \mathbf{x}_{1}, f_{1}\right) \longrightarrow\left(C_{2} / W_{2}, \mathbf{x}_{2}, f_{2}\right)
$$

is a cartesian diagram of $\log$ curves $\Phi: C_{1} / W_{1} \rightarrow C_{2} / W_{2}$ over $S$ with $W_{1} \rightarrow W_{2}$ strict and such that $f_{1}=f_{2} \circ \Phi$ and $\mathbf{x}_{2}=\underline{\Phi} \circ \mathbf{x}_{1}$.

The category of stable log maps thus obtained is denoted $\tilde{\mathscr{M}}(X / S)$ or just $\tilde{\mathscr{M}}(X)$.

Let $\kappa$ be a field. For a toric monoid $Q$ with $Q^{\times}=\{0\}$ denote by $(\operatorname{Spec} \kappa, Q)$ the associated logarithmic point, that is, Spec $\kappa$ with $\log$ structure

$$
Q \times \kappa^{\times} \longrightarrow \kappa, \quad(q, a) \longmapsto \begin{cases}0, & q \neq 0, \\ a, & q=0 .\end{cases}
$$

Now let $\underline{C}$ be a pre-stable curve over $\kappa$, and assume given a fine saturated log structure $\alpha: \mathcal{M} \rightarrow \mathcal{O}_{C}$ on the Zariski site of $\underline{C}$. For the application to stable $\log$ maps we will only consider $\mathcal{M}=\underline{f}^{*} \mathcal{M}_{X}$ for some $\underline{f}: \underline{C} \rightarrow \underline{X}$, but this is not relevant for the following discussion. In any case, this $\log$ structure $\mathcal{M}$ can be rather arbitrary and certainly does not need to be smooth for any log structure on Spec $\kappa$. We need to understand diagrams

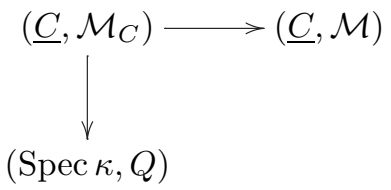

with left-hand vertical arrow smooth, for some toric monoid $Q$. In the simplest case of trivial $\mathcal{M}$ we are in the situation recalled in Section 1.1. Thus in this case there is a universal such diagram with $Q=\mathbb{N}^{r}$ and $r$ the number of nodes of $C$, in the sense that any other diagram is obtained by unique pull-back. We will see that there is a similarly universal object in complete generality. The essential step is the characterization of a universal such diagram on the level of ghost sheaves. This is the object of the next subsection.

1.3. The category GS. Let $\overline{\mathcal{M}}$ be a fine saturated sheaf on a pre-stable curve $(\underline{\pi}: \underline{C} \rightarrow \underline{W}, \mathbf{x})$. We consider the following category.

Definition 1.7. Let $\underline{\operatorname{GS}}(\overline{\mathcal{M}})$ (for "ghost sheaves") be the category with objects

$$
\left(\overline{\mathcal{M}}_{W}, \overline{\mathcal{M}}_{C}, \psi: \underline{\pi}^{*} \overline{\mathcal{M}}_{W} \rightarrow \overline{\mathcal{M}}_{C}, \varphi: \overline{\mathcal{M}} \rightarrow \overline{\mathcal{M}}_{C}\right),
$$


where $\overline{\mathcal{M}}_{W}$ and $\overline{\mathcal{M}}_{C}$ are fine sheaves on $\underline{W}$ and $\underline{C}$, respectively, and $\psi$ and $\varphi$ are loca 1 homomorphisms of fine (sheaves of) monoids. We require that $\psi$ endows $(\underline{C} / \underline{W}, \mathbf{x})$ with the structure of a pre-stable log curve over $\left(\underline{W}, \overline{\mathcal{M}}_{W}\right)$ on the level of ghost sheaves (Definition 1.5). Thus the objects of $\mathrm{GS}(\overline{\mathcal{M}})$ can alternatively be taken as diagrams

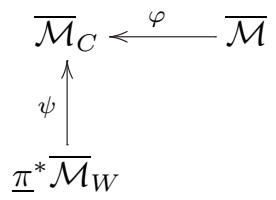

of fine saturated sheaves on $\underline{C}$ with local homomorphisms. A morphism

$$
\left(\overline{\mathcal{M}}_{W, 1}, \overline{\mathcal{M}}_{C, 1}, \psi_{1}, \varphi_{1}\right) \longrightarrow\left(\overline{\mathcal{M}}_{W, 2}, \overline{\mathcal{M}}_{C, 2}, \psi_{2}, \varphi_{2}\right)
$$

in $\underline{\operatorname{GS}}(\overline{\mathcal{M}})$ is given by a pair of homomorphisms $\overline{\mathcal{M}}_{W, 1} \rightarrow \overline{\mathcal{M}}_{W, 2}$ and $\overline{\mathcal{M}}_{C, 1} \rightarrow \overline{\mathcal{M}}_{C, 2}$ with the obvious compatibilities with $\psi_{i}$ and $\varphi_{i}, i=1,2$.

For the remainder of this subsection we restrict to the case $\underline{W}=\operatorname{Spec} \kappa$ for a field $\kappa$. In this case we write $Q$ for the stalk of $\overline{\mathcal{M}}_{W}$ and $\psi: Q \rightarrow \Gamma\left(\underline{C}, \overline{\mathcal{M}}_{C}\right)$. One central insight in this paper is the characterization of the connected components of $\underline{\operatorname{GS}}(\overline{\mathcal{M}})$ in this case by what we call the type of a stable log map. Even more useful, for each type we construct a universal object of the corresponding connected component (see Proposition 1.19 below). Saying that $\mathrm{GS}(\overline{\mathcal{M}})$ has a universal object essentially means that any two log enhancements of an ordinary stable map fit into one family, at least on the level of ghost sheaves.

Discussion 1.8. To introduce the concept of type let us reformulate Diagram (1.5) on the level of stalks. By the structure of stable log curves over $(\operatorname{Spec} \kappa, Q)$ there are three types of points $x$ on $\underline{C}$, depending on the stalks of $\overline{\mathcal{M}}_{C}$, as follows. Write $P_{x}:=\overline{\mathcal{M}}_{x}$

(i) $x=\eta$ is a generic point or a general closed point. Then $\overline{\mathcal{M}}_{C, \bar{\eta}}=Q$ and $\varphi$ defines a homomorphism

$$
\varphi_{\bar{\eta}}: P_{\eta} \longrightarrow Q
$$

(ii) $x=p$ is a marked point. Then $\overline{\mathcal{M}}_{C, \bar{p}}=Q \oplus \mathbb{N}$ with $\psi_{\bar{p}}$ inducing the inclusion of $Q$ as first factor. Then $\varphi_{\bar{p}}$ is determined by $\varphi_{\bar{\eta}}$ for $\bar{\eta}$ the generic point of the irreducible component containing $p$ together with

$$
u_{p}:=\operatorname{pr}_{2} \circ \varphi_{\bar{p}}: P_{p} \longrightarrow \mathbb{N} .
$$

(iii) $x=q$ is a node. Then $\overline{\mathcal{M}}_{C, \bar{q}} \simeq Q \oplus_{\mathbb{N}} \mathbb{N}^{2}$ with $\mathbb{N} \rightarrow Q, 1 \mapsto \rho_{q}$ and $\mathbb{N} \rightarrow \mathbb{N}^{2}$, $1 \mapsto(1,1)$. Let $\bar{\eta}_{1}, \bar{\eta}_{2}$ be the geometric generic points of the branches of $\underline{C}$

\footnotetext{
${ }^{1}$ A homomorphism of monoids $\varphi: P \rightarrow Q$ is called local if $\varphi^{-1}\left(Q^{\times}\right)=P^{\times}$or, equivalently, $\varphi^{-1}\left(Q \backslash Q^{\times}\right)=P \backslash P^{\times}$. Thus for the case of toric monoids this means $\varphi^{-1}(0)=0$.

${ }^{2}$ Recall that we assumed $\overline{\mathcal{M}}$ is a sheaf on the Zariski site, so $\overline{\mathcal{M}}_{x}=\overline{\mathcal{M}}_{\bar{x}}$.
} 
at $\bar{q}$. We have a commutative diagram

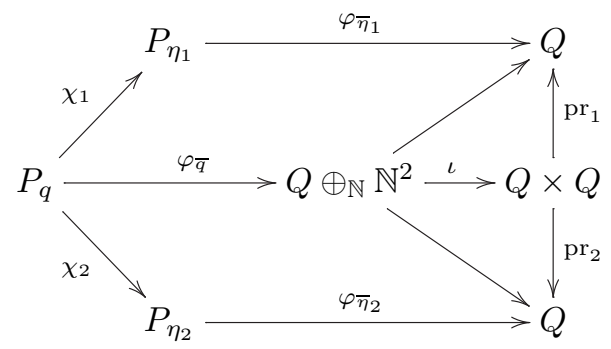

where the diagonal arrows are generization maps. Recall from (1.1) that $\iota$ is injective with $\left(m_{1}, m_{2}\right) \in Q \times Q$ in the image of $Q \oplus_{\mathbb{N}} \mathbb{N}^{2}$ iff $m_{1}-m_{2} \in \mathbb{Z} \rho_{q}$, viewed as an equation in $Q^{\mathrm{gp}}$. Thus $\varphi_{\bar{q}}$ is determined uniquely by $\varphi_{\bar{\eta}_{1}}, \varphi_{\bar{\eta}_{2}}$, and there exists a homomorphism

$$
u_{q}: P_{q} \longrightarrow \mathbb{Z}
$$

defined (since $\rho_{q} \neq 0$ ) by the equation

$$
\varphi_{\bar{\eta}_{2}}\left(\chi_{2}(m)\right)-\varphi_{\bar{\eta}_{1}}\left(\chi_{1}(m)\right)=u_{q}(m) \cdot \rho_{q} .
$$

Note that the definition of $u_{q}$ in (iii) depends on the choice of an ordering of the adjacent branches of $\underline{C}$. Such a choice can be implemented by orienting the dual intersection graph $\Gamma_{\underline{C}}$ of $\underline{C}$. The graph $\Gamma_{\underline{C}}$ has one vertex $v_{\eta}$ for each generic point $\eta \in \underline{C}$ and an edge $\bar{E}_{q}$ for each node $q$ joining $v_{\eta_{1}}$ and $v_{\eta_{2}}$ for $\eta_{1}, \eta_{2}$ the generic points of the two adjacent branches at $q$. Note that we can have $\eta_{1}=\eta_{2}$ if the component is not normal. In addition there is an unbounded edge (a flag) $E_{p}$ for each marked point $p$, with adjacent vertex $v_{\eta}$ for the unique generic point $\eta$ with $p \in \operatorname{cl}(\eta)$, the topological closure of $\eta$.

Remark 1.9. In the above discussion, we only needed to use the stalks $P_{x}$ of $\overline{\mathcal{M}}$ at points of $\underline{C}$ which were either generic, marked or double points. In fact, for any closed point $x \in \underline{C}$ which is neither a double point or marked point, we must have $P_{x}=P_{\eta}$, where $\eta$ is the generic point of the component of $C$ containing $x$. Indeed, we have a surjective generization map $\chi: P_{x} \rightarrow P_{\eta}$. Since $\overline{\mathcal{M}}$ is the ghost sheaf of a fine $\log$ structure, $\chi$ is given by localization at a face of $P_{x}$ followed by dividing out the submonoid of invertible elements. Thus, if $\chi$ is not an isomorphism, we can always find $0 \neq m \in P_{x}$ with $\chi(m)=0$. But $\overline{\mathcal{M}}_{C, \bar{x}}=\overline{\mathcal{M}}_{C, \bar{\eta}}=Q$, and since generization is compatible with the map $\varphi$ on stalks, we have $\varphi_{\bar{\eta}} \circ \chi=\varphi_{\bar{x}}$, hence $\varphi_{\bar{x}}(m)=0 \in Q$. This contradicts the property that $\varphi_{\bar{x}}$ is local.

Definition 1.10. 1) The type of an object $\left(Q, \overline{\mathcal{M}}_{C}, \psi, \varphi\right)$ of the category $\underline{\operatorname{GS}}(\overline{\mathcal{M}})$ is the set $\mathbf{u}:=\left\{u_{p} \in P_{p}^{\vee}, u_{q} \in P_{q}^{*}\right\}$, where $u_{p}$ and $u_{q}$ are defined in (1.6) and (1.7), respectively. Here $p$ and $q$ run over the marked and nodal points of $\underline{C}$, respectively. Given $\mathbf{u}$, the full subcategory of $\underline{\operatorname{GS}}(\overline{\mathcal{M}})$ with objects of type $\mathbf{u}$ is denoted $\underline{\operatorname{GS}}(\overline{\mathcal{M}}, \mathbf{u})$.

2) The type $\left(\Gamma_{\underline{C}}, \mathbf{u}\right)$ of a stable log map over a log point $(C /(\operatorname{Spec} \kappa, Q), \mathbf{x}, f)$ is the dual intersection graph $\Gamma_{\underline{C}}$ of $\underline{C}$ together with the type $\mathbf{u}$ of the corresponding object of $\underline{\operatorname{GS}}(\overline{\mathcal{M}})$. 
The type is compatible with generization:

Lemma 1.11. Let $\mathbf{u}, \mathbf{u}^{\prime}$ be the types of a stable log map $(C / W, \mathbf{x}, f)$ at two geometric points $\bar{w} \rightarrow \underline{W}, \bar{w}^{\prime} \rightarrow \underline{W}$ with $\bar{w} \in \operatorname{cl}\left(\bar{w}^{\prime}\right)$. For $x \in \underline{C}_{\bar{w}}, x^{\prime} \in \underline{C}_{\bar{w}^{\prime}}$ and $x \in \operatorname{cl}\left(x^{\prime}\right)$ let $\chi_{x^{\prime} x}: P_{x} \rightarrow P_{x^{\prime}}$ be the generization map of the stalks of $f^{*} \overline{\mathcal{M}}_{X}$. Then for marked or nodal points $x, x^{\prime}$ with $x \in \operatorname{cl}\left(x^{\prime}\right)$,

$$
u_{x}=u_{x^{\prime}} \circ \chi_{x^{\prime} x}
$$

Proof. For marked points $p, p^{\prime}$ this follows readily from compatibility of (1.6) with generization. For nodal points $q, q^{\prime}$ comparing the generization of (1.8) for $q$ with the equation for $q^{\prime}$ yields

$$
u_{q} \cdot \kappa\left(\rho_{q}\right)=\left(u_{q^{\prime}} \circ \chi_{q^{\prime} q}\right) \cdot \rho_{q^{\prime}},
$$

where $\kappa: \overline{\mathcal{M}}_{W, \bar{w}} \rightarrow \overline{\mathcal{M}}_{W, \bar{w}^{\prime}}$. The claimed equation now follows from $\kappa\left(\rho_{q}\right)=\rho_{q^{\prime}} \neq$ 0 .

1.4. The standard log point and tropical curves. An interesting special case is stable $\log$ maps over standard $\log$ points $(\operatorname{Spec} \kappa, \mathbb{N})$. This provides the connection to tropical geometry. In toric situations this connection has previously been discussed in NiSi] and in Gs, $\S 10$. Another motivation is that this case suffices to characterize universal stable log maps. To explain this we consider the situation of diagram (1.4) of a pre-stable curve $\underline{C} /$ Spec $\kappa$ and a fine saturated log structure $\mathcal{M}$ on $\underline{C}$. We think of the case $\overline{\mathcal{M}}=\underline{f}^{*} \mathcal{M}_{X}$ for an ordinary stable map $(\underline{C} / \operatorname{Spec} \kappa, \mathbf{x}, \underline{f})$. Now if diagram (1.4) is universal (for a fixed type) then diagrams of the same form over the standard log point are given by morphisms

$$
(\operatorname{Spec} \kappa, \mathbb{N}) \longrightarrow(\operatorname{Spec} \kappa, Q) \text {. }
$$

Moreover, two such morphisms lead to isomorphic log maps if and only if they differ by a homomorphism $Q \rightarrow \kappa^{\times}$. Now a morphism of $\log$ structures $Q \times \kappa^{\times} \longrightarrow \mathbb{N} \times \kappa^{\times}$ has the form

$$
(m, a) \longmapsto(\varphi(m), h(m) \cdot a)
$$

for some $\varphi \in \operatorname{Hom}(Q, \mathbb{N})$ with $\varphi^{-1}(0)=\{0\}$ and $h \in \operatorname{Hom}\left(Q, \kappa^{\times}\right)$. Composing with the automorphism

$$
(m, a) \longmapsto(m, h(m) \cdot a)
$$

of $($ Spec $\kappa, Q)$ we may assume $h=1$. Hence the set of isomorphism classes of stable log maps over $(\operatorname{Spec} \kappa, \mathbb{N})$ obtained from (1.4) by base change is in one-toone correspondence with

$$
\left\{\varphi \in \operatorname{Hom}(Q, \mathbb{N}) \mid \varphi^{-1}(0)=\{0\}\right\} .
$$

The upshot of this discussion is that $\operatorname{Int}\left(Q^{\vee}\right)$ is equal to the set of isomorphism classes of diagrams (1.4) with $Q=\mathbb{N}$. Note that while this is a discrete set, the set of isomorphism classes of diagrams (1.4) relative to a fixed $\log$ point $(\operatorname{Spec} \kappa, Q)$ is fibred over this discrete set with fibres $\operatorname{Hom}\left(Q, \kappa^{\times}\right)$.

Let us now specialize Discussion 1.8 to $Q=\mathbb{N}$. Then for a node $q$ the element $\rho_{q} \in Q \backslash\{0\}$ is a number $e_{q} \in \mathbb{N} \backslash\{0\}$, and $Q \oplus_{\mathbb{N}} \mathbb{N}^{2}$ is isomorphic to the submonoid $S_{e_{q}}$ of $\mathbb{N}^{2}$ generated by $\left(e_{q}, 0\right),\left(0, e_{q}\right),(1,1) 3$ Thus specifying the sheaf $\overline{\mathcal{M}}_{C}$ to-

\footnotetext{
${ }^{3}$ In terms of generators and relations we have $S_{e_{q}}=\left\langle a_{1}, a_{2}, a_{3} \mid a_{1}+a_{2}=e_{q} \cdot a_{3}\right\rangle$, where $a_{1}=\left(e_{q}, 0\right), a_{2}=\left(0, e_{q}\right), a_{3}=(1,1)$.
} 
gether with a homomorphism $\overline{\mathcal{M}} \rightarrow \overline{\mathcal{M}}_{C}$ is equivalent to the following data, for points $x \in \underline{C}$ :

(i) $x=\eta$ is a generic point. Then $\varphi_{\bar{\eta}}: P_{\eta} \longrightarrow \mathbb{N}$ defines an element $V_{\eta} \in P_{\eta}^{\vee}$.

(ii) $x=p$ is a marked point. As in the general case, $\varphi_{p}$ is determined by (i) and by $u_{p} \in P_{p}^{\vee}$, fixed by the type.

(iii) $x=q$ is a node and $\bar{\eta}_{1}, \bar{\eta}_{2}$ are the geometric generic points of the adjacent branches of $\underline{C}$. Letting $i_{q, \eta_{i}}: P_{\eta_{i}}^{\vee} \rightarrow P_{q}^{\vee}$ be the inclusion induced by the generization maps, (1.8) now reads

$$
i_{q, \eta_{2}}\left(V_{\eta_{2}}\right)-i_{q, \eta_{1}}\left(V_{\eta_{1}}\right)=e_{q} u_{q} .
$$

Thus apart from the type $\mathbf{u}$ a stable log map over a standard log point defines points $V_{\eta} \in P_{\eta}^{\vee}$ and $e_{q} \in \mathbb{N} \backslash\{0\}$. We call the tuple $\left(\left(V_{\eta}\right)_{\eta},\left(e_{q}\right)_{q}\right)$ tropical data of a stable log map over a standard log point. Similarly, we can talk of tropical data for an object $\left(Q, \overline{\mathcal{M}}_{C}, \psi, \varphi\right)$ of $\underline{\operatorname{GS}}(\overline{\mathcal{M}})$ with $Q=\mathbb{N}$.

To discuss the relationship with tropical geometry we recall the basic definition of a tropical curve. By abuse of notation we confuse a graph and its topological realization.

Definition 1.12. Let $\bar{\Gamma}$ be a connected graph and let $\Gamma$ be the topological space obtained by removing from $\bar{\Gamma}$ a set of univalent vertices of $\bar{\Gamma}$, so that $\Gamma$ has both compact and non-compact edges 4 We assume that $\Gamma$ has at least one vertex. Let $N \simeq \mathbb{Z}^{n}$ be a lattice, $N_{\mathbb{R}}:=N \otimes_{\mathbb{Z}} \mathbb{R}$. A tropical curve in $N_{\mathbb{R}}$ with domain $\Gamma$ consists of the following data:

(i) For each flag $(v, E)$ of $\Gamma$, where $v$ is a vertex of $\Gamma$ and $E$ an edge containing $v$, we are given a weight vector $u_{(v, E)} \in N$. If $E$ has two vertices $v_{1}$ and $v_{2}$, then $u_{\left(v_{1}, E\right)}=-u_{\left(v_{2}, E\right)}$, and if $E$ is a loop, then $u_{(v, E)}=0$. The graph $\Gamma$ along with the weight vectors is called the type of the tropical curve.

(ii) A map $h: \Gamma \rightarrow N_{\mathbb{R}}$ with the following properties:

(a) For any edge $E$ of $\Gamma$ with vertex $v,\left.h\right|_{E}$ is constant if $u_{(v, E)}=0$, and otherwise $\left.h\right|_{E}$ is proper and identifies $E$ with an affine line segment or ray. Furthermore, $u_{(v, E)}$ is a tangent vector to $h(E)$ pointing away from $h(v)$.

(b) For each vertex $v$, we have the balancing condition

$$
\sum_{E} u_{(v, E)}=0
$$

where the sum is over all edges $E$ with vertex $v$.

Discussion 1.13. The term "tropical data" is motivated by the case that $\overline{\mathcal{M}}^{\mathrm{gp}}$ is globally generated. In this case the tropical data gives rise to a generalized tropical curve with domain $\left|\Gamma_{\underline{C}}\right|$, the geometric realization of $\Gamma_{\underline{C}}$, and image in $N_{\mathbb{R}}$ for

$$
N:=\operatorname{Hom}\left(\Gamma\left(\underline{C}, \overline{\mathcal{M}}^{\mathrm{gp}}\right), \mathbb{Z}\right),
$$

as follows. The generalization concerns the balancing condition, as to be discussed. The restriction maps $\Gamma\left(\underline{C}, \overline{\mathcal{M}}^{\mathrm{gp}}\right) \rightarrow \overline{\mathcal{M}}_{\bar{x}}^{\mathrm{gp}}$ induce injections

$$
P_{x}^{\vee} \longrightarrow N \text {. }
$$

\footnotetext{
${ }^{4}$ The set of univalent vertices to be removed is part of the data. A traditional tropical curve in $\mathbb{R}^{n}$ is the image of $\Gamma$, and $\bar{\Gamma}$ is obtained by attaching a univalent vertex at each unbounded edge.
} 
Denote by $\tilde{V}_{\eta}, \tilde{u}_{p}, \tilde{u}_{q}$ the images of $V_{\eta}, u_{p}, u_{q}$ in $N$. Define a continuous map

$$
h:\left|\Gamma_{\underline{C}}\right| \longrightarrow N_{\mathbb{R}}
$$

by sending $v_{\eta}$ to $\tilde{V}_{\eta}$, an edge $E_{q}$ with adjacent vertices $v_{\eta_{1}}, v_{\eta_{2}}$ to the line segment connecting $\tilde{V}_{\eta_{1}}$ and $\tilde{V}_{\eta_{2}}$, and an unbounded edge $E_{p}$ with adjacent vertex $v_{\eta}$ to the ray $\tilde{V}_{\eta}+\mathbb{R}_{\geq 0} \tilde{u}_{p}$. The weight vectors are given by $u_{\left(v_{\eta}, E_{p}\right)}:=\tilde{u}_{p}$ for the unbounded edges and $u_{\left(v_{\eta_{i}}, E_{q}\right)}:= \pm \tilde{u}_{q}$, with the sign chosen so that $u_{\left(v_{\eta_{i}}, E_{q}\right)}$ points away from $\tilde{V}_{\eta_{i}}$. Note that by (1.9) for an edge $E_{q}$ with vertices $v_{\eta_{1}}, v_{\eta_{2}}, h\left(v_{\eta_{2}}\right)-h\left(v_{\eta_{1}}\right)=$ $\pm e_{q} \tilde{u}_{q}$, so $e_{q}$ is the integral length of the corresponding edge of the tropical curve, as a multiple of the weight vector, at least for $\tilde{u}_{q} \neq 0$.

As is, this does not in general fulfill the balancing condition (Definition 1.12(b)). However, in Proposition [1.15] below we formulate a modified balancing condition involving a correction term. The correction term turns out to depend only on the given $\log$ structure $\mathcal{M}$ on $\underline{C}$, that is, on the underlying ordinary stable map. The balancing condition holds unmodified if for any irreducible component $D \subset \underline{C}$ and $m \in \Gamma\left(D,\left.\overline{\mathcal{M}}\right|_{D}\right)$ the degree of the corresponding $\mathcal{O}_{D}^{\times}$-torsor $\left.L_{m} \subset \mathcal{M}\right|_{D}$ vanishes 5 . In general one can add one more unbounded edge at each vertex $v_{\eta}$ with the weight vector $\tau_{\eta}^{X}$ derived from $\underline{f}$ via $(1.10)$ to obtain an honest tropical curve in $\mathrm{N}_{\mathbb{R}}$.

In the toric degenerations of toric varieties of [NiSi] already $\overline{\mathcal{M}}_{X}^{\text {gp }}$ is globally generated. It is then appropriate to consider the composition with

$$
N \longrightarrow N^{\prime}:=\operatorname{Hom}\left(\Gamma\left(\underline{X}, \overline{\mathcal{M}}_{X}^{\mathrm{gp}}\right), \mathbb{Z}\right) .
$$

Moreover, since in $\mathrm{NiSi}$ ] we work relative $(\operatorname{Spec} \mathbb{k}, \mathbb{N})$ the images of the generator of $\overline{\mathcal{M}}_{\text {Spec } \mathbb{k}}=\mathbb{N}$ under $X \rightarrow(\operatorname{Spec} \mathbb{k}, \mathbb{N})$ and under $(\operatorname{Spec} \kappa, \mathbb{N}) \rightarrow(\operatorname{Spec} \mathbb{k}, \mathbb{N})$ define a global section $\rho$ of $\overline{\mathcal{M}}_{X}$ and an element $b \in \mathbb{N}=\overline{\mathcal{M}}_{(\text {Spec } \kappa, \mathbb{N})}$, respectively. Commutativity of (1.3) at a generic point $\eta$ now implies $V_{\eta}\left(\rho_{f(\eta)}\right)=b$. Thus $h$ maps the vertices of $\Gamma$ to the affine hyperplane $H \subset N_{\mathbb{R}}^{\prime}$ defined by $\langle\rho,\rangle=$.$b . Finally, from$ the structure of $\log$ smooth curves at a marked point, we have $\left\langle\rho, \tilde{u}_{p}\right\rangle=u_{p}\left(\rho_{p}\right)=0$, and hence $\operatorname{im}(h) \subset H$. It is the map to $H$ that traditionally and in NiSi is called a tropical curve. This ends our discussion of tropical curves in this context.

As suggested by the tropical curve interpretation of Discussion 1.13 there should be a balancing condition at each vertex $v_{\eta}$ of $\Gamma_{\underline{C}}$ imposing restrictions on $u_{p}, u_{q}$ for the adjacent edges $E_{p}, E_{q}$. Denote $\mathcal{M}:=\underline{f}^{*} \overline{\mathcal{M}}_{X}$. For a generic point $\eta \in \underline{C}$ let $D:=\operatorname{cl}(\eta)$ and let $g: \tilde{D} \rightarrow \underline{C}$ be the normalization of $D$. This gives rise to maps which are compositions:

$$
\begin{aligned}
& \tau_{\eta}^{X}: \quad \Gamma\left(\tilde{D}, g^{*} \overline{\mathcal{M}}\right) \longrightarrow \operatorname{Pic} \tilde{D} \stackrel{\text { deg }}{\longrightarrow} \mathbb{Z}, \\
& \tau_{\eta}^{C}: \quad \Gamma\left(\tilde{D}, g^{*} \overline{\mathcal{M}}_{C}\right) \longrightarrow \operatorname{Pic} \tilde{D} \stackrel{\operatorname{deg}}{\longrightarrow} \mathbb{Z} .
\end{aligned}
$$

The first map associates to a section of $g^{*} \overline{\mathcal{M}}$ or $g^{*} \overline{\mathcal{M}}_{C}$ the corresponding $\mathcal{O}_{D}^{\times}$-torsor, and the second map is the degree homomorphism.

The balancing condition is due to the basic fact that $f^{b}$ must induce isomorphisms of torsors, so that the pull-back $\varphi: g^{*} \overline{\mathcal{M}} \rightarrow g^{*} \overline{\mathcal{M}}_{C}$ of $\overline{f^{b}}$ to $\tilde{D}$ fits into the

\footnotetext{
${ }^{5}$ Recall that if $\mathcal{M}$ is a log structure on a space $X$, then any section $m \in \Gamma(X, \overline{\mathcal{M}})$ gives rise to an $\mathcal{O}_{X}^{\times}$torsor $\kappa^{-1}(m) \subset \mathcal{M}$ for $\kappa: \mathcal{M} \rightarrow \overline{\mathcal{M}}$ the quotient homomorphism.
} 
commutative diagram

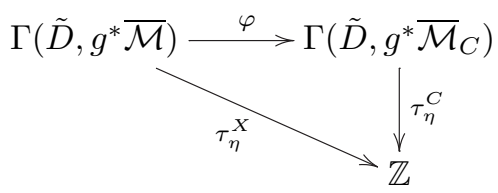

The map $\tau_{\eta}^{X}$ is given by $f$ and $\mathcal{M}$, so we have no control over it, except that if $\underline{f}$ contracts $D$, then $\tau_{\eta}^{X}=0$. Otherwise there is nothing general we can say.

Similarly, $\tau_{\eta}^{C}$ is determined by $\mathcal{M}_{C}$. Explicitly, for $q \in D$ identify $S_{e_{q}} \subset \mathbb{N}^{2}$ with the submonoid generated by $\left(0, e_{q}\right),\left(e_{q}, 0\right),(1,1)$ so that the generization map $\chi_{q}: \overline{\mathcal{M}}_{C, \bar{q}}=S_{e_{q}} \rightarrow \overline{\mathcal{M}}_{D, \bar{\eta}}=\mathbb{N}$ is the projection onto the second coordinate: $\chi_{q}(a, b)=b$. We have the identification

$$
\Gamma\left(\tilde{D}, g^{*} \overline{\mathcal{M}}_{C}\right)=\left\{\left(n_{q}\right)_{q \in \tilde{D}} \mid n_{q} \in S_{e_{q}} \text { and } \chi_{q}\left(n_{q}\right)=\chi_{q^{\prime}}\left(n_{q^{\prime}}\right) \text { for } q, q^{\prime} \in \tilde{D}\right\} \oplus \bigoplus_{p \in \tilde{D}} \mathbb{N} \text {. }
$$

In particular, if $\left(\left(a_{q}, b_{q}\right),\left(n_{p}\right)\right)$ represents an element of $\Gamma\left(\tilde{D}, g^{*} \overline{\mathcal{M}}_{C}\right)$, then all second entries $b_{q}$ agree.

Lemma 1.14. $\tau_{\eta}^{C}\left(\left(\left(a_{q}, b\right)_{q \in \tilde{D}},\left(n_{p}\right)_{p \in \tilde{D}}\right)\right)=-\sum_{p \in \tilde{D}} n_{p}+\sum_{q \in \tilde{D}} \frac{b-a_{q}}{e_{q}}$.

Proof. By log smoothness the element $\left((b, b)_{q},(0)_{p}\right) \in \Gamma\left(\tilde{D}, g^{*} \overline{\mathcal{M}}_{C}\right)$ with all $a_{q}=b$, $n_{p}=0$, maps to the trivial $\mathcal{O}_{\tilde{D}}^{\times}$-torsor. Thus it suffices to consider sections of $g^{*} \overline{\mathcal{M}}_{C}$ of the form $\left(\left(a_{q}, 0\right),\left(n_{p}\right)\right)$ with $a_{q} / e_{q} \in \mathbb{Z}$. Let $L \subset g^{*} \mathcal{M}_{C}$ be the corresponding $\mathcal{O}_{\tilde{D}}^{\times}$-torsor. The structure map $L \rightarrow g^{*} \mathcal{M}_{C} \rightarrow \mathcal{O}_{\tilde{D}}$ identifies $L$ with the sheaf of regular functions on $\tilde{D}$ with zeros of order $a_{q} / e_{q}$ at $q$ and of order $n_{p}$ at $p$. In fact, if the $\log$ structure at $q$ is induced from the toric model Spec $\kappa[x, y, t] /\left(x y-t^{e_{q}}\right)$ with $\tilde{D}$ corresponding to $V(y)$, then $x$ defines an element of $\mathcal{M}_{C, \bar{q}}$ mapping to $\left(e_{q}, 0\right) \in S_{e_{q}}$. Hence

$$
\operatorname{deg}(L)=\operatorname{deg} \mathcal{O}_{\tilde{D}}\left(-\sum_{q} \frac{a_{q}}{e_{q}} q-\sum_{p} n_{p} p\right)=-\sum_{q} \frac{a_{q}}{e_{q}}-\sum_{p} n_{p} .
$$

This is the claimed formula.

The equation $\tau_{\eta}^{X}=\tau_{\eta}^{C} \circ \varphi$ is a formula in $N_{D}:=\Gamma\left(\tilde{D}, g^{*} \overline{\mathcal{M}}^{\mathrm{gp}}\right)^{*}$, which is the inductive limit of abelian groups $\left(P_{x}^{\vee}\right)^{\mathrm{gp}}=\left(P_{x}^{\mathrm{gp}}\right)^{*}$ with respect to the homomorphisms $\iota_{x, \eta}:\left(P_{\eta}^{\vee}\right)^{\mathrm{gp}} \rightarrow\left(P_{x}^{\vee}\right)^{\mathrm{gp}}, x \in \tilde{D}$. Here $P_{x}$ for $x \in \tilde{D}$ means $P_{g(x)}$. More explicitly, if $\Sigma \subset \tilde{D}$ is the set of special points $p, q$, that is, mapping to a special point in $D$, then

$$
N_{D}=\underset{x \in \tilde{D}}{\lim _{x}}\left(P_{x}^{\vee}\right)^{\mathrm{gp}}=\left(\bigoplus_{x \in \Sigma}\left(P_{x}^{\vee}\right)^{\mathrm{gp}}\right) / \sim,
$$

where for any $a \in\left(P_{\eta}^{\vee}\right)^{g p}$ and $x, x^{\prime} \in \Sigma$,

$$
\left(0, \ldots, 0, \iota_{x, \eta}(a), 0, \ldots, 0\right) \sim\left(0, \ldots, 0, \iota_{x^{\prime}, \eta}(a), 0, \ldots, 0\right) .
$$

We may thus represent an element of $N_{D}$ as a tuple $\left(a_{x}\right)_{x \in \Sigma}$, but keep in mind the relations (1.13).

With this representation of $N_{D}$ we are now in position to write down the balancing condition. 
Proposition 1.15. Consider the diagram (1.4) with $Q=\mathbb{N}$ and $D \subset \underline{C}$ an irreducible component with generic point $\eta$ and $\Sigma \subset \tilde{D}$ the preimage of the set of special points. If $\tau_{\eta}^{X}$ defined in (1.10) is represented by $\left(\tau_{x}\right)_{x \in \Sigma}$, then

$$
\left(u_{x}\right)_{x \in \Sigma}+\left(\tau_{x}\right)_{x \in \Sigma}=0
$$

in $N_{D}=\Gamma\left(\tilde{D}, \overline{\mathcal{M}}^{\mathrm{gp}}\right)^{*}$.

Proof. Let $m \in \Gamma\left(\tilde{D}, g^{*} \overline{\mathcal{M}}\right)$. Recall that $\varphi$ denoted the pull-back of $\overline{f^{b}}$ by $g$. In view of Lemma 1.14,

$$
\begin{aligned}
\tau_{\eta}^{C}(\varphi(m)) & =\tau_{\eta}^{C}\left(\left(\left\langle V_{\eta_{q}}, m\right\rangle,\left\langle V_{\eta}, m\right\rangle\right)_{q \in \tilde{D}},\left(\left\langle u_{p}, m\right\rangle\right)_{p \in D}\right) \\
& =\sum_{q \in \tilde{D}} \frac{1}{e_{q}}\left(\left\langle V_{\eta}, m\right\rangle-\left\langle V_{\eta_{q}}, m\right\rangle\right)-\sum_{p \in \tilde{D}}\left\langle u_{p}, m\right\rangle .
\end{aligned}
$$

Thus, since $u_{q}(m)=\frac{1}{e_{q}}\left(\left\langle V_{\eta_{q}}, m\right\rangle-\left\langle V_{\eta}, m\right\rangle\right)$,

$$
\tau_{\eta}^{C} \circ \varphi=\left(\left(-u_{q}\right)_{q \in \tilde{D}},\left(-u_{p}\right)_{p \in \tilde{D}}\right)
$$

and the claimed formula follows from $\tau_{\eta}^{C} \circ \varphi=\tau_{\eta}^{X}$, the commutativity of (1.11).

1.5. The basicness condition. For a fine saturated sheaf $\overline{\mathcal{M}}$ over a pre-stable curve over a field $\underline{C} / \operatorname{Spec} \kappa$, in Definition 1.7 we introduced the category $\underline{\operatorname{GS}}(\overline{\mathcal{M}})$. Given a type $\mathbf{u}$ for objects of $\mathrm{GS}(\overline{\mathcal{M}})$ (Definition 1.10) we are now in position to construct a universal object for the full subcategory $\underline{\operatorname{GS}}(\overline{\mathcal{M}}, \mathbf{u})$.

Construction 1.16. Let $\mathbf{u}=\left\{\left(u_{p}\right)_{p},\left(u_{q}\right)_{q}\right\}$ be a type for $\underline{\operatorname{GS}}(\overline{\mathcal{M}})$ and assume $\underline{\mathrm{GS}}(\overline{\mathcal{M}}) \neq \emptyset$. For a node $q \in \underline{C}$ denote by $\chi_{\eta_{i}, q}: P_{q} \rightarrow P_{\eta_{i}}$ the two generization maps, ordered as in the definition of $u_{q}$ in (1.8). Then if $m \in P_{q}$, let

$a_{q}(m):=\left(\left(\ldots, \chi_{\eta_{1}, q}(m), \ldots,-\chi_{\eta_{2}, q}(m), \ldots\right),\left(\ldots, u_{q}(m), \ldots\right)\right) \in\left(\prod_{\eta} P_{\eta} \times \prod_{q} \mathbb{N}\right)^{\mathrm{gp}}$

be the element with all entries vanishing except the indicated ones at places $\eta_{1}, \eta_{2}$ and $q$. Let $R \subset\left(\prod_{\eta} P_{\eta} \times \prod_{q} \mathbb{N}\right)^{\text {gp }}$ be the saturated subgroup generated by the $a_{q}(m)$ for all nodes $q \in \underline{C}$ and $m \in P_{q}$. Now define the basic monoid $Q$ as the saturation of the quotient by $R$ :

$$
Q:=\left[\iota\left(\prod_{\eta \in \underline{C}} P_{\eta} \times \prod_{q \in \underline{C}} \mathbb{N}\right) / R\right]^{\text {sat }} .
$$

Here $\iota$ denotes the inclusion of $\prod_{\eta} P_{\eta} \times \prod_{q} \mathbb{N}$ into its associated group. By the very definition $Q$ is fine and saturated. Taking the saturation of $R$ amounts to dividing out any torsion of the associated group, so $Q$ is also torsion-free. But note that at this point there is no reason to infer $Q^{\times}=\{0\}$, and in fact, this is not true in general.

The inclusion of the various factors into $\prod_{\eta} P_{\eta} \times \prod_{q} \mathbb{N}$ composed with the surjection to $Q$ defines the homomorphisms

$$
\begin{aligned}
& \varphi_{\bar{\eta}}: P_{\eta} \longrightarrow \prod_{\eta} P_{\eta} \times \prod_{q} \mathbb{N} \longrightarrow Q, \\
& \mathbb{N} \longrightarrow \prod_{\eta} P_{\eta} \times \prod_{q} \mathbb{N} \longrightarrow Q, \quad 1 \longmapsto \rho_{q} .
\end{aligned}
$$


Since the difference of the two sides of Equation (1.8) is nothing but $a_{q}(m)$, we have the following equality of maps $P_{q} \rightarrow Q$ (where $\chi_{i}=\chi_{\eta_{i}, q}$ ):

$$
\varphi_{\bar{\eta}_{2}} \circ \chi_{2}-\varphi_{\bar{\eta}_{1}} \circ \chi_{1}=u_{q} \cdot \rho_{q} .
$$

From Discussion 1.8, (i)-(iii) the data $Q, \rho_{q}$ and $\varphi_{\bar{\eta}}$ thus define a distinguished object $\left(Q, \overline{\mathcal{M}}_{C}, \psi, \varphi\right)$ of $\underline{\operatorname{GS}}(\overline{\mathcal{M}}, \mathbf{u})$, except that we do not know at this point that $Q^{\times}=0$ and that all morphisms are local. These properties will be established in Proposition 1.19 provided $\underline{\mathrm{GS}}(\overline{\mathcal{M}}, \mathbf{u}) \neq \emptyset$.

Example 1.17. Let us illustrate some features of this definition with some simple examples, notably concerning the locality property of homomorphisms and saturation issues.

(1) Consider a curve $\underline{C}$ with two irreducible components mutually intersecting in two nodes $q_{1}, q_{2}$. Assume that the whole curve maps to a standard log point, and hence $P_{x}=\mathbb{N}$ for all $x$ and all generization maps are isomorphisms. Choose $u_{q_{1}}(1)=0, u_{q_{2}}(1)=1$. Then in $\prod_{\eta} P_{\eta}^{\mathrm{gp}} \times \prod_{q} \mathbb{Z}=\mathbb{Z}^{4}$ we have

$$
a_{q_{1}}(1)=(1,-1,0,0), \quad a_{q_{2}}(1)=(1,-1,0,1) .
$$

Thus $(0,0,0,1) \in R$ and hence $\rho_{q_{2}}=0$. Thus at $q_{2}$ the morphism of monoids $\mathbb{N} \rightarrow \overline{\mathcal{M}}_{\underline{C}, q_{2}}$ mapping 1 to $\rho_{q}$ is not local. In particular, $\left(\left(\underline{C}, \overline{\mathcal{M}}_{C}\right) /\right.$ $(\operatorname{Spec} \kappa, \mathbb{N}), \mathbf{x})$ is not a pre-stable log curve on the level of ghost sheaves (Definition 1.5).

For a geometric interpretation of the situation note that $u_{q}$ compares the lifts of elements of the $\log$ structure of $X$ to the two branches of $\underline{C}$ at $q$. In the present situation the result at the two nodes has to agree since $\underline{C}$ has only two irreducible components. This can be viewed as a manifestation in log geometry of the impossibility of a deformation situation where one of the two nodes smooths while the other stays.

(2) Considering the same situation as in (1) but with $u_{q_{1}}(1)=2, u_{q_{2}}(1)=3$, leads to a non-saturated image of $\prod_{\eta} P_{\eta} \times \prod_{q} \mathbb{N}$. In fact, $R$ is now generated by

$$
a_{q_{1}}(1)=(1,-1,2,0), \quad a_{q_{2}}(1)=(1,-1,0,3),
$$

and the map

$$
\left(\begin{array}{cccc}
1 & 1 & 0 & 0 \\
-6 & 0 & 3 & 2
\end{array}\right): \mathbb{Z}^{4} \longrightarrow \mathbb{Z}^{2}
$$

describes the quotient of $\prod_{\eta} P_{\eta}^{\mathrm{gp}} \times \prod_{q} \mathbb{Z}=\mathbb{Z}^{4}$ by $R$. The image of $\prod_{\eta} P_{\eta} \times$ $\prod_{q} \mathbb{N}=\mathbb{N}^{4}$ is then generated by the columns of the matrix, and hence is not saturated, for it contains $(0,2)$ and $(0,3)$ but not $(0,1)$. Thus we need to saturate in the definition of $Q$ to stay in the category of fine saturated $\log$ schemes 6

(3) Consider again a curve as in (1), but now mapping to a pre-stable log curve with one node in such a way that

$$
P_{q_{1}}=\mathbb{N}^{2}, \quad P_{\eta_{1}}=P_{q_{2}}=P_{\eta_{2}}=\mathbb{N},
$$

with only non-trivial generization maps $P_{q_{1}} \rightarrow P_{\eta_{i}}$ the two projections $\mathbb{N}^{2} \rightarrow \mathbb{N}$. Take $u_{q_{1}}(a, b)=a+b, u_{q_{2}}(c)=2 c$. Then

$$
a_{q_{1}}(1,0)=(1,0,1,0), \quad a_{q_{1}}(0,1)=(0,-1,1,0), \quad a_{q_{2}}(1)=(1,-1,0,2) .
$$


The subgroup of $\prod_{\eta} P_{\eta}^{\text {gp }} \times \prod_{q} \mathbb{Z}=\mathbb{Z}^{4}$ generated by these elements contains $(0,0,2,-2)=a_{q_{1}}(1,1)-a_{q_{2}}(1)$, but it does not contain $(0,0,1,-1)$. Hence saturation in the definition of $R$ is necessary to make $\left(\prod_{\eta} P_{\eta}^{\mathrm{gp}} \times \prod_{q} \mathbb{Z}\right) / R$ torsion-free.

Remark 1.18. Another useful way of thinking about $Q$ is in the dual space:

$$
Q^{\vee}=\left\{\left(\left(V_{\eta}\right)_{\eta},\left(e_{q}\right)_{q}\right) \in \bigoplus_{\eta} P_{\eta}^{\vee} \oplus \bigoplus_{q} \mathbb{N} \mid \forall q: V_{\eta_{2}}-V_{\eta_{1}}=e_{q} u_{q}\right\} .
$$

In particular, this avoids any saturation issues. Note that following the discussion in $\$ 1.4$ any $\left(\left(V_{\eta}\right),\left(e_{q}\right)\right) \in Q^{\vee}$ that does not lie in any proper face gives rise to an object in the category $\underline{\operatorname{GS}}(\overline{\mathcal{M}})$ of the form $\left(\mathbb{N}, \overline{\mathcal{M}}_{C}, \psi, \phi\right)$. In fact, this is how the authors first found $Q$.

Proposition 1.19. If $\underline{\operatorname{GS}}(\overline{\mathcal{M}}, \mathbf{u}) \neq \emptyset$, then it has as initial object the tuple $\left(Q, \overline{\mathcal{M}}_{C}, \psi, \varphi\right)$ from Construction 1.16 .

Proof. We first prove the universal property in an enlarged category without the assumption that $Q^{\times}=\{0\}$ and the morphisms of monoids local. Let $\left(Q^{\prime}, \overline{\mathcal{M}}_{C}^{\prime}, \psi^{\prime}, \varphi^{\prime}\right)$ $\in \mathrm{Ob}(\underline{\mathrm{GSS}}(\overline{\mathcal{M}}, \mathbf{u}))$. Let $\rho_{q}^{\prime} \in Q^{\prime}$ be the element defining $\overline{\mathcal{M}}_{C, \bar{q}}^{\prime}$. Then because of (1.8) for $\left(Q^{\prime}, \overline{\mathcal{M}}_{C}^{\prime}, \psi^{\prime}, \varphi^{\prime}\right)$ and since $Q^{\prime}$ is torsion-free and saturated the map

$$
\prod_{\eta} P_{\eta} \times \prod_{q} \mathbb{N} \stackrel{\prod_{\eta} \varphi_{\bar{\eta}}^{\prime} \times \prod_{q} \rho_{q}^{\prime}}{\longrightarrow} Q^{\prime}
$$

factors over $Q$. Tracing the image of the generator of the $q$-th copy of $\mathbb{N}$ shows that the induced map $Q \rightarrow Q^{\prime}$ maps $\rho_{q}$ to $\rho_{q}^{\prime}$. Moreover, by the very definition of this factorization it is compatible with $\varphi_{\bar{\eta}}: P_{\eta} \rightarrow Q$ and $\varphi_{\bar{\eta}}^{\prime}: P_{\eta} \rightarrow Q^{\prime}$. This proves existence of a morphism $\left(Q, \overline{\mathcal{M}}_{C}, \psi, \varphi\right) \rightarrow\left(Q^{\prime}, \overline{\mathcal{M}}_{C}^{\prime}, \psi^{\prime}, \varphi^{\prime}\right)$.

For uniqueness note that by compatibility of $\rho_{q}, \rho_{q}^{\prime}$ and of $\varphi_{\bar{\eta}}, \varphi_{\bar{\eta}}^{\prime}$ any such morphism would lift to the homomorphism stated in (1.15).

It remains to show that $\left(Q, \overline{\mathcal{M}}_{C}, \psi, \varphi\right) \in \underline{\mathrm{GS}}(\overline{\mathcal{M}}, \mathbf{u})$, that is, that $Q^{\times}=0$ and all sheaf homomorphisms are local. Since $\underline{\mathrm{GS}}(\overline{\mathcal{M}}, \mathbf{u}) \neq \emptyset$ there is at least one morphism $\left(Q, \overline{\mathcal{M}}_{C}, \psi, \varphi\right) \rightarrow\left(Q^{\prime}, \overline{\mathcal{M}}_{C}^{\prime}, \psi^{\prime}, \varphi^{\prime}\right)$ as constructed above. Now because $\left(Q^{\prime}, \overline{\mathcal{M}}_{C}^{\prime}, \psi^{\prime}, \varphi^{\prime}\right) \in \mathrm{Ob}(\underline{\mathrm{GS}}(\overline{\mathcal{M}}, \mathbf{u}))$, for any $\eta$ and $q$ the compositions

$$
P_{\eta} \stackrel{\varphi_{\bar{\eta}}}{\longrightarrow} Q \longrightarrow Q^{\prime}, \quad \mathbb{N} \stackrel{\cdot \rho_{q}}{\longrightarrow} Q \longrightarrow Q^{\prime}
$$

are local. This proves that $\psi$ and $\varphi$ are indeed local homomorphisms. Thus also the composition

$$
\prod_{\eta} P_{\eta} \times \prod_{q} \mathbb{N} \longrightarrow Q \longrightarrow Q^{\prime}
$$

is local and hence, by surjectivity of the first arrow up to saturation, also $Q \rightarrow$ $Q^{\prime}$ is local and $Q^{\times}=\{0\}$. Finally, $\overline{\mathcal{M}}_{C} \rightarrow \overline{\mathcal{M}}_{C}^{\prime}$ is local because at a node the homomorphism is defined by the product of the homomorphisms at the adjacent generic points.

Definition 1.20. A stable log map $(C / W, \mathbf{x}, f)$ is called basic if for any geometric point $\bar{w} \rightarrow \underline{W}$ the induced object of $\underline{\mathrm{GS}}\left(\underline{f}_{\bar{w}}^{*} \overline{\mathcal{M}}_{X}, \mathbf{u}\right)$ is universal. Here $\mathbf{u}$ is the type of $(C / W, \mathbf{x}, f)$ at $\bar{w}$. 
Remark 1.21. Following up on Remark[1.18 the tropical interpretation of the basicness condition is as follows. If $\bar{w}: \operatorname{Spec} \kappa \rightarrow \underline{W}$ is a geometric point, then $\operatorname{Int} \overline{\mathcal{M}}_{W, \bar{w}}^{\vee}$ can be identified with the set of tropical data of the pull-backs to $(\operatorname{Spec} \kappa, \mathbb{N})$ via a $\log$ enhancement of $\bar{w}$. Moreover, in the proper tropical situations of Discussion 1.13 the real cone generated by $\overline{\mathcal{M}}_{W, \bar{w}}^{\vee}$ is canonically isomorphic to

$\operatorname{Hom}\left(Q, \mathbb{R}_{\geq 0}\right)=\left\{\left(\left(V_{\eta}\right)_{\eta},\left(l_{q}\right)_{q}\right) \in \bigoplus_{\eta} \operatorname{Hom}\left(P_{\eta}, \mathbb{R}_{\geq 0}\right) \oplus \bigoplus_{q} \mathbb{R}_{\geq 0} \mid \forall q: V_{\eta_{2}}-V_{\eta_{1}}=l_{q} u_{q}\right\}$.

Each point in this space defines a tropical curve with vertices $V_{\eta}$ and interior edges $q$ mapping to a translation of the line segment connecting 0 with $l_{q} u_{q}$. This can be interpreted as the moduli space of tropical curves of the given type. The proper faces of $\operatorname{Hom}\left(Q, \mathbb{R}_{\geq 0}\right)$ parametrize degenerate curves for which there may be some edge of length 0 or some vertex $V_{\eta}$ which maps to the boundary of $\operatorname{Hom}\left(P_{\eta}, \mathbb{R}_{\geq 0}\right)$.

As a first property we show that basicness is an open condition:

Proposition 1.22. Let $(C / W, \mathbf{x}, f)$ be a stable log map to a log scheme $X$. Then

$$
\Omega:=\left\{\bar{w} \in|\underline{W}| \mid\{\bar{w}\} \times_{\underline{W}}(C / W, \mathbf{x}, f) \text { is basic }\right\}
$$

is an open subset of $|\underline{W}| \mathbf{7}$

Proof. Since basicness is a condition on morphisms of fine sheaves, $\Omega$ is constructible 8 It remains to show that $\Omega$ is closed under generization. So let $\bar{w}_{1} \in \Omega$, $\bar{w}_{2} \in|\underline{W}|$ and $\bar{w}_{1} \in \operatorname{cl}\left(\bar{w}_{2}\right)$. We need to show $\bar{w}_{2} \in \Omega$. Since basicness is stable under strict base change we may first replace $\underline{W}$ by $\operatorname{Spec}\left(\mathcal{O}_{\underline{W}}, \bar{w}_{1}\right)$ and then by $\operatorname{cl}\left(\bar{w}_{2}\right)$ with the induced reduced scheme structure, to reduce to the case $\underline{W}=\operatorname{Spec} R$ for a strictly Henselian local domain $R$, and with $\bar{w}_{1}$ and $\bar{w}_{2}$ the closed point 0 and generic point Spec $K, K$ the quotient field of $R$. Denote by $\kappa=R / \mathfrak{m}$ the residue field of $R$, and endow $\operatorname{Spec} \kappa$ and $\operatorname{Spec} K$ with the $\log$ structures induced by the embeddings into $W=\left(\operatorname{Spec} R, \mathcal{M}_{R}\right)$.

Now we have two relevant stable log maps over fields, the closed fibre

$$
\left(C_{0} /(\operatorname{Spec} \kappa, Q), \mathbf{x}_{0}, f_{0}\right):=\operatorname{Spec} \kappa \times_{\underline{W}}(C / W, \mathbf{x}, f),
$$

which is basic by assumption, and the generic fibre

$$
\left(C_{K} /\left(\operatorname{Spec} K, Q_{K}\right), \mathbf{x}_{K}, f_{K}\right):=\operatorname{Spec} K \times_{\underline{W}}(C / W, \mathbf{x}, f),
$$

a stable log map over some log point $\left(\operatorname{Spec} K, Q_{K}\right)$. We use the standard notations for the points and the monoids of the closed fibre, while for the generic fibre we add hats. Note that apart from the usual generization maps between points on the same fibre we also have generization homomorphisms from the closed to the generic fibre,

$$
P_{\eta} \longrightarrow P_{\hat{\eta}}, \quad P_{p} \longrightarrow P_{\hat{p}}, \quad P_{q} \longrightarrow P_{\hat{q}},
$$

where $P_{q} \longrightarrow P_{\hat{q}}$ only exists for those nodes $q \in \underline{C}_{0}$ that are contained in the closure of a node of $\underline{C}_{K}$.

\footnotetext{
${ }^{7}$ In writing $\{\bar{w}\} \times_{W}(C / W, \mathbf{x}, f)$ we view $(C / W, \mathbf{x}, f)$ as an object over $\underline{W}$. In particular, this fibre product is a log curve over $\operatorname{Spec} \kappa(\bar{w})$ endowed with the log structure making the inclusion into $W$ strict.

${ }^{8}$ Alternatively, the following arguments indeed show that basicness holds on subsets of $\underline{W}$ which admit a chart $Q \rightarrow \mathcal{M}_{W}$ inducing an isomorphism $Q \simeq \overline{\mathcal{M}}_{W, \bar{w}}$ for some $\bar{w} \in|\Omega|$ (cf. [Ch] Proposition 3.5.2).
} 
For each generic point $\eta \in \underline{C}_{0}$ with generization $\hat{\eta} \in \underline{C}_{K}$ the homomorphism $\underline{f}^{*} \overline{\mathcal{M}}_{X} \rightarrow \overline{\mathcal{M}}_{C}$ defines a commutative square

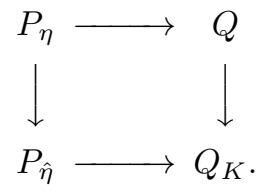

Moreover, if $q \in \operatorname{cl}(\hat{q})$, then $\rho_{q}$ maps to $\rho_{\hat{q}}$. We thus obtain a commutative diagram

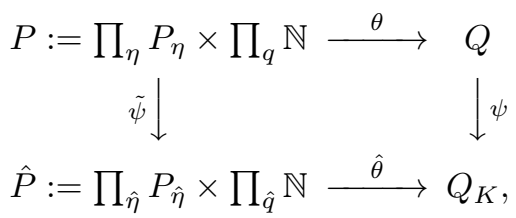

with $\theta$ the map defining $Q$ up to saturation and the vertical arrows the generization epimorphisms. If $q$ is a node not in the closure of some $\hat{q}$, then $\tilde{\psi}$ maps this copy of $\mathbb{N}$ to 0 .

As a generization homomorphism, $\psi$ induces an isomorphism $S^{-1} Q /\left(S^{-1} Q\right)^{\times} \rightarrow$ $Q_{K}$ with $S=\psi^{-1}(0)$ a face of $Q$. Similarly, $\tilde{\psi}$ induces an isomorphism

$$
\tilde{S}^{-1} P /\left(\tilde{S}^{-1} P\right)^{\times} \rightarrow \hat{P} \text {. }
$$

As the morphisms $\theta$ and $\hat{\theta}$ are local we have the relation

$$
\tilde{S}=\tilde{\psi}^{-1}(0)=\tilde{\psi}^{-1}\left(\hat{\theta}^{-1}(0)\right)=\theta^{-1}\left(\psi^{-1}(0)\right)=\theta^{-1}(S) .
$$

Recall from Construction 1.16 that $Q$ is the saturation of the image of $P$ in $P^{\mathrm{gp}} / R$, where $R$ is the saturation of the subgroup of $P^{\text {gp }}$ generated by certain elements $a_{q}(m)$, for all nodes $q$ and all $m \in P_{q}$. By the compatibility of types with generization (Lemma 1.11) $\tilde{\psi}^{\text {gp }}$ maps $R$ to the analogous subgroup $\hat{R} \subset \hat{P}$. Since by (1.16) $\hat{\theta}$ is surjective up to saturation, it remains to show that, up to saturation, $\hat{\theta}$ is the quotient of $\hat{P}$ by $\hat{R}$. Thus let $\hat{m}_{1}, \hat{m}_{2} \in \hat{P}$ with $\hat{\theta}\left(\hat{m}_{1}\right)=\hat{\theta}\left(\hat{m}_{2}\right)$. Let $m_{1}, m_{2}$ be lifts of $\hat{m}_{1}, \hat{m}_{2}$ to $P$. Then $\theta\left(m_{1}\right)-\theta\left(m_{2}\right) \in S^{\mathrm{gp}}$ (viewed in $Q^{\mathrm{gp}}$ ) for

$$
\psi\left(\theta\left(m_{1}\right)\right)=\hat{\theta}\left(\hat{m}_{1}\right)=\hat{\theta}\left(\hat{m}_{2}\right)=\psi\left(\theta\left(m_{2}\right)\right) .
$$

Thus there exist $h_{i} \in \theta^{-1}(S)$ such that $\theta\left(m_{1}+h_{1}\right)=\theta\left(m_{2}+h_{2}\right)$. Using $\theta^{-1}(S)=\tilde{S}$ from (1.17) we may replace $m_{i}$ by $m_{i}+h_{i}$ to achieve $\theta\left(m_{1}\right)=\theta\left(m_{2}\right)$. But then $m_{1}-m_{2} \in R$, and hence

$$
\tilde{\psi}\left(m_{1}\right)-\tilde{\psi}\left(m_{2}\right) \in \tilde{\psi}^{\mathrm{gp}}(R)=\hat{R},
$$

finishing the proof.

The next proposition establishes a universal property for basic stable log maps. This shows, in particular, that we do not lose any generality in imposing basicness. This result is not needed for the construction of log Gromov-Witten invariants, but is included for reassurance. It is also referred to in the comparison with Jun Li's moduli space in Corollary 6.2. As an auxiliary result we first treat the problem on the level of ghost sheaves.

Lemma 1.23. Let $(\underline{C} / \underline{W}, \mathbf{x})$ be a pre-stable curve and $\overline{\mathcal{M}}$ a fine saturated sheaf on $\underline{C}$. For each geometric point $\bar{w}: \operatorname{Spec} \kappa(\bar{w}) \rightarrow \underline{W}$ let a type $\mathbf{u}_{\bar{w}}$ of an object of $\underline{\operatorname{GS}}\left(\left.\overline{\mathcal{M}}\right|_{\underline{C}_{\bar{w}}}\right)$ be given such that the collection $\left(\mathbf{u}_{\bar{w}}\right)_{\bar{w}}$ of types is compatible with 
generization. Then the full subcategory $\underline{\operatorname{GS}}\left(\overline{\mathcal{M}},\left(\mathbf{u}_{\bar{w}}\right)\right)$ of objects of $\underline{\operatorname{GS}}(\overline{\mathcal{M}})$ that have type $\mathbf{u}_{\bar{w}}$ over the geometric point $\bar{w}$ has a universal object.

Proof. For geometric points this is the statement of Proposition 1.19, In the general case, diagram (1.16) in the proof of Proposition 1.22 shows that the fibrewise defined diagrams of ghost sheaves are compatible with generization. Hence they define the desired initial object in the category of diagrams of ghost sheaves.

Proposition 1.24. Any stable log map arises as the pull-back from a basic stable log map with the same underlying ordinary stable map. Both the basic stable log map and the morphism are unique up to unique isomorphism.

Proof. Let $(\pi: C \rightarrow W, \mathbf{x}, f)$ be the given stable log map, defining morphisms of log structures $\pi^{b}: \underline{\pi}^{*} \mathcal{M}_{W} \rightarrow \mathcal{M}_{C}$ and $f^{b}: f^{*} \mathcal{M}_{X} \rightarrow \mathcal{M}_{C}$. We consider the category of morphisms of $\log$ structures $\mathcal{M}_{W}^{\prime} \rightarrow \mathcal{M}_{W}$ on $\underline{W}$ and $\mathcal{M}_{C}^{\prime} \rightarrow \mathcal{M}_{C}, \underline{\pi}^{*} \mathcal{M}_{W}^{\prime} \rightarrow \mathcal{M}_{C}^{\prime}$, $f^{*} \mathcal{M}_{X} \rightarrow \mathcal{M}_{C}^{\prime}$ on $\underline{C}$, compatible with $\pi^{b}$ and $\overline{f^{b}}$ in the obvious way. The statement follows once we show that this category has an initial object, and that this object is a basic stable log map.

If $\mathcal{M}_{1} \rightarrow \mathcal{M}_{2}$ is a morphism of fine log structures on a scheme $Y$, then from the commutative diagram

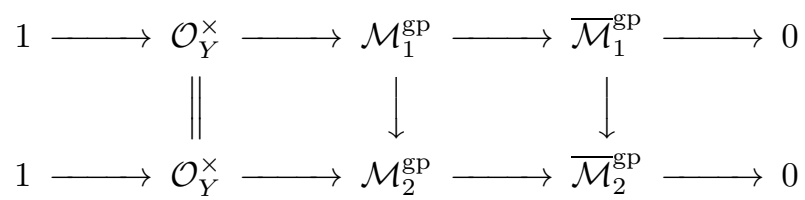

it follows that $\mathcal{M}_{1}=\mathcal{M}_{2} \times \overline{\mathcal{M}}_{2} \overline{\mathcal{M}}_{1}$. Moreover, a morphism of log structures with target $\mathcal{M}_{2}$ lifts to $\mathcal{M}_{1}$ if and only if this is true on the level of ghost sheaves. In particular, the functor defined by going over from a stable log map to the associated diagram of ghost sheaves is an equivalence from the present category to $\underline{\operatorname{GS}}\left(\overline{\mathcal{M}},\left(\mathbf{u}_{\bar{w}}\right)\right)$. Here $\mathbf{u}_{\bar{w}}$ is the type of the given stable log map $(C / W, \mathbf{x}, f)$ at the geometric point $\bar{w}$. The statement now follows from Lemma 1.23 .

Another remarkable property of basic stable log maps is that they do not admit non-trivial automorphisms that are the identity on the underlying ordinary stable maps. Stack-theoretically, this means that the forgetful map from the stack of basic stable log maps to the stack of ordinary stable maps is representable (Proposition 2.77). The statement is also useful for checking that the stack of stable log maps has a separated diagonal (Proposition 2.5).

Proposition 1.25. An automorphism $\varphi: C / W \rightarrow C / W$ of a basic stable log map $(\pi: C \rightarrow W, \mathbf{x}, f)$ with $\underline{\varphi}=\operatorname{id}_{\underline{C}}$ is trivial.

Proof. An automorphism of $C / W$ is an automorphism $\left(\underline{\varphi}, \varphi^{b}\right)$ of $C=\left(\underline{C}, \mathcal{M}_{C}\right)$ descending to an automorphism $\psi=\left(\mathrm{id}, \psi^{\mathrm{b}}\right)$ of $\left(\underline{W}, \mathcal{M}_{W}\right)$. It is an automorphism of $(C / W, \mathbf{x}, f)$ if $\underline{\varphi}(\mathbf{x})=\mathbf{x}$ and if it commutes with the morphism $f^{b}: \underline{f}^{*} \mathcal{M}_{X} \rightarrow \mathcal{M}_{C}$. Since $\underline{\varphi}=\operatorname{id}_{C}$ the latter condition means

$$
\varphi^{b} \circ f^{b}=f^{b} .
$$

We claim that it suffices to show $\psi^{b}=\operatorname{id}_{\mathcal{M}_{W}}$. In fact, let $U \subset \underline{C}$ be the complement of the set of special points (images of marked points or criticial points of $\underline{\pi}$ ). Because $\pi^{b}: \pi^{*} \mathcal{M}_{W} \rightarrow \mathcal{M}_{C}$ is an isomorphism on $U, \psi^{b}=\operatorname{id}_{\mathcal{M}_{W}}$ implies 
$\left.\varphi^{b}\right|_{U}=$ id. But by Theorem 1.1, $\mathcal{M}_{C}$ has no section with support on the set of special points. Hence $\varphi^{b}=\mathrm{id}_{\mathcal{M}_{C}}$.

Now on the level of ghost sheaves a basic stable log map is determined by the underlying ordinary stable map and the type. Moreover, there is no morphism between stable $\log$ maps of different types. This shows that $\underline{\varphi}=\mathrm{id}_{\underline{C}} \operatorname{implies}$ $\overline{\varphi^{b}}=\mathrm{id} \overline{\mathcal{M}}_{C}$. By strictness of $\pi: C \rightarrow W$ away from the special points this imples $\overline{\psi^{b}}=\mathrm{id}_{\overline{\mathcal{M}}_{W}}$. Thus $\psi^{b}$ has the form

$$
\psi^{b}(m)=h(\bar{m}) \cdot m
$$

for a homomorphism $h: \overline{\mathcal{M}}_{W} \rightarrow \mathcal{O}_{W}^{\times}$. Now if $\bar{w} \rightarrow \underline{W}$ is a geometric point, then by basicness, up to saturation, $\overline{\mathcal{M}}_{W, \bar{w}}$ is generated by the fibres $P_{\eta}$ of $f^{*} \overline{\mathcal{M}}_{X}$ at the generic points $\eta \in \underline{C}_{\bar{w}}$, and by one copy of $\mathbb{N}$ for each singular point of $\underline{C}_{\bar{w}}$. Strictness of $\left.\pi\right|_{U}$ together with (1.18) implies that $h$ is trivial on the part of $\overline{\mathcal{M}}_{W}$ generated by $P_{\eta}$. The factors of $\mathbb{N}$ are generated by the image of the basic log structure $\pi^{*} \mathcal{M}_{W}^{o} \rightarrow \mathcal{M}_{C}^{o}$ on the pre-stable curve $\underline{C} / \underline{W}$. But $\mathcal{M}_{C}^{o}$ has no non-trivial automorphism inducing the identity on $\underline{C}$ and on $\overline{\mathcal{M}_{C}^{o}}$. This is a direct consequence of the existence of basic log structures for pre-stable curves; see Appendix A. Hence $h=1$ also on this part. Finally note that a homomorphism from a fine monoid to a group is trivial if and only if it is trivial on its saturation. Hence $h$ is trivial and thus $\psi^{b}=\mathrm{id}_{\mathcal{M}_{W}}$ as remained to be shown.

\section{Algebraicity}

2.1. The stack of stable log maps. We are now ready to define the stack of stable log maps. We continue with the convention that $X$ is a log scheme over $S$, with the $\log$ structures defined on the Zariski sites. Remember also that all our $\log$ structures are defined over $S$, that is, they come with a morphism from the pull-back of $\mathcal{M}_{S}$ that is compatible with $\mathcal{M}_{S} \rightarrow \mathcal{O}_{\underline{S}}$. We endow $(\operatorname{Sch} / \underline{S})$ with the étale Grothendieck topology.

Definition 2.1. The stack of stable log maps to $X$ (over $S$ ) is the category $\tilde{\mathscr{M}}(X)=\tilde{\mathscr{M}}(X / S)($ Definition 1.6) ) together with the forgetful morphism $\tilde{\mathscr{M}}(X) \rightarrow$ $(\mathrm{Sch} / \underline{S})$ mapping $(C / W, \mathbf{x}, f)$ to $\underline{W}$. The full subcategory of basic stable log maps is denoted $\mathscr{M}(X)$.

Since the morphisms in $\tilde{\mathscr{M}}(X)$ and $\mathscr{M}(X)$ are given by cartesian diagrams of log smooth curves over the underlying base schemes, $\tilde{\mathscr{M}}(X) \rightarrow(\mathrm{Sch} / \underline{S})$ and $\mathscr{M}(X) \rightarrow$ $(\mathrm{Sch} / \underline{S})$ are fibred groupoids. As is customary, for any $a \in \tilde{\mathscr{M}}(X)$ over $\underline{W} \in$ $(\mathrm{Sch} / \underline{S})$ and $\varphi: \underline{V} \rightarrow \underline{W}$ we choose one morphism in $\tilde{\mathscr{M}}(X)$ covering $\varphi$ and denote it by $\varphi^{*} a \rightarrow a$.

Once we prove that $\tilde{\mathscr{M}}(X)$ is an algebraic stack, Proposition 1.22 shows that $\mathscr{M}(X)$ is also algebraic, for it is an an open substack of $\tilde{\mathscr{M}}(X)$. We therefore restrict attention to $\tilde{\mathscr{M}}(X)$ for most of this section.

Lemma 2.2. $\tilde{\mathscr{M}}(X)$ is a stack.

Proof. We verify Axioms (i) and (ii) in LaMB], Definition 3.1.

(i) We have to check the sheaf axioms for morphisms between two objects in $\tilde{\mathscr{M}}(X)$ over the same base scheme. This amounts to the following. Let $a_{i} \in$ $\tilde{\mathscr{M}}(X)_{\underline{W}}, i=1,2$, be two stable log maps with the same base scheme $\underline{W}$. Let 
$h: \underline{\tilde{W}} \rightarrow \underline{W}$ be an étale cover, $\tilde{\psi}: h^{*} a_{1} \rightarrow h^{*} a_{2}$ a morphism over $\underline{\tilde{W}}$ and $\operatorname{pr}_{i}:$ $\underline{\tilde{W}} \times \underline{W} \underline{\tilde{W}} \rightarrow \underline{W}$ for $i=1,2$ the projections. With $\pi:=h \circ \mathrm{pr}_{1}=h \circ \overline{p r}_{2}$ there are two morphisms

$$
\operatorname{pr}_{i}^{*} \tilde{\psi}: \pi^{*} a_{1} \longrightarrow \pi^{*} a_{2}, \quad i=1,2 .
$$

The sheaf axiom says that if these two morphisms agree, then there exists a unique morphism $\psi: a_{1} \rightarrow a_{2}$ with $\tilde{\psi}=h^{*} \psi$. For the underlying morphisms of schemes this follows from faithfully flat descent (SGA1, VIII, Theorem 5.2). Since on the domains we work with log structures in the étale topology the refinement to morphisms of log schemes is a tautology.

(ii) This axiom deals with descent for objects in $\tilde{\mathscr{M}}(X)$. On the underlying domain of the stable log map this follows by the sheaf property of the stack of prestable curves $\mathbf{M}$ (see Appendix $\underline{A}$ ). Then the underlying morphisms to $\underline{X}$ descend as in (i). Again the refinement to morphisms of log spaces is a tautology.

The rest of this section is devoted to proving algebraicity of $\tilde{\mathscr{M}}(X)$. Denote by $\mathscr{M}=\mathscr{M}_{S}$ the log stack over $S$ of (not necessarily basic) pre-stable marked log curves. In Appendix $\mathrm{A}$ we recall the folklore result that $\mathscr{M}$ is an algebraic stack locally of finite type over $\underline{S}$ (Proposition A.3). There is a forgetful morphism of stacks

$$
\tilde{\mathscr{M}}(X) \longrightarrow \mathscr{M}
$$

mapping a stable log map $(C / W, \mathbf{x}, f)$ to the pre-stable marked log curve $(C / W, \mathbf{x})$. Note that this functor is faithful, since a morphism of stable log maps is given by a morphism on the domains. Another forgetful morphism is to the algebraic stack of ordinary stable maps $\mathbf{M}(\underline{X})[\mathrm{BeMa}$ :

$$
\tilde{\mathscr{M}}(X) \longrightarrow \mathbf{M}(\underline{X}) .
$$

In the next subsection we will prove the following.

Proposition 2.3. $\tilde{\mathscr{M}}(X) \rightarrow \mathscr{M} \times \mathbf{M}(\underline{X})$ is representable and locally of finite presentation.

A direct consequence is the main result of this section.

Theorem 2.4. $\tilde{\mathscr{M}}(X)$ is an algebraic stack locally of finite type over $\underline{S}$.

Proof. By LaMB, Proposition 4.5(ii) algebraicity of $\tilde{\mathscr{M}}(X)$ follows from Proposition 2.3 . It is locally of finite type over $\underline{S}$ since $\mathscr{M}$ and $\mathbf{M}(\underline{X})$ are as well.

As is the case with $\mathscr{M}$, the stack $\tilde{\mathscr{M}}(X)$ is only an algebraic stack in the sense of $\mathrm{Ol2}$, p. 750. This definition drops the separatedness of the diagonal morphism (quasi-separatedness) from the definition in [LaMB]. In contrast, the open substack $\mathscr{M}(X)$ of $\tilde{\mathscr{M}}(X)$ does have a separated diagonal, so is an algebraic stack in the sense of $[\mathrm{LaMB}$ :

Proposition 2.5. The diagonal morphism $\Delta_{\mathscr{M}(X) / S}: \mathscr{M}(X) \longrightarrow \mathscr{M}(X) \times \mathscr{M}(X)$ is separated.

Proof. The statement amounts to the following: An automorphism of a stable log map $(C / W, \mathbf{x}, f)$ over an integral scheme $\underline{W}$ that is generically the identity is trivial. This is clearly true for the underlying ordinary stable map, and lifts to basic stable $\log$ maps by virtue of Proposition 1.25. 
As a corollary of Theorem 2.4 we obtain algebraicity of the open substack $\mathscr{M}(X)$ of $\tilde{\mathscr{M}}(X)$ of basic stable log maps. Moreover, $\mathscr{M}(X)$ carries a canonical log structure, that is, a factorization

$$
\mathscr{M}(X) \longrightarrow(\log / S) \longrightarrow(\mathrm{Sch} / \underline{S}),
$$

where $(\log / S)$ is the category of fine saturated $\log$ schemes over $S$ with strict morphisms.

Corollary 2.6. $\mathscr{M}(X)$ is an algebraic log stack with separated diagonal and locally of finite type over $\underline{S}$.

Proof. For the $\log$ structure, define the functor $\mathscr{M}(X) \longrightarrow(\log / S)$ by sending a stable log map $(C / W, \mathbf{x}, f)$ to the logarithmic base $W$.

Assuming Proposition 2.3 we can establish at this point some further properties of $\mathscr{M}(X)$.

Proposition 2.7. The forgetful morphism $\mathscr{M}(X) \rightarrow \mathbf{M}(\underline{X})$ of algebraic stacks is representable.

Proof. By Corollaire 8.1.2 in LaMB] we have to show that the diagonal morphism

$$
\mathscr{M}(X) \longrightarrow \mathscr{M}(X) \times_{\mathbf{M}(\underline{X})} \mathscr{M}(X)
$$

is a monomorphism. This amounts to the statement about automorphisms of basic stable log maps verified in Proposition 1.25.

Corollary 2.8. The algebraic stack $\mathscr{M}(X)$ is a Deligne-Mumford stack.

Proof. An algebraic stack representable over a Deligne-Mumford stack is itself a Deligne-Mumford stack. In fact, if $X \rightarrow \mathfrak{X}$ is an étale presentation of an algebraic stack and $\mathfrak{Y} \rightarrow \mathfrak{X}$ is representable, then $Y:=X \times \mathfrak{X} \mathfrak{Y} \rightarrow \mathfrak{Y}$ is also an étale surjection, and $Y$ is an algebraic space by representability.

2.2. Representability of spaces of log morphisms. We now prove Proposition 2.3. To avoid excessive underlining, in this subsection we change our convention and denote schemes or algebraic spaces by unadorned letters and use a dagger for log schemes, as in $X^{\dagger}=\left(X, \mathcal{M}_{X}\right)$. We have to show that if $W$ is a scheme 9 and $W \rightarrow \mathscr{M} \times \mathbf{M}(X)$ is a morphism, then the fibre product $W \times \mathscr{M} \times \mathbf{M}(X) \tilde{\mathscr{M}}(X)$ is an algebraic space locally of finite type over $W$. Explicitly, this means the following. The morphism $W \rightarrow \mathscr{M} \times \mathbf{M}(X)$ amounts to giving a pair $\left(\mathcal{W}^{\dagger}, f\right)$ with $\mathcal{W}^{\dagger}=\left(C^{\dagger} / W^{\dagger}, \mathbf{x}\right)$ a pre-stable marked log curve and $f: C \rightarrow X$ a morphism of schemes making $(C / W, \mathbf{x}, f)$ an ordinary stable map. For $V \rightarrow W$, the fibre category of $W \times \mathscr{M} \times \mathbf{M}(X) \tilde{\mathscr{M}}(X)$ over $V$ can be taken as the category with objects morphisms of $\log$ structures

$$
\left(f^{*} \mathcal{M}_{X}\right)_{V} \longrightarrow\left(\mathcal{M}_{C}\right)_{V}
$$

over $S^{\dagger}$ and with only the identity as morphisms. Here the index $V$ means pull-back via the base change morphism $C_{V}:=V \times_{W} C \rightarrow C$. Triviality of the automorphisms in this fibre category is due to the fact that $\tilde{\mathscr{M}}(X) \rightarrow \mathscr{M} \times \mathbf{M}(X)$ is faithful. Thus the question is about the representability of the functor of morphisms between two given $\log$ structures over $S^{\dagger}$ along the fibres of the proper morphism $C \rightarrow W$.

\footnotetext{
${ }^{9}$ We follow the usual convention to confuse a scheme and its associated stack
} 
Abstracting, now let $\pi: Y \rightarrow W$ be a proper morphism of schemes and let $\alpha_{i}: \mathcal{M}_{i} \rightarrow \mathcal{O}_{Y}, i=1,2$, be two fine saturated log structures on $Y$. Consider the functor

$$
\operatorname{LMor}_{Y / W}\left(\mathcal{M}_{1}, \mathcal{M}_{2}\right):(\mathrm{Sch} / W) \longrightarrow(\text { Sets })
$$

that on objects is defined by

$$
V \longmapsto\left\{\varphi:\left(\mathcal{M}_{1}\right)_{V} \rightarrow\left(\mathcal{M}_{2}\right)_{V} \text { morphism of log structures }\right\} .
$$

Then the statement that $W \times \mathscr{M} \times \mathbf{M}(X) \tilde{\mathscr{M}}(X)$ is an algebraic space 10 essentially is a special case of the following proposition.

Proposition 2.9. $\operatorname{LMor}_{Y / W}\left(\mathcal{M}_{1}, \mathcal{M}_{2}\right)$ is represented by an algebraic space locally of finite type over $W$.

The proof is provided, after some preparations, at the end of this subsection. This then finishes the proof of Proposition 2.3 in the case $S=$ Spec $\mathbb{k}$ with the trivial $\log$ structure. In the general case we have in addition two morphisms of $\log$ structures $\psi_{i}: \pi^{*} \mathcal{M}_{S} \rightarrow \mathcal{M}_{i}, \pi: C \rightarrow W$, the projection, and we need to restrict to those $\varphi:\left(\mathcal{M}_{1}\right)_{V} \rightarrow\left(\mathcal{M}_{2}\right)_{V}$ compatible with $\psi_{i}$. But by Proposition 2.9 composition with $\psi_{1}$ defines a morphism of algebraic spaces

$$
\operatorname{LMor}_{Y / W}\left(\mathcal{M}_{1}, \mathcal{M}_{2}\right) \longrightarrow \operatorname{LMor}_{Y / W}\left(\pi^{*} \mathcal{M}_{S}, \mathcal{M}_{2}\right) \text {. }
$$

Now $W \times \mathscr{M} \times \mathbf{M}(X) \tilde{\mathscr{M}}(X)$ arises as the fibre product with the morphism

$$
W \longrightarrow \operatorname{LMor}_{Y / W}\left(\pi^{*} \mathcal{M}_{S}, \mathcal{M}_{2}\right)
$$

defined by $\psi_{2}$, and is hence represented by an algebraic space. This finishes the proof of Proposition 2.3 also in the general case.

Remark 2.10. One problem in showing representability of $\operatorname{LMor}_{Y / W}\left(\mathcal{M}_{1}, \mathcal{M}_{2}\right)$ is that it is non-separated, essentially because the induced map $\overline{\mathcal{M}}_{1} \rightarrow \overline{\mathcal{M}}_{2}$ cannot be determined by its restriction to an open dense subset. As an example (cf. Ol2, Remark 3.12) consider the $\log$ structure $\mathcal{M}$ on $\mathbb{A}^{1}=\operatorname{Spec} \mathbb{k}[x]$ with chart

$$
\mathbb{N}^{2} \longrightarrow \mathbb{k}[x], \quad(a, b) \longmapsto x^{a+b} .
$$

The map $\mathbb{N}^{2} \rightarrow \mathbb{N}^{2},(a, b) \mapsto(b, a)$ induces a non-trivial automorphism of $\mathcal{M}$ that restricts to the identity on $\mathbb{A}^{1} \backslash\{0\}$.

To find an étale cover of the algebraic space representing $\operatorname{LMor}_{Y / W}\left(\mathcal{M}_{1}, \mathcal{M}_{2}\right)$ in Proposition 2.9 we thus first restrict the map $\bar{\varphi}$. To this end let $\bar{w} \rightarrow W$ be a geometric point and let

$$
\bar{\varphi}_{\bar{w}}:\left(\overline{\mathcal{M}}_{1}\right)_{\bar{w}} \rightarrow\left(\overline{\mathcal{M}}_{2}\right) \bar{w}
$$

be a choice of $\bar{\varphi}$ over one geometric fibre $Y_{\bar{w}}$ of $Y \rightarrow W$. Now since $\overline{\mathcal{M}}_{i}$ are fine sheaves the choice at a geometric point $\bar{x} \rightarrow Y$ determines $\bar{\varphi}$ at any generization $\bar{y}$ of $\bar{x}$. Moreover, if $\bar{y}$ specializes to some other point $\bar{z}$ such that the generization map $\overline{\mathcal{M}}_{i, \bar{z}} \rightarrow \overline{\mathcal{M}}_{i, \bar{y}}$ is an isomorphism, then $\bar{\varphi}$ is also determined at $\bar{z}$. Iterating the generization-specialization process we are lead to the following definition.

\footnotetext{
${ }^{10}$ As for algebraic stacks we have to drop the condition of quasi-separatedness from the definition of algebraic spaces ([Kt], Ch.II, Definition 1.1).
} 
Definition 2.11. Let $\overline{\mathcal{M}}$ be a fine sheaf on a scheme $Y$ and let $A \subset|Y|$ be a set of geometric points. We say $\bar{x} \in|Y|$ has property $\left(A_{\text {gen }}\right)$ with respect to $\overline{\mathcal{M}}$ if there exists a sequence $\bar{y}_{1}, \bar{z}_{1}, \ldots, \bar{y}_{r}, \bar{z}_{r} \in|Y|$ for some $r$ with the following properties:

$$
\begin{array}{ll}
\bar{y}_{1} \in A, \quad \bar{z}_{r}=\bar{x}, & \\
\bar{y}_{i} \in \operatorname{cl}\left(\bar{z}_{i}\right), & i=1, \ldots, r, \\
\bar{y}_{i} \in \operatorname{cl}\left(\bar{z}_{i-1}\right) & \text { and } \overline{\mathcal{M}}_{\bar{y}_{i}} \rightarrow \overline{\mathcal{M}}_{\bar{z}_{i-1}} \text { is an isomorphism, } i=2, \ldots, r .
\end{array}
$$

Thus the giving of $\bar{\varphi}$ on a closed subset $A \subset|Y|$ then determines $\bar{\varphi}$ also on the subset

$$
U_{A}:=\left\{\bar{x} \in|Y| \mid \bar{x} \text { fulfills }\left(A_{\text {gen }}\right)\right\}
$$

of $|Y|$. Note that by definition $U_{A}$ is closed under generization. Since $\overline{\mathcal{M}}$ is a fine sheaf it is also immediate that $U_{A}$ is a constructible subset of $|Y|$, and hence $U_{A} \subset|Y|$ is open.

Since the statement of Proposition 2.9 is local in $W$ and by properness of $Y \rightarrow W$ we may assume any point of $|Y|$ fulfills $\left(A_{\text {gen }}\right)$ for $A=Y_{\bar{w}}$ with respect both to $\overline{\mathcal{M}}_{1}$ and to $\overline{\mathcal{M}}_{2}$, that is, $U_{Y_{\bar{w}}}=Y$. Then for any $V \rightarrow W$ there is at most one $\bar{\varphi}:\left(\overline{\mathcal{M}}_{1}\right)_{V} \rightarrow\left(\overline{\mathcal{M}}_{2}\right)_{V}$ compatible with $\bar{\varphi}_{\bar{w}}$ under sequences of generization maps. Let us call such $\bar{\varphi}$ (or a lift $\varphi$ to a morphism of $\log$ structures) compatible with $\bar{\varphi}_{\bar{w}}$, and similarly for any $A \subset|Y|$. Note that $\bar{\varphi}_{\bar{w}}$ may not extend to $Y$, but it may do so after certain base changes.

We first treat the representability problem locally on $Y$, that is, for $Y=W$.

Lemma 2.12. Let $Y=W$ and suppose that there exists a closed subset $A \subset Y$ such that any $\bar{x} \in|Y|$ fulfills $\left(A_{\text {gen }}\right)$ with respect to both $\overline{\mathcal{M}}_{i}$ (Definition 2.11 ). Let

$$
\bar{\varphi}_{A}:\left(\overline{\mathcal{M}}_{1}\right)_{A} \longrightarrow\left(\overline{\mathcal{M}}_{2}\right)_{A}
$$

be a homomorphism of sheaves of monoids. Then the functor

$$
\operatorname{LMor}_{Y}^{\bar{\varphi}_{A}}:\left(Y^{\prime} \stackrel{f}{\longrightarrow} Y\right) \longmapsto\left\{\varphi:\left(Y^{\prime}, f^{*} \mathcal{M}_{2}\right) \rightarrow\left(Y^{\prime}, f^{*} \mathcal{M}_{1}\right) \mid \varphi \text { is compatible with } \bar{\varphi}_{A}\right\}
$$

is represented by a scheme $\operatorname{LMor}_{Y}^{\bar{\varphi}_{A}}$ of finite type and affine over $Y$.

Proof. It is sufficient to prove the statement on an étale open cover of $Y$, since we can then use descent for affine morphisms ([SGA1], VIII, Theorem 2.1) to obtain a scheme over $Y$. Thus we can assume that we in fact have charts $\psi_{i}: P_{i} \rightarrow$ $\Gamma\left(Y, \mathcal{M}_{i}\right)$ for the two $\log$ structures. We can also assume that $\bar{\varphi}_{A}$ is induced by a homomorphism of monoids $\bar{\varphi}_{A}: P_{1} \rightarrow P_{2}$.

Let $p_{1}, \ldots, p_{n} \in P_{1}$ be a generating set for $P_{1}$ as a monoid. Consider the sheaf of finitely generated $\mathcal{O}_{Y}$-algebras

$$
\mathcal{F}_{Y}:=\mathcal{O}_{Y}\left[P_{1}^{\mathrm{gp}}\right] /\left\langle\alpha_{1}\left(\psi_{1}\left(p_{i}\right)\right)-z^{p_{i}} \alpha_{2}\left(\psi_{2}\left(\bar{\varphi}_{A}\left(p_{i}\right)\right)\right) \mid 1 \leq i \leq n\right\rangle .
$$

Then the desired scheme is $\operatorname{LMor}_{Y}^{\bar{\varphi}_{A}}:=\operatorname{Spec} \mathcal{F}_{Y}$.

To see that this is the correct scheme, suppose $f: Y^{\prime} \rightarrow Y$ is given. We wish to show that giving a commutative diagram of schemes

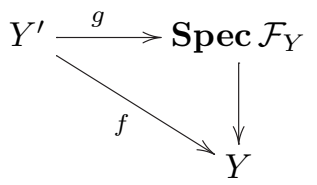


is equivalent to giving a $\log$ morphism $\varphi:\left(Y^{\prime}, f^{*} \mathcal{M}_{2}\right) \rightarrow\left(Y^{\prime}, f^{*} \mathcal{M}_{1}\right)$ which is the identity on $Y^{\prime}$ and lifts $f^{*}\left(\bar{\varphi}_{A}\right): f^{*} \overline{\mathcal{M}}_{1} \rightarrow f^{*} \overline{\mathcal{M}}_{2}$. Of course giving $g$ is equivalent to giving a section of $\left(\operatorname{Spec} \mathcal{F}_{Y}\right) \times_{Y} Y^{\prime}$ over $Y^{\prime}$. But

$$
\begin{aligned}
& \left(\operatorname{Spec} \mathcal{F}_{Y}\right) \times_{Y} Y^{\prime} \\
& \quad=\operatorname{Spec} \mathcal{O}_{Y^{\prime}}\left[P_{1}^{\mathrm{gp}}\right] /\left\langle f^{*}\left(\alpha_{1}\left(\psi_{1}\left(p_{i}\right)\right)\right)-z^{p_{i}} f^{*}\left(\alpha_{2}\left(\psi_{2}\left(\bar{\varphi}_{A}\left(p_{i}\right)\right)\right)\right) \mid 1 \leq i \leq n\right\rangle,
\end{aligned}
$$

and the latter scheme is $\mathbf{S p e c} \mathcal{F}_{Y^{\prime}}$ associated to the data $\left(Y^{\prime}, f^{*} \mathcal{M}_{1}\right),\left(Y^{\prime}, f^{*} \mathcal{M}_{2}\right)$ with charts $f^{*}\left(\psi_{i}\right)=f^{b} \circ \psi_{i}: P_{i} \rightarrow \Gamma\left(Y^{\prime}, f^{*} \mathcal{M}_{i}\right)$. Thus, without loss of generality, we can assume that $Y=Y^{\prime}$ and $f$ is the identity.

Now giving $\varphi:\left(Y, \mathcal{M}_{2}\right) \rightarrow\left(Y, \mathcal{M}_{1}\right)$ lifting $\bar{\varphi}_{A}$ is equivalent to specifying $\varphi^{b}$. In order for $\varphi^{b}$ to lift $\bar{\varphi}_{A}$, there must be a map $\eta: P_{1} \rightarrow \Gamma\left(Y, \mathcal{O}_{Y}^{\times}\right)$with the property that for all $p \in P_{1}$,

$$
\varphi^{b}\left(\psi_{1}(p)\right)=\eta(p) \cdot \psi_{2}\left(\bar{\varphi}_{A}(p)\right) .
$$

Giving $\eta$ completely determines $\varphi^{b}$. In addition, $\varphi^{b}$ is a homomorphism of monoids if and only if $\eta$ is a homomorphism, and since $\eta$ takes values in the group $\mathcal{O}_{Y}^{\times}$, specifying $\varphi^{b}$ is equivalent to specifying a section of $\mathbf{S p e c} \mathcal{O}_{Y}\left[P_{1}^{\mathrm{gp}}\right]$. Indeed, giving a section of $\operatorname{Spec} \mathcal{O}_{Y}\left[P_{1}^{\mathrm{gp}}\right]$ over $Y$ is the same as giving a morphism $Y \rightarrow \operatorname{Spec} \mathbb{k}\left[P_{1}^{\mathrm{gp}}\right]$, which in turn is the same as giving an element of $\operatorname{Hom}\left(P_{1}, \Gamma\left(Y, \mathcal{O}_{Y}^{\times}\right)\right)$.

Second, since $\varphi^{*}=\mathrm{id}$, we must have $\alpha_{1}=\alpha_{2} \circ \varphi^{b}$, so for each $p \in P_{1}$, we must have

$$
\alpha_{1}\left(\psi_{1}(p)\right)=\alpha_{2}\left(\varphi^{b}\left(\psi_{1}(p)\right)\right)=\eta(p) \cdot \alpha_{2}\left(\psi_{2}\left(\bar{\varphi}_{A}(p)\right)\right)
$$

If this holds for each $p_{i}$, it holds for all $p$. Thus a section of $\operatorname{Spec} \mathcal{O}_{Y}\left[P_{1}^{\text {gp }}\right]$ over $Y$ determines a morphism of log structures if and only if it lies in the subscheme determined by the equations

$$
\alpha_{1}\left(\psi_{1}\left(p_{i}\right)\right)-z^{p_{i}} \alpha_{2}\left(\psi_{2}\left(\bar{\varphi}_{A}\left(p_{i}\right)\right)\right),
$$

demonstrating the result.

Lemma 2.13. Let $Y \rightarrow W$ be a projective, separated morphism of schemes. Let $\bar{w} \rightarrow W$ be a geometric point, and assume any $\bar{x} \in|Y|$ fulfills $\left(A_{\text {gen }}\right)$ with respect to both $\overline{\mathcal{M}}_{i}$ for $A=Y_{\bar{w}}$ (Definition 2.11$)$. Then for a homomorphism $\bar{\varphi}_{A}:\left(\overline{\mathcal{M}}_{1}\right)_{\bar{w}} \rightarrow$ $\left(\overline{\mathcal{M}}_{2}\right)_{\bar{w}}$ of sheaves of monoids the functor

$$
\operatorname{LMor}_{Y / W}^{\bar{\varphi}_{A}}:(V \rightarrow W) \longmapsto\left\{\varphi:\left(\mathcal{M}_{1}\right)_{V} \rightarrow\left(\mathcal{M}_{2}\right)_{V} \mid \varphi \text { is compatible with } \bar{\varphi}_{A}\right\}
$$

is represented by a scheme $\operatorname{LMor}_{Y / W}^{\bar{\varphi}_{A}}$ of finite type over $W$.

Proof. Let $Z=\operatorname{LMor}_{Y}^{\bar{\varphi}_{A}}$. By Lemma 2.12, $\operatorname{LMor}_{Y / W}^{\bar{\varphi}_{A}}$ is isomorphic to the functor

$$
(V \rightarrow W) \longmapsto\left\{\text { sections of } Z_{V} \rightarrow Y_{V}\right\} \text {. }
$$

This is precisely the functor of sections $\prod_{Y / W}(Z / Y)$ discussed in Gt, p.267 (221-19) (see also [Nt), and it is represented by an open subscheme of $\mathrm{Hilb}_{Z / W}$. Furthermore, if $\mathcal{L}$ is a relatively ample line bundle on $Y$, then as $Z \rightarrow Y$ is affine, the pull-back of $\mathcal{L}$ to $Z$ is also (trivially) relatively ample over $W$. We use this ample line bundle to define Hilbert polynomials. Any section of $Z_{V} \rightarrow Y_{V}$ then must have the same Hilbert polynomial with respect to $\mathcal{L}$ as the Hilbert polynomial of $\mathcal{L}$ on $Y_{V}$, so in fact $\Pi_{Y / W}(Z / Y)$ defines an open subscheme of $\operatorname{Hilb}_{Z / W}^{P}$, for $P$ this fixed Hilbert polynomial. Thus $\Pi_{Y / W}(Z / Y)$ is represented by a scheme of finite type over $W$. 
We are now in position to prove Proposition 2.9.

Proof of Proposition 2.9. Since $\mathcal{M}_{1}$ and $\mathcal{M}_{2}$ are sheaves in the étale topology, LMor $_{Y / W}\left(\mathcal{M}_{1}, \mathcal{M}_{2}\right)$ is clearly a sheaf in the étale topology. It remains to prove the local representability statement of $\mathrm{Kt}$, Ch.II, Definition 1.1,b, for LMor $_{Y / W}\left(\mathcal{M}_{1}, \mathcal{M}_{2}\right)$. For any geometric fibre $A=Y_{\bar{w}}$ take an étale neighbourhood $U=U(\bar{w}) \rightarrow W$ of $\bar{w}$ such that $Y_{U}$ fulfills the assumptions of Lemma 2.13. Then for any $\bar{\varphi}_{\bar{w}}$ we have a scheme $\operatorname{LMor}_{Y_{U} / U}^{\bar{\varphi}_{\bar{w}}}$ of finite type over $W$. We claim that the natural functor

$$
Z:=\coprod_{\bar{\varphi}_{\bar{w}}} \operatorname{LMor}_{Y_{U} / U}^{\bar{\varphi}_{\bar{w}}} \longrightarrow \operatorname{LMor}_{Y / W}\left(\mathcal{M}_{1}, \mathcal{M}_{2}\right)
$$

is schematic and an étale surjection. This means explicitly that if $V$ is a scheme and $V \rightarrow \operatorname{LMor}_{Y / W}\left(\mathcal{M}_{1}, \mathcal{M}_{2}\right)$ is a morphism of stacks, then the fibre product in the sense of 2-categories $V \times{ }_{\operatorname{LMor}_{Y / W}\left(\mathcal{M}_{1}, \mathcal{M}_{2}\right)} Z$ is represented by a scheme, and the projection to $V$ is an étale surjection. We claim that for one $\operatorname{LMor}_{Y_{U} / U}^{\bar{\varphi}_{\bar{w}}}$ the representing scheme is an open subset of $V \times_{W} U$. In fact, the functor $V \rightarrow$ $\operatorname{LMor}_{Y / W}\left(\mathcal{M}_{1}, \mathcal{M}_{2}\right)$ says that we fix a morphism of $\log$ structures $\varphi:\left(\mathcal{M}_{1}\right)_{V} \rightarrow$ $\left(\mathcal{M}_{2}\right)_{V}$. Now a functor

$$
\psi: T \longrightarrow V \times \operatorname{LMor}_{Y / W}\left(\mathcal{M}_{1}, \mathcal{M}_{2}\right) \operatorname{LMor}_{Y_{U} / U}^{\bar{\varphi}_{\bar{w}}}
$$

from a scheme $T$ is nothing but (i) a morphism $T \rightarrow V$ and (ii) a morphism $T \rightarrow \operatorname{LMor}_{Y_{U} / U}^{\bar{\varphi}_{\bar{w}}}$ such that (iii) the compositions with the morphisms to LMor $_{Y / W}\left(\mathcal{M}_{1}, \mathcal{M}_{2}\right)$ coincide. Note that (i) provides a pull-back $\varphi_{T}:\left(\mathcal{M}_{1}\right)_{T} \rightarrow$ $\left(\mathcal{M}_{2}\right)_{T}$ of $\varphi$, (ii) gives a morphism of schemes $T \rightarrow U$ and a morphism of log structures $\varphi^{\prime}:\left(\mathcal{M}_{1}\right)_{T} \rightarrow\left(\mathcal{M}_{2}\right)_{T}$ with $\overline{\varphi^{\prime}}$ induced by $\bar{\varphi}_{\bar{w}}$, and (iii) says $\varphi^{\prime}=\varphi_{T}$. Thus $\psi$ is nothing but a factorization of the composition $T \rightarrow V \rightarrow W$ through $U$ along with the information that $\bar{\varphi}_{T}$ is induced by $\bar{\varphi}_{\bar{w}}$. Given $\varphi$, the latter condition defines an open subset of $V \times_{W} U$. Thus $\psi$ is canonically identified with a homomorphism from $T$ to an open subset of $V \times_{W} U$. This proves the claim. Note that since $U \rightarrow W$ is étale, then so is the projection $V \times_{W} U \rightarrow V$. Finally, surjectivity of $V \times_{\text {LMor }_{Y / W}\left(\mathcal{M}_{1}, \mathcal{M}_{2}\right)} Z \rightarrow V$ follows from the fact that we took the union over all $\bar{\varphi}_{\bar{w}}$.

\section{BOUNDEDNESS}

The aim of this section is to identify parts of $\mathscr{M}(X)$ that are of finite type. The main results are Theorem 3.9 in $\$ 3.1$ and Theorem 3.12 in $\$ 3.2$.

3.1. Finiteness of combinatorial types. In Definition 1.10 (2) we defined the type of a stable log map over a geometric point. It is given by the dual intersection graph $\Gamma_{\underline{C}}$ of the domain and data $\mathbf{u}=\left\{\left(u_{p}\right)_{p},\left(u_{q}\right)_{q}\right\}$. Morally the $u_{p}: P_{p} \rightarrow \mathbb{N}$ tell the order of contact with the toric divisors in a local chart for the log structure on $X$. Since by log smoothness these orders stay locally constant in families of stable log maps, they are part of the data distinguishing connected components of $\mathscr{M}(X)$. 
Definition 3.1. A class $\beta$ of stable $\log$ maps to $X$ consists of the following.

(i) The data $\beta$ of an underlying ordinary stable map, that is, the genus $g$ of $\underline{C}$, the number $k$ of marked points, and data $A$ bounding the degree, e.g., as described in $\mathrm{BeMa}$, p. 1211

(ii) Strict closed embeddings $Z_{1}, \ldots, Z_{k} \subset X$, with $\underline{Z}_{i}$ carrying the reduced induced scheme structure, together with sections $s_{i} \in \Gamma\left(\underline{Z}_{i},\left(\overline{\mathcal{M}}_{Z_{i}}^{\mathrm{gp}}\right)^{*}\right)$. We call $\beta$ maximal if none of the $s_{i}$ extends to any strictly larger subset of $X$.

A stable $\log \operatorname{map}(C / W, \mathbf{x}, f)$ is of class $\beta$ if the underlying ordinary stable map is of type $(g, k, A)$, and if for any $i$ we have $\operatorname{im}\left(\underline{f} \circ x_{i}\right) \subset \underline{Z}_{i}$ and for any geometric marked point $\bar{w} \rightarrow \underline{W}$ the map

$$
\overline{\mathcal{M}}_{Z_{i}, \underline{f}\left(x_{i}(\bar{w})\right)}=\left(\underline{f}^{*} \overline{\mathcal{M}}_{X}\right)_{x_{i}(\bar{w})} \stackrel{\bar{f}^{b}}{\longrightarrow} \overline{\mathcal{M}}_{C, x_{i}(\bar{w})}=\overline{\mathcal{M}}_{W, \underline{w}} \oplus \mathbb{N} \stackrel{\mathrm{pr}_{2}}{\longrightarrow} \mathbb{N}
$$

equals the germ of $s_{i}$ at $f\left(x_{i}(\bar{w})\right)$.

The substack of $\mathscr{M}(\overline{X)}$ of stable log maps of class $\beta$ is denoted $\mathscr{M}(X, \beta)$.

Note that the composition (3.1) is denoted $u_{p}$ in other parts of the text.

Remark 3.2. The matching condition $\operatorname{im}\left(f \circ x_{i}\right) \subset \underline{Z}_{i}$ clearly defines a closed algebraic substack of $\mathscr{M}(X)$, while the remaining conditions are open. In particular, $\mathscr{M}(X, \beta)$ is also an algebraic stack locally of finite type over $\underline{S}$.

Moreover, if $\beta$ is maximal, then $\mathscr{M}(X, \beta)$ is an open substack of $\mathscr{M}(X)$. In fact, the maximality condition says that if $z \in \underline{Z}_{i}$ lies in the closure of $y \in \underline{X} \backslash \underline{Z}_{i}$, then $s_{i}$ does not factor over the generization map

$$
\overline{\mathcal{M}}_{Z_{i}, z}^{\mathrm{gp}}=\overline{\mathcal{M}}_{X, z}^{\mathrm{gp}} \longrightarrow \overline{\mathcal{M}}_{X, y}^{\mathrm{gp}}
$$

for otherwise $s_{i}$ extends to $\underline{Z}_{i} \cup \operatorname{cl}(y)$. Thus, if $(C / W, \mathbf{x}, f)$ is a stable log map and the composition (3.1) equals the germ of $s_{i}$ at a point $\bar{w} \rightarrow \underline{W}$, then the same is true in a whole neighbourhood.

A necessary condition for boundedness of $\mathscr{M}(X, \beta)$ is that only finitely many types of stable $\log$ maps to $X$ of class $\beta$ occur. Unfortunately, we have been unable to prove this in complete generality; so far we have only been able to prove finiteness given certain assumptions on $X$. On the other hand, we also could not find an $X$ for which this finiteness does not hold. Thus we believe that the following definition is in fact empty, at least locally over $\mathbf{M}(\underline{X})$.

Definition 3.3. A class $\beta$ of stable $\log$ maps is called combinatorially finite if the set of types of stable log maps of class $\beta$ is finite.

Thus, in general, if one wishes to deal with log Gromov-Witten invariants for an $X$ for which we do not prove finiteness below, one will have to check finiteness for that $X$. However, we think that the cases discussed below will cover most, if not all, applications of log Gromov-Witten theory.

We fix in this section an ordinary stable map $\underline{f}: \underline{C} \rightarrow \underline{X}$ over Spec $\kappa$, and we consider all possible types of liftings of such a map to $f: C \rightarrow X$ over the standard $\log$ point $(\operatorname{Spec} \kappa, \mathbb{N})$.

We introduce several conditions a log scheme can satisfy which will be useful for proving boundedness.

\footnotetext{
${ }^{11}$ In $\mathrm{BeMa} A$ is defined by the degree function on the cone of isomorphism classes of ample invertible sheaves; if $\mathbb{k} \subset \mathbb{C}$ one might prefer prescribing a class in the singular homology group $H_{2}\left(\underline{X}_{\mathbb{C}}, \mathbb{Z}\right)$ of the associated complex variety $\underline{X}_{\mathbb{C}}$.
} 
Definition 3.4. We say a sheaf of monoids $\overline{\mathcal{M}}$ on (the Zariski site of) a scheme $\underline{Y}$ is almost-generated if the maps

$$
\operatorname{Hom}\left(\overline{\mathcal{M}}_{y}, \mathbb{R}_{\geq 0}\right) \rightarrow \operatorname{Hom}\left(\Gamma(\underline{Y}, \overline{\mathcal{M}}), \mathbb{R}_{\geq 0}\right)
$$

are injective for all $y \in \underline{Y}$. We say a log scheme $Y$ is almost-generated if $\overline{\mathcal{M}}_{Y}$ is almost-generated.

We say a sheaf of monoids $\overline{\mathcal{M}}$ on a scheme $\underline{Y}$ is quasi-generated if

$$
\operatorname{Hom}\left(\overline{\mathcal{M}}_{y}, \mathbb{R}\right) \rightarrow \operatorname{Hom}\left(\Gamma\left(\underline{Y}, \overline{\mathcal{M}}^{\mathrm{gp}}\right), \mathbb{R}\right)
$$

are injective for all $y \in \underline{Y}$. We say a log scheme $Y$ (with a log structure on the Zariski site) is quasi-generated if $\overline{\mathcal{M}}_{y}$ is quasi-generated.

Remark 3.5. (1) If $\overline{\mathcal{M}}$ is a sheaf of fine monoids, then being almost-generated is equivalent to saying that for any $y \in \underline{Y}$ the image of the restriction map $\Gamma(\underline{Y}, \overline{\mathcal{M}}) \rightarrow$ $\overline{\mathcal{M}}_{y}$ spans $\overline{\mathcal{M}}_{y}^{\mathrm{gp}} \otimes_{\mathbb{Z}} \mathbb{R}$. In fact, write $P=\overline{\mathcal{M}}_{y}$ and $Q=\operatorname{im}\left(\Gamma(\underline{Y}, \overline{\mathcal{M}}) \rightarrow \overline{\mathcal{M}}_{y}\right) \subset P$. If $\overline{\mathcal{M}}$ is almost-generated, then $\operatorname{Hom}\left(P, \mathbb{R}_{\geq 0}\right) \rightarrow \operatorname{Hom}\left(Q, \mathbb{R}_{\geq 0}\right)$ is injective. But then, since $P$ and $Q$ are fine monoids, also the induced map of associated groups

$$
\operatorname{Hom}(P, \mathbb{R})=\operatorname{Hom}\left(P, \mathbb{R}_{\geq 0}\right)^{\mathrm{gp}} \longrightarrow \operatorname{Hom}\left(Q, \mathbb{R}_{\geq 0}\right)^{\mathrm{gp}}=\operatorname{Hom}(Q, \mathbb{R})
$$

is injective. Thus $Q$ spans $P^{\mathrm{gp}} \otimes_{\mathbb{Z}} \mathbb{R}$.

Conversely, if $\operatorname{Hom}(P, \mathbb{R}) \rightarrow \operatorname{Hom}(Q, \mathbb{R})$ is injective then so is the restriction to $\operatorname{Hom}\left(P, \mathbb{R}_{\geq 0}\right)$.

(2) Similarly, a sheaf of fine monoids $\overline{\mathcal{M}}$ is quasi-generated iff for any $y \in \underline{Y}$ the image of $\Gamma\left(\underline{Y}, \overline{\mathcal{M}}^{\mathrm{gp}}\right) \rightarrow \overline{\mathcal{M}}_{y}^{\mathrm{gp}}$ spans $\overline{\mathcal{M}}_{y}^{\mathrm{gp}} \otimes_{\mathbb{Z}} \mathbb{R}$.

Examples 3.6. (1) The condition that $X$ be Deligne-Faltings arises in the work of Abramovich and Chen ACGM], Ch. This means that there is a surjection $\underline{\mathbb{N}}^{r} \rightarrow \overline{\mathcal{M}}_{X}$ for some $r$. The condition of $X$ being almost-generated is strictly weaker. For example, suppose $\underline{X}$ is a surface which is singular only at a point $p \in \underline{X}$, where $\underline{X}$ has an $A_{e-1}$ singularity, $e \geq 2$. Let $D=D_{1} \cup D_{2}$ be a divisor in $\underline{X}$ with $D_{1}, D_{2}$ irreducible and $D_{1} \cap D_{2}=\{p, q\}$, where $q \in \underline{X}$ is a smooth point of $\underline{X}$. Assume that near $p$ we have $\underline{X}$ locally given by the equation $x y=t^{e}$, and $D_{1} \cup D_{2}$ is locally given by $t=0$. Let $\underline{X}$ be given the divisorial log structure induced by $D$. Then one checks easily that $\Gamma\left(\underline{X}, \overline{\mathcal{M}}_{X}\right) \cong \overline{\mathcal{M}}_{X, p}=S_{e}$, the submonoid of $\mathbb{N}^{2}$ generated by $(e, 0),(0, e)$, and $(1,1)$. In particular, $\Gamma\left(\underline{X}, \overline{\mathcal{M}}_{X}\right) \rightarrow \overline{\mathcal{M}}_{X, q}=\mathbb{N}^{2}$ is not surjective. Thus $X$ cannot be Deligne-Faltings. However, one checks easily that $X$ is almost-generated.

On the other hand, Deligne-Faltings log structures are always almost-generated, as a surjection $P=\mathbb{N}^{r} \rightarrow \overline{\mathcal{M}}_{x}$ yields an injection $\operatorname{Hom}\left(\overline{\mathcal{M}}_{x}, \mathbb{R}_{\geq 0}\right) \rightarrow \operatorname{Hom}\left(P, \mathbb{R}_{\geq 0}\right)$.

(2) It is not clear that being quasi-generated is weaker than being almostgenerated. However, in some common situations, it is easier to check. In fact, it suffices to find a group $M$ and a map $M \rightarrow \Gamma\left(\underline{Y}, \overline{\mathcal{M}}^{\mathrm{gp}}\right)$ such that for all $y \in \underline{Y}$ the induced map

$$
\operatorname{Hom}\left(\overline{\mathcal{M}}_{y}, \mathbb{R}\right) \rightarrow \operatorname{Hom}(M, \mathbb{R})
$$

is injective. For example, if $X$ is a toric variety with the divisorial log structure defined by the toric divisors and $M$ is the character lattice, there is a natural surjection $\underline{M} \rightarrow \overline{\mathcal{M}}_{X}^{\mathrm{gp}}$. 
(3) If $X$ is almost-generated (quasi-generated), and $\underline{f}: \underline{C} \rightarrow \underline{X}$ is an ordinary stable map of curves, then the pull-back $\log$ structure $\underline{f}^{*} \mathcal{M}_{X}$ on $\underline{C}$ is almostgenerated (quasi-generated).

(4) Suppose we are given an ordinary stable map $f: \underline{C} \rightarrow \underline{X}$ and the dual intersection graph of $\underline{C}$ is a tree, for example, if $g(C)=0$. Then the pull-back log structure $\underline{f}^{*} \mathcal{M}_{X}$ is almost-generated. In fact, even better, the map $\Gamma\left(\underline{C}, \underline{f^{*}} \overline{\mathcal{M}}_{X}\right) \rightarrow$ $\left(f^{*} \overline{\mathcal{M}}_{X}\right)_{x}$ is surjective for every $x \in \underline{C}$, so the pull-back log structure is DeligneFaltings.

Indeed, with these assumptions, the sheaf $f^{*} \overline{\mathcal{M}}_{X}$ is entirely determined by the stalks $P_{\eta}$ and $P_{x}$ for special points $x$, along with uniquely determined generization maps $\chi_{\eta, x}: P_{x} \rightarrow P_{\eta}$ for every distinguished point $x$. To specify a section of $\underline{f}^{*} \overline{\mathcal{M}}_{X}$, we just need to specify elements $s_{x} \in P_{x}$ for all $x \in \underline{C}$ such that $\chi_{\eta, x}\left(s_{x} \overline{)}=s_{\eta}\right.$ for $x \in D, D:=\operatorname{cl}(\eta) \subset \underline{C}$. Now picking a point $x \in D$, and $s_{x} \in P_{x}$, set $s_{\eta}=\chi_{\eta, x}\left(s_{x}\right)$. For every other point $x^{\prime} \in D, x^{\prime} \neq x$, the generization map $\chi_{\eta, x^{\prime}}$ is always surjective, so we can choose $s_{x^{\prime}} \in P_{x^{\prime}}$ with $\chi_{\eta, x^{\prime}}\left(s_{x^{\prime}}\right)=s_{\eta}$. Some of these points $x^{\prime}$ will be double points, hence allowing us to define $s_{\eta^{\prime}}$ for other generic points $\eta^{\prime}$. Continuing in this fashion, using the fact there are no cycles in the dual intersection graph of $\underline{C}$, gives us a section of $\underline{f}^{*} \overline{\mathcal{M}}_{X}$ whose germ at $x$ is the given $s_{x}$.

Our arguments hinge on the following finiteness result from tropical geometry:

Proposition 3.7. Fixing a graph $\Gamma$, a lattice $N$, and weight vectors $u_{(v, E)} \in N$ for every non-compact edge $E$ of $\Gamma$, there are only a finite number of types of tropical curves with target $N_{\mathbb{R}}$ (as in Definition 1.12 ) with this given $\Gamma$ and $u_{(v, E)}$.

Proof. This is a weaker result than [NiSi], Proposition 2.1.

Theorem 3.8. Let $(\underline{C} / \operatorname{Spec} \kappa, \mathbf{x}, \underline{f})$ be an ordinary stable map and suppose $\overline{\mathcal{M}}:=$ $f^{*} \overline{\mathcal{M}}_{X}$ is almost-generated. Then there is only a finite number of types of log curves with the given underlying ordinary stable map.

Proof. Let

$$
M=\Gamma(\underline{C}, \overline{\mathcal{M}})^{\mathrm{gp}} .
$$

Let $N=\operatorname{Hom}(M, \mathbb{Z}), N_{\mathbb{R}}=N \otimes_{\mathbb{Z}} \mathbb{R}$. Clearly $M$ is spanned by the submonoid $\Gamma(\underline{C}, \overline{\mathcal{M}}) \subset M$. Thus also the dual submonoid $\Gamma(\underline{C}, \overline{\mathcal{M}})^{\vee} \subset N$ spans the dual space $N$, and such dual submonids are also sharp. In particular, $\Gamma(\underline{C}, \overline{\mathcal{M}})^{\vee}$ coincides with $K \cap N$ for some strictly convex rational polyhedral cone $K$ in $N_{\mathbb{R}}$.

Observe that since we can pull-back any stable log map to a standard log point it is enough to bound the types of tropical curves over standard log points. We thus consider now a stable log map $(C /(\operatorname{Spec} \kappa, \mathbb{N}), \mathbf{x}, f)$ over the standard log point. As in 1.4 this determines data $V_{\eta}, e_{q}, u_{p}, u_{q}$. The intersection graph $\Gamma_{\underline{C}}$ of $\underline{C}$ along with the data $\left\{\left(u_{p}\right),\left(u_{q}\right)\right\}$ is the type of $(C /(\operatorname{Spec} \kappa, \mathbb{N}), \mathbf{x}, f)$ by pull-back. Similarly to Discussion 1.13 we now look at the associated tropical curve. By the definition of almost-generated, we obtain for all $x \in \underline{C}$ inclusions

$$
\operatorname{Hom}\left(P_{x}, \mathbb{R}_{\geq 0}\right) \hookrightarrow K .
$$

Thus $V_{\eta} \in P_{\eta}^{\vee}, u_{p} \in P_{p}^{\vee}$ live naturally in $K$ and in fact in the monoid $\Gamma(\underline{C}, \overline{\mathcal{M}})^{\vee} \subset$ $N$. We also have, for each irreducible component $D=\operatorname{cl}(\eta)$ of $\underline{C}$, a map $\tau_{\eta}^{X}$ : $\Gamma\left(\tilde{D}, g^{*} \overline{\mathcal{M}}\right) \rightarrow \mathbb{Z}$, where $g: \tilde{D} \rightarrow \underline{C}$ is the normalization of $D$ followed by inclusion 
into $\underline{C}$; see (1.10). We then have a composition of the pull-back map on sections with $\bar{\tau}_{\eta}^{X}$ :

$$
M \rightarrow M_{\eta}:=\Gamma\left(\tilde{D}, g^{*} \overline{\mathcal{M}}\right)^{\mathrm{gp}} \stackrel{\tau_{\eta}^{X}}{\longrightarrow} \mathbb{Z} .
$$

This composition determines an element of $N$ which we also denote by $\tau_{\eta}^{X}$.

To build a tropical curve from this data add to $\Gamma_{\underline{C}}$ a number of unbounded edges: for each vertex $v_{\eta}$ we attach an unbounded edge, $E_{\eta}$, to $v_{\eta}$. We then define $h: \Gamma_{\underline{C}} \rightarrow N_{\mathbb{R}}$ by

$$
h\left(v_{\eta}\right)=V_{\eta} ;
$$

each edge $E_{q}$ with endpoints $v_{\eta_{1}}$ and $v_{\eta_{2}}$ is mapped to the line segment joining $V_{\eta_{1}}$ and $V_{\eta_{2}}$; and each edge $E_{p}$ with endpoint $v_{\eta}$ is mapped to the ray with endpoint $V_{\eta}$ with direction $u_{p}$. Finally, we map the ray $E_{\eta}$ to the ray with endpoint $V_{\eta}$ in the direction defined by $\tau_{\eta}^{X}$. (If $\tau_{\eta}^{X}=0$, the edge is contracted).

To give this the structure of a tropical curve, one also needs to assign integral weight vectors to each flag $(v, E)$ of $\Gamma_{\underline{C}}$. If $E=E_{q}$, we assign the vector $\pm u_{q}$, with the sign chosen so that $\pm u_{q}$ points away from $h(v)$. If $E=E_{p}$, we associate the vector $u_{p}$, and if $E=E_{\eta}$, we associate the vector $\tau_{\eta}^{X}$. These associated vectors are integral tangent vectors to the image of the corresponding edge. Then the tropical curve balancing condition is just the statement that for a given $\eta$,

$$
\tau_{\eta}^{X}+\sum_{x} u_{x}=0
$$

where the sum is over all special points $x \in \operatorname{cl}(\eta)$. But this is precisely the image of the equation of Proposition 1.15] in $M_{\eta}^{\vee}$, under the map $M_{\eta}^{\vee} \rightarrow N$. Thus $h$ defines a balanced tropical curve in $N_{\mathbb{R}}$.

Summing the balancing condition over all vertices gives a global balancing condition involving all unbounded edges. Namely, in $N$, we have

$$
\sum_{\eta} \tau_{\eta}^{X}+\sum_{p} u_{p}=0
$$

where $\eta$ runs over all generic points of $\underline{C}$ and $p$ runs over all marked points. Now $\sum_{\eta} \tau_{\eta}^{X} \in N$ is given, completely specified by the original stable map $\underline{f}: \underline{C} \rightarrow \underline{X}$ and independent of $f^{b}$, and $u_{p} \in \Gamma(\underline{C}, \overline{\mathcal{M}})^{\vee}$, which as observed at the beginning of the proof, is the set of integral points of a strictly convex cone in $N_{\mathbb{R}}$. Thus there is only a finite number of possibilities for writing $-\sum_{\eta} \tau_{\eta}^{X}$ as a sum of such $u_{p}$ 's. This shows finiteness of the choices of the $u_{p}$ 's.

For given $\left(u_{p}\right)_{p}$ and $\tau_{\eta}^{X}$, Proposition 3.7 shows there is only a finite number of possible combinatorial types of this tropical curve. This means there is only a finite number of possibilities for the vectors $u_{q} \in N$. On the other hand, these vectors are images of $u_{q} \in\left(P_{q}^{\mathrm{gp}}\right)^{*}$. Since $\left(P_{q}^{\mathrm{gp}}\right)^{*}$ injects into $N$, we conclude we only have a finite number of allowable types.

We have a slightly weaker result in the quasi-generated case, where we need to fix the $u_{p}$ 's, that is, the class.

Theorem 3.9. In the situation of Theorem 3.8 suppose that $\overline{\mathcal{M}}=f^{*} \overline{\mathcal{M}}_{X}$ is only quasi-generated. Then if the $u_{p}$ 's are fixed, there is only a finite number of types of log curves with the given underlying ordinary stable map. 
Proof. The argument is essentially the same as the proof of the previous theorem, this time taking $M=\Gamma\left(\underline{C}, \overline{\mathcal{M}}^{\mathrm{gp}}\right)$. Then via the same construction, one obtains a tropical curve in $N_{\mathbb{R}}$. However, one no longer has all $u_{p}$ living in a strictly convex cone in $N_{\mathbb{R}}$, so we cannot use this to control the vectors $u_{p}$. However, if we assume the $u_{p}$ are given, then Proposition 3.7 still gives the needed finiteness.

We next address what can be accomplished in more general situations. In Appendix $\mathrm{B}$ we introduce the tropicalization $\operatorname{Trop}(X)$ of $X$ as a natural target space for tropical curves. While this quite generally provides a tropical curve, it can be difficult to use the balancing condition in the often strange space $\operatorname{Trop}(X)$ in a useful way to prove finiteness.

Here is a special case that we state without proof, where it is still possible to prove finiteness. This is likely to be a good model for the type of application we have in mind, in which one considers varieties degenerating to unions of reasonably simple varieties.

Theorem 3.10. Let $\underline{X}$ be a scheme with $D \subset \underline{X}$ a divisor inducing a divisorial log structure on $\underline{X}$. Suppose this makes $X$ a fine, saturated log scheme, log smooth over Spec $\kappa$, and suppose furthermore:

(1) For each irreducible component $\underline{Y}$ of $D$, the restriction of the log structure of $X$ to $\underline{Y}$ is almost-generated. Furthermore, for $y \in \underline{Y}$, the inclusion $\operatorname{Hom}\left(\overline{\mathcal{M}}_{X, y}, \mathbb{R}_{\geq 0}\right) \rightarrow \operatorname{Hom}\left(\Gamma\left(\underline{Y}, \overline{\mathcal{M}}_{X}\right), \mathbb{R}_{\geq 0}\right)$ is an inclusion of faces 12

(2) $X$ is monodromy free (Definition B.2).

Then given an ordinary stable map $\underline{f}: \underline{C} \rightarrow \underline{X}$ and a collection of $u_{p} \in P_{p}^{\vee}$ for $p \in \underline{C}$ marked points, there is only a finite number of possible types of log curves with the given underlying ordinary stable map.

We were also able to prove boundedness without any further hypotheses in the case of genus one, also stated here without proof.

Theorem 3.11. Let $(\underline{C} / \operatorname{Spec} \kappa, \underline{x}, \underline{f})$ be an ordinary stable map and suppose the dual intersection graph $\Gamma_{\underline{C}}$ has genus at most one. Then there is only a finite number of types of log curves with the given underlying ordinary stable map.

3.2. Stable log maps of constant type and boundedness. Here is the main result of this section.

Theorem 3.12. For any combinatorially finite class $\beta$ of stable log maps to $X$, the algebraic stack $\mathscr{M}(X, \beta)$ is of finite type over $\underline{S}$.

By Corollary 2.6 we already know that $\mathscr{M}(X) \rightarrow \underline{S}$ is locally of finite type. Moreover, the stack of ordinary stable maps of fixed class $\mathbf{M}(\underline{X}, \beta)$ is of finite type over $\underline{S}$. So to finish the proof of Theorem 3.12 it remains to show that for any morphism $\underline{W} \rightarrow \mathbf{M}(\underline{X})$ from a quasi-compact scheme $\underline{W}$ the fibre product $\underline{W} \times{ }_{\mathbf{M}(\underline{X})} \mathscr{M}(X, \beta)$ is quasi-compact. By LaMB], Corollaire 5.6.3 this is equivalent to showing that the topological space of geometric points $\left|\underline{W} \times_{\mathbf{M}(\underline{X})} \mathscr{M}(X, \beta)\right|$ is quasi-compact. We do this by a stratawise approach.

Definition 3.13. A weak covering of a topological space $Z$ is a collection of subsets $\left\{A_{i}\right\}_{i \in I}$ with the following property: For any $z \in Z$ there exists $i \in I$ with $\operatorname{cl}(z) \cap$ $A_{i} \neq \emptyset$.

\footnotetext{
${ }^{12}$ Note this holds, for example, if the $\log$ structure on $Y$ is Deligne-Faltings.
} 
Lemma 3.14. Let $Z$ be a topological space weakly covered by finitely many quasicompact subsets $A_{i} \subset Z, i \in I$. Then $Z$ is quasi-compact.

Proof. Let $U_{j} \subset Z, j \in J$, be open subsets covering $Z$. By quasi-compactness of the $A_{i}$, for any $i \in I$ there exist finitely many $U_{j}$ covering $A_{i}$. Hence, since $I$ is finite, there exists a finite subset $J^{\prime} \subset J$ with $A_{i} \subset \bigcup_{j \in J^{\prime}} U_{j}$, for any $i$. We claim $Z=\bigcup_{j \in J^{\prime}} U_{j}$. In fact, let $z \in Z$. Then by the weak covering assumption there exists $i \in I$ with $\operatorname{cl}(z) \cap A_{i} \neq \emptyset$. Thus by the choice of $J^{\prime} \subset J$ there exists $j \in J^{\prime}$ with $\operatorname{cl}(z) \cap U_{j} \neq \emptyset$, and then $z \in U_{j}$ for $U_{j} \subset Z$ is open. Thus already finitely many of the $U_{j}$ cover $Z$.

Our strata $A_{i}$ will be defined by taking locally trivial families of ordinary stable maps.

Definition 3.15. An ordinary stable map $(\underline{\pi}: \underline{C} \rightarrow \underline{W}, \mathbf{x}, f)$ over an integral scheme $\underline{W}$ is called combinatorially constant if the following conditions are satisfied, where we write $\mathcal{M}:=\underline{f}^{*} \mathcal{M}_{X}$ as usual.

(1) If $g: \underline{\tilde{C}} \rightarrow \underline{C}$ is the normalization, then the composition $\underline{\pi} \circ g$ is a smooth map, and there are pairwise different sections $y_{q}$ of $\underline{\pi}$ with $\bigcup_{q} \operatorname{im}\left(y_{q}\right)=$ $\operatorname{crit}(\underline{\pi})$.

(2) Each irreducible component $\underline{C}_{\eta} \subset \underline{C}$ is geometrically connected and there is a section $\sigma_{\eta}$ of $\underline{\pi} \underline{C}_{\eta}$ with $\operatorname{im}\left(\sigma_{\eta}\right)$ disjoint from $\operatorname{im}(\mathbf{x})$ and from $\operatorname{crit}(\underline{\pi})$.

(3) For any $i, q$ the sheaves $x_{i}^{*} \overline{\mathcal{M}}$ and $y_{q}^{*} \overline{\mathcal{M}}$ are constant, and there are charts of $\log$ structures on $\underline{W}$,

$$
\underline{P_{\eta}} \longrightarrow \sigma_{\eta}^{*} \mathcal{M}, \quad \prod_{q} \underline{\mathbb{N}} \longrightarrow \mathcal{M}_{W}^{0},
$$

inducing isomorphisms $P_{\eta} \simeq \sigma_{\eta}^{*} \overline{\mathcal{M}}$ and $\prod_{q} \underline{\mathbb{N}} \simeq \overline{\mathcal{M}}_{W}^{0}$. Here $\mathcal{M}_{W}^{0}$ is the basic log structure for the pre-stable curve $\underline{C} / \underline{W}$.

Note that (3) defines isomorphisms $\sigma_{\eta}^{*} \mathcal{M} \simeq \underline{P}_{\eta} \times \mathcal{O}_{W}^{\times}$and $\mathcal{M}_{W}^{0} \simeq \prod_{q} \underline{\mathbb{N}} \times \mathcal{O}_{W}^{\times}$. Note also that for a combinatorially constant stable map $(\underline{C} / \underline{W}, \mathbf{x}, f)$ the existence of the sections $\sigma_{\eta}$ and Stein factorization imply that the geometric fibres of $\left.\underline{\pi} \circ g\right|_{\underline{C}_{\eta}}$ are connected. Thus all geometric fibres of $\underline{\pi}$ have the same dual intersection graph $\Gamma$ as the generic fibre, and $\underline{C}$ is obtained by gluing together families of smooth, connected curves along pairs of sections. Moreover, a type $\mathbf{u}=\left\{\left(u_{p}\right),\left(u_{q}\right)\right\}$ at the generic fibre induces a type for the fibre over every geometric point $\bar{w} \rightarrow \underline{W}$. By abuse of notation we call $\mathbf{u}$ a type for $(\underline{C} / \underline{W}, \mathbf{x}, \underline{f})$.

Let $\underline{W} \rightarrow \mathbf{M}(\underline{X})$ be the morphism defined by a combinatorially constant stable map $\mathfrak{f}=(\underline{C} / \underline{W}, \mathbf{x}, \underline{f})$. Then $\underline{W} \times{ }_{\mathbf{M}(\underline{X})} \mathscr{M}(X)$ is isomorphic as a stack to the (nonfull) subcategory $\mathscr{\mathscr { M }}(X, \mathfrak{f}) \subset \mathscr{M}(X)$ with objects stable log maps with underlying ordinary stable map obtained by pull-back from $\mathfrak{f}$, and with morphisms on the underlying schemes induced by the identity on $\mathfrak{f}$. Now the type of a stable log map in $\mathcal{M}(X, \mathfrak{f})$ is locally constant. Hence we have a decomposition into disjoint open substacks

$$
\underline{W} \times{ }_{\mathbf{M}(\underline{X})} \mathscr{M}(X) \simeq \coprod_{\mathbf{u}} \mathscr{M}(X, \mathfrak{f}, \mathbf{u})
$$

according to the type $\mathbf{u}$ for $\mathfrak{f}$.

The following lemma shows that provided $\underline{W}$ is reduced, the log structure on the base of a stable log map in $\mathscr{M}(X, \mathfrak{f}, \mathbf{u})$ is locally constant. 
Lemma 3.16. Let $W$ be a reduced scheme with a fine log structure $\mathcal{M}$ such that $\overline{\mathcal{M}}$ is a constant sheaf. Then étale locally, $\mathcal{M} \simeq O_{\underline{W}}^{\times} \times \overline{\mathcal{M}}$.

Proof. Let $Q$ be the stalk of $\overline{\mathcal{M}}$. Then a chart for $\mathcal{M}$ takes the form, étale locally on $\underline{W}$,

$$
Q \longrightarrow \mathcal{O}_{\underline{W}} \quad m \longmapsto \begin{cases}1 & m=0, \\ 0 & m \neq 0 .\end{cases}
$$

Indeed, a chart defining $\underline{W}$ must take every non-zero element of $Q$ to a function which is not invertible at any point of its domain. But as $\underline{W}$ is reduced, the only function which is non-invertible at every point of an open set of $\underline{W}$ is 0 . Thus locally $\mathcal{M}$ takes the given form.

Proposition 3.17. Let $\mathfrak{f}=(\underline{C} / \underline{W}, \mathbf{x}, \underline{f})$ be a combinatorially constant ordinary stable map over an integral, quasi-compact scheme $\underline{W}$. Then for any type $\mathbf{u}$ for $\mathfrak{f}$ the stack $\mathscr{M}(X, \mathfrak{f}, \mathbf{u})$ is quasi-compact.

Proof. Let $Q$ be the basic monoid defined by $\mathbf{u}$. Let $\mathcal{M}_{1}:=\mathcal{M}_{\underline{W}}^{0}$ be the basic $\log$ structure for the pre-stable curve $\underline{C} / \underline{W}, \mathcal{M}_{2}:=Q \times \mathcal{O}_{\underline{W}}^{\times}$the constant log structure and

$$
\bar{\varphi}: \overline{\mathcal{M}}_{1}=\prod_{q} \underline{\mathbb{N}} \longrightarrow \underline{Q}=\overline{\mathcal{M}}_{2}
$$

the homomorphism coming from the definition of $Q$. To take care of the domains of the relevant stable $\log$ maps we now look at $\underline{Y}:=\operatorname{LMor}_{\underline{W}}^{\bar{\varphi}}$ from Lemma 2.12 , By the universal property of $\mathcal{M}_{W}^{0}$ this scheme classifies log smooth structures on $\underline{C}_{\underline{V}} / \underline{V}$ with trivialized base $\log$ structure $Q \times \mathcal{O}_{\underline{V}}^{\times}$. Let $C_{\underline{Y}} / Y$ be the universal $\log$ smooth curve.

It remains to lift the pull-back $\underline{f}_{\underline{Y}}$ of $\underline{f}$ to a log morphism. This is done by $\underline{Z}:=$ $\operatorname{LMor}_{\underline{C}_{\underline{Y}} / \underline{Y}}^{\bar{\psi}}$, where now the two log structures are $\mathcal{M}_{1}:=\underline{f}_{\underline{Y}}^{*} \mathcal{M}_{X}$ and $\mathcal{M}_{2}:=\mathcal{M}_{C_{\underline{Y}}}$ on $\underline{C}_{\underline{Y}}$. The map $\bar{\psi}: \overline{\mathcal{M}}_{1} \rightarrow \overline{\mathcal{M}}_{2}$ is again fixed by basicness. We then obtain a universal basic stable $\log \operatorname{map}\left(C_{\underline{Z}} / Z, \mathbf{x}_{\underline{Z}}, f_{\underline{Z}}\right)$. Since $\underline{Z} \rightarrow \underline{W}$ is of finite type, $\underline{Z}$ is quasi-compact by quasi-compactness of $\underline{W}$.

Now the morphism $\underline{Z} \rightarrow \mathscr{M}(X, \mathfrak{f}, \mathbf{u})$ of algebraic stacks thus obtained is an epimorphism. In fact, by Lemma 3.16 locally on $\underline{W}$ the $\log$ structure $\mathcal{M}_{W}$ of a stable $\log$ map $(C / W, \mathbf{x}, f) \in \mathrm{Ob}(\mathscr{M}(X, \mathfrak{f}, \mathbf{u}))$ is isomorphic to the constant $\log$ structure $Q \times \mathcal{O}_{W}^{\times}$, and hence $(C / W, \mathbf{x}, f)$ is locally the image of the pull-back of the universal basic stable log map over $\underline{Z}$. Thus the induced map of spaces of geometric points $|\underline{Z}| \rightarrow|\mathscr{M}(X, \mathfrak{f}, \mathbf{u})|$ is a continuous surjection, and quasi-compactness of $|\mathscr{M}(X, \mathfrak{f}, \mathbf{u})|$ follows from quasi-compactness of $|\underline{Z}|$.

Lemma 3.18. Let $(\underline{C} / \underline{W}, \mathbf{x}, \underline{f})$ be an ordinary stable map with $\underline{W}$ quasi-compact. Then there exist finitely many locally closed, integral subschemes $\underline{W}_{i} \subset \underline{W}$ weakly covering $\underline{W}$ and étale surjections $\underline{\tilde{W}}_{i} \rightarrow \underline{W}_{i}$ such that the pull-back of $(\underline{C} / \underline{W}, \mathbf{x}, \underline{f})$ to $\underline{\tilde{W}}_{i}$ is combinatorially constant.

Proof. By quasi-compactness it suffices to construct the $\underline{W}_{i}$ locally. Standard arguments for families of pre-stable curves provide the $\underline{W}_{i}$ fulfilling conditions (1) and (2) in Definition 3.15. Then use the fact that $\mathcal{M}$ is a fine sheaf to also achieve (3). 
We are now in position to prove Theorem 3.12

Proof of Theorem 3.12, By the discussion at the beginning of this subsection we have to show that if $\underline{W} \rightarrow \mathscr{M}(X, \beta)$ is a morphism from a quasi-compact scheme $\underline{W}$, then $\left|\underline{W} \times_{\mathbf{M}(\underline{X})} \overline{\mathscr{M}}(X, \beta)\right|$ is quasi-compact. By the quasi-compactness criterion Lemma 3.14 together with Lemma 3.18 we can assume that $\underline{W}$ is integral and that the ordinary stable map $\mathfrak{f}=(\underline{C} / \underline{W}, \mathbf{x}, f)$ defined by $\underline{W} \rightarrow \mathbf{M}(\underline{X})$ is combinatorially constant. Let $\mathbf{u}_{1}, \ldots, \mathbf{u}_{s}$ be the finite list of types $\mathbf{u}$ of stable $\log$ maps in class $\beta$ according to Definition 3.3. From (3.2) we have the decomposition

$$
\left|\underline{W} \times_{\mathbf{M}(\underline{X})} \mathscr{M}(X, \beta)\right|=\coprod_{\mu=1}^{s}\left|\mathscr{M}\left(X, \mathfrak{f}, \mathbf{u}_{\mu}\right)\right| .
$$

Now according to Proposition 3.17 each component on the right-hand side is quasicompact. Hence $\underline{W} \times_{\mathbf{M}(\underline{X})} \mathscr{M}(X, \beta)$ is quasi-compact, as had to be shown.

\section{Stable REDUCtion}

In this section we prove a stable reduction theorem for basic stable log maps. Throughout, $R$ is a discrete valuation ring over our base scheme $\underline{S}$ with maximal ideal $\mathfrak{m}$, residue field $R / \mathfrak{m}=\kappa$ and quotient field $K$. We assume $K$ is endowed with a fine, saturated $\log$ structure over $\mathcal{M}_{S}$, hence defining a log point $\left(\operatorname{Spec} K, Q_{K}\right)$ over $S$ for a toric monoid $Q_{K}$. The closed point in $\operatorname{Spec} R$ is denoted 0 .

Theorem 4.1. Assume that $\underline{X} \rightarrow \underline{S}$ is proper. Let $\left(\pi_{K}: C_{K} \rightarrow\right.$ $\left.\left(\operatorname{Spec} K, Q_{K}\right), \mathbf{x}_{K}, f_{K}\right)$ be a basic stable log map to $X$ over the log point (Spec $\left.K, Q_{K}\right)$. Then possibly after replacing $K$ by a finite extension $\tilde{K}$ and $R$ by its integral closure in $\tilde{K}$ and pulling back the stable log map via $\left(\operatorname{Spec} \tilde{K}, Q_{K}\right) \rightarrow$ (Spec $K, Q_{K}$ ), the following holds: There exists a log structure $\mathcal{M}_{R}$ on $R$ over $S$ together with a strict morphism $\left(\operatorname{Spec} K, Q_{K}\right) \rightarrow\left(\operatorname{Spec} R, \mathcal{M}_{R}\right)$, and a basic stable log map $\left(C /\left(\operatorname{Spec} R, \mathcal{M}_{R}\right), \mathbf{x}, f\right)$ to $X$ such that the restriction to $\operatorname{Spec} K$ is isomorphic to $\left(C_{K} /\left(\operatorname{Spec} K, Q_{K}\right), \mathbf{x}_{K}, f_{K}\right)$.

Moreover, such an extension as a basic stable log map is unique up to unique isomorphism.

Corollary 4.2. For any combinatorially finite class $\beta$ of stable log maps, $\mathscr{M}(X, \beta)$ is proper over $\underline{S}$.

Proof. Recall that a morphism of algebraic stacks is proper if it is separated, of finite type and universally closed (LaMB, Definition 7.11). Separatedness follows by the uniqueness part in the theorem from the valuative criterion LaMB], Proposition 7.8. Theorem 3.12 established that $\mathscr{M}(X, \beta) \rightarrow \underline{S}$ is of finite type. In view of the existence part in Theorem 4.1 universal closedness then follows from the valuative criterion [LaMB], Theorem 7.10.

The proof of Theorem 4.1 is divided into three steps presented in the following subsections.

4.1. Extension on the level of ghost sheaves. By the stable reduction theorem for ordinary stable maps [FuPa] we may assume $\left(\underline{C}_{K} / K, \mathbf{x}_{K}, \underline{f}_{K}\right)$ is the restriction to $K$ of a marked stable map $(\underline{\pi}: \underline{C} \rightarrow$ Spec $R, \mathbf{x}, f)$ over $R$. This step may involve a base change, but note that any two such extensions are uniquely isomorphic. In particular, the central fibre $\left(\underline{C}_{0} / \operatorname{Spec} \kappa, \mathbf{x}_{0}, \underline{f}_{0}\right)$ is uniquely determined. The 
objective in this subsection is to derive the extension as a stable log map on the level of ghost sheaves. As we will see in the proof of Proposition 4.3 this amounts to determining the type of the central fibre.

The central fibre being a stable curve over a field, we use our usual convention that $\eta, q, p$ denote generic points, nodes and marked points of $\underline{C}_{0}$, respectively. For the corresponding objects on $\underline{C}_{K}$ we use an index $K$. Thus the sheaf $f^{*} \overline{\mathcal{M}}_{X}$ is determined by the usual generization maps $\chi_{\eta, p}: P_{p} \longrightarrow P_{\eta}, \chi_{\eta, q}: P_{q} \longrightarrow P_{\eta}$ on $\underline{C}_{0}$, together with the following generization maps from $\underline{C}_{0}$ to $\underline{C}_{K}$ :

$$
\chi_{\eta, K}: P_{\eta} \longrightarrow P_{\eta_{K}}, \quad \chi_{p, K}: P_{p} \longrightarrow P_{p_{K}}, \quad \chi_{q, K}: P_{q} \longrightarrow P_{q_{K}} .
$$

Note that the closures of nodes $q_{K} \in \underline{C}_{K}$ may only give a subset of the nodes of $\underline{C}_{0}$; for the other nodes of $\underline{C}_{0}$ we only have the composition $\chi_{\eta, K} \circ \chi_{\eta, q}: P_{q} \rightarrow P_{\eta_{K}}$. Let us refer to the latter as isolated nodes.

Proposition 4.3. There exists a type $\mathbf{u}=\left(u_{x}\right)_{x \in \underline{C}_{0}}$ for stable log maps with underlying ordinary stable map $\left(\underline{C}_{0} / \operatorname{Spec} \kappa, \mathbf{x}_{0}, \underline{f}_{0}\right)$ such that the central fibre of any extension to $\operatorname{Spec} R$ of $\left(C_{K} /\left(\operatorname{Spec} K, Q_{K}\right), \mathbf{x}_{K}, f_{K}\right)$ as a basic stable log map has type $\mathbf{u}$. Moreover, $\mathbf{u}$ defines the extension on the level of ghost sheaves uniquely up to unique isomorphism.

Proof. Let $\left(C /\left(\operatorname{Spec} R, \mathcal{M}_{R}\right), \mathbf{x}, f\right)$ be an extension of $\left(C_{K} /\left(\operatorname{Spec} K, Q_{K}\right), \mathbf{x}_{K}, f_{K}\right)$. We are going to show that this extension is determined uniquely on the level of ghost sheaves by data computable without knowing the extension.

By basicness we have a commutative diagram

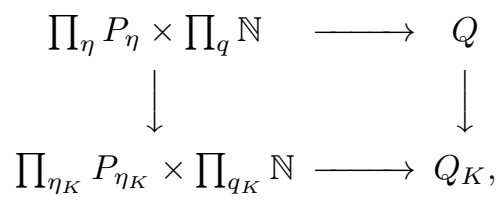

defining the basic monoids $Q, Q_{K}$ for the central and the generic fibres, respectively. The left-hand vertical arrow in the diagram is defined as the product of $\prod \chi_{\eta, K}$ and the projection to the non-isolated nodes. The horizontal arrows are quotients by subgroups $R_{\mathbf{u}} \subset \prod_{\eta} P_{\eta}^{\mathrm{gp}} \times \prod_{q} \mathbb{Z}$ and $R_{\mathbf{u}_{K}} \subset \prod_{\eta_{K}} P_{\eta_{K}}^{\mathrm{gp}} \times \prod_{q_{K}} \mathbb{Z}$, determined by the respective types $\mathbf{u}$ and $\mathbf{u}_{K}$, followed by saturation; see Construction 1.16 .

Recall also from Discussion 1.8 that the upper horizontal map together with the types $u_{p}$ of the marked points determine the maps of ghost sheaves $\pi^{*} \overline{\mathcal{M}}_{R} \rightarrow \overline{\mathcal{M}}_{C}$, $f^{*} \overline{\mathcal{M}}_{X} \rightarrow \overline{\mathcal{M}}_{C}$ on the central fibre, and similarly for the lower horizontal arrow and the generic fibre. Commutativity of the diagram then establishes the generization maps between the relevant sheaves, and hence determines the extension on the level of ghost sheaves. Thus it suffices to find the type $\mathbf{u}=\left\{\left(u_{p}\right)_{p},\left(u_{q}\right)_{q}\right\}$ with $R_{\mathbf{u}}$ mapping to $R_{\mathbf{u}_{K}}$.

If $p \in \underline{C}_{0}$ is a marked point there exists a unique marked point $p_{K} \in \underline{C}_{K}$ with $p \in \operatorname{cl}\left(p_{K}\right)$. By the structure of $\log$ curves (Theorem 1.1(ii)) $u_{p}$ must then equal the composition

$$
P_{p} \stackrel{\chi_{p, K}}{\longrightarrow} P_{p_{K}} \stackrel{u_{p_{K}}}{\longrightarrow} \mathbb{N},
$$

where the second map is given by the type of the generic fibre. Hence $u_{p}$ depends only on the generic fibre and $\underline{f}$. 
Similarly, for a non-isolated node $q$ let $q_{K}$ be the node of $\underline{C}_{K}$ with $q \in \operatorname{cl}\left(q_{K}\right)$. Then the defining equation (1.8) for $u_{q}$,

$$
\varphi_{\bar{\eta}_{2}}\left(\chi_{\eta_{2}, q}(m)\right)-\varphi_{\bar{\eta}_{1}}\left(\chi_{\eta_{1}, q}(m)\right)=u_{q}(m) \cdot \rho_{q},
$$

with $\varphi:=\overline{f^{b}}$ and $m \in P_{q}$, generizes to the defining equation for $u_{q_{K}}$ :

$$
\varphi_{\bar{\eta}_{2, K}}\left(\chi_{\eta_{2, K}, q_{K}}\left(m_{K}\right)\right)-\varphi_{\bar{\eta}_{1, K}}\left(\chi_{\eta_{1, K}, q_{K}}\left(m_{K}\right)\right)=u_{q_{K}}\left(m_{K}\right) \cdot \rho_{q_{K}},
$$

where $m_{K}:=\chi_{q, K}(m)$. Hence $u_{q}=u_{q_{K}} \circ \chi_{q, K}$ is also determined a priori by the generic fibre.

So far we have just reversed the reasoning in the proof of Proposition 1.22. The isolated nodes, however, require different arguments. Recall that $u_{q}$ was defined by the homomorphism

$$
\varphi_{\bar{q}}: P_{q}=\overline{\mathcal{M}}_{X, \underline{f}(q)} \longrightarrow \overline{\mathcal{M}}_{C, \bar{q}}=Q \oplus_{\mathbb{N}} \mathbb{N}^{2}, \quad m \longmapsto\left(m^{\prime},(a, b)\right)
$$

as $u_{q}(m)=b-a$ (for the correct orientation). Now while the composition with generization to $\eta_{K}$ maps any $(0,(a, b))$ to $0 \in Q_{K}$, we can retrieve $b-a$ by working on the level of log structures. In fact, choosing a compatible chart on an étale neighbourhood of $q$, the generization map has the form

$$
\begin{aligned}
\left(Q \oplus_{\mathbb{N}} \mathbb{N}^{2}\right) \times \mathcal{O}_{\underline{C}, \bar{q}}^{\times} \simeq \mathcal{M}_{C, \bar{q}} & \longrightarrow \mathcal{M}_{C, \bar{\eta}_{K}} \simeq Q_{K} \times \mathcal{O}_{\underline{\underline{C}}, \bar{\eta}_{K}}^{\times} \\
\left(\left(m^{\prime},(a, b)\right), h\right) & \longmapsto\left(m_{K}^{\prime}, z^{a} w^{b} \underline{\pi}^{*} g\left(m^{\prime}\right) h\right),
\end{aligned}
$$

with $z=0, w=0$ defining the two branches of $\underline{C}_{0}$ inside $\underline{C}$ at $q$, and $g\left(m^{\prime}\right) \in K^{\times}$. Assume that $\underline{C}$ has an $A_{e-1}$-singularity at $q$. Then letting $\operatorname{ord}_{\eta_{1}}, \operatorname{ord}_{\eta_{2}}$ be the discrete valuations of these branches it holds $\operatorname{ord}_{\eta_{1}}(z)=\operatorname{ord}_{\eta_{2}}(w)=e$, and hence $e \cdot(b-a)=\left(\operatorname{ord}_{\eta_{2}}-\operatorname{ord}_{\eta_{1}}\right)\left(z^{a} w^{b} h\right)$ and $\operatorname{ord}_{\eta_{1}}\left(\underline{\pi}^{*}\left(g\left(m^{\prime}\right)\right)\right)=\operatorname{ord}_{\eta_{2}}\left(\underline{\pi}^{*}\left(g\left(m^{\prime}\right)\right)\right)$. Thus $e \cdot u_{q}$ equals the following composition:

$$
P_{q} \longrightarrow \mathcal{M}_{X, \underline{f}(q)} \stackrel{f^{b}}{\longrightarrow} \mathcal{M}_{C, \bar{q}} \longrightarrow \mathcal{M}_{C, \bar{\eta}_{K}} \simeq Q_{K} \times \mathcal{O}_{\underline{C}, \bar{\eta}_{K}}^{\times} \stackrel{\mathrm{pr}_{2}}{\longrightarrow} \mathcal{O}_{\underline{C}, \bar{\eta}_{K}}^{\times} \stackrel{\operatorname{ord}_{\eta_{2}}-\operatorname{ord}_{\eta_{1}}}{\longrightarrow} \mathbb{Z} .
$$

The first map is a choice of chart for $\underline{f}^{*} \mathcal{M}_{X}$ at $q$. Finally, observe that the composition

equals

$$
\mathcal{M}_{X, \underline{f}(q)} \rightarrow \mathcal{M}_{C, \bar{q}} \rightarrow \mathcal{M}_{C, \bar{\eta}_{K}}
$$

$$
\mathcal{M}_{X, \underline{f}(q)} \rightarrow \mathcal{M}_{X, \underline{f}\left(\eta_{K}\right)} \stackrel{f_{K}^{b}}{\longrightarrow} \mathcal{M}_{C, \bar{\eta}_{K}} .
$$

Hence $u_{q}$ is completely determined also for an isolated node $q$ by the generic fibre and by the extension $\underline{f}$ of $\underline{f}_{K}$, already known to be unique.

4.2. The log structure on the base. We now want to show that the extension of the $\log$ structure on the base is uniquely defined. The decisive tool is the identification of "fibrewise constant" subsheaves $\mathcal{M}(\eta)$ of $f^{*} \mathcal{M}_{X}$ on certain open subsets of $\underline{C}$ as follows. There is one open subset $U(\eta)$ for each generic point $\eta \in \underline{C}_{0}$. To define $U(\eta)$ let $A \subset \underline{C}_{0}$ be the set of non-special points of $\operatorname{cl}(\eta) \subset \underline{C}_{0}$. Then $U(\eta) \subset \underline{C}$ is the set of generizations of points in $A$. Said differently, if $D \subset \underline{C}$ is the irreducible component of $\underline{C}$ containing $\eta$, then $U(\eta)$ is obtained from $D$ by removing all special points and all irreducible components of $\underline{C}_{0}$ not containing $\eta$. Thus $U(\eta)$ is a smooth open neighbourhood of $\eta$, and $\left.\underline{f}^{*} \overline{\mathcal{M}}_{X}\right|_{U(\eta)}$ has only two interesting stalks, at $\eta$ and at its generization $\eta_{K}$; see Remark 1.9. Hence $\left.\underline{f}^{*} \overline{\mathcal{M}}_{X}\right|_{U(\eta)}$ is completely 
determined by the generization map $P_{\eta} \rightarrow P_{\eta_{K}}$. In particular, $\left.f^{*} \overline{\mathcal{M}}_{X}\right|_{U(\eta)}$ is globally generated with global section space equal to $P_{\eta}$. Note also that each $x \in U(\eta)$ is in the closure of $\eta_{K}$, so we may restrict germs of sections to $\eta_{K}$. The notation for the restriction of $m$ is $\left.m\right|_{\eta_{K}}$.

Definition 4.4. The fibrewise constant subsheaf $\left.\mathcal{M}(\eta) \subset f^{*} \mathcal{M}_{X}\right|_{U(\eta)}$ is the sheaf in the Zariski topology defined by the condition

$$
m \in \mathcal{M}(\eta)_{x} \Longleftrightarrow f_{K}^{b}\left(\left.m\right|_{\eta_{K}}\right) \in \operatorname{im}\left(\Gamma\left(\mathcal{M}_{K}\right) \stackrel{\pi_{K}^{b}}{\longrightarrow} \mathcal{M}_{C, \bar{\eta}_{K}}\right) .
$$

Since $C_{K} \rightarrow\left(\operatorname{Spec} K, Q_{K}\right)$ is strict at $\bar{\eta}_{K}$, the map $\pi_{K}^{b}$ is injective and there is a canonical map

$$
\beta_{\eta}: \mathcal{M}(\eta)_{\eta} \longrightarrow \Gamma\left(\mathcal{M}_{K}\right), \quad m \longmapsto\left(\pi_{K}^{b}\right)^{-1}\left(f_{K}^{b}\left(\left.m\right|_{\eta_{K}}\right)\right) .
$$

Note the composition of $\beta_{\eta}$ with the structure homomorphism $\Gamma\left(\mathcal{M}_{K}\right) \rightarrow K$ factors over a map

$$
\alpha_{\eta}: \mathcal{M}(\eta)_{\eta} \longrightarrow R
$$

In fact, if $m \in \mathcal{M}(\eta)_{\eta}$ maps to $a \in K$, then $\pi^{*}(a)$ is in the image of the structure homomorphism $\mathcal{M}(\eta)_{\eta} \rightarrow \mathcal{O}_{\underline{C}, \eta}$ and hence $a$ is regular at 0 .

Calling $\mathcal{M}(\eta)$ fibrewise constant is justified by the fact that the restrictions of $\mathcal{M}(\eta)$ to $\underline{C}_{K} \cap U(\eta)$ and to $\underline{C}_{0} \cap U(\eta)$ are constant sheaves with fibres isomorphic to $P_{\eta_{K}} \times K^{\times}$and to $P_{\eta} \times R^{\times}$, respectively. For our purposes only the stalk $\mathcal{M}(\eta)_{\eta}$ is really relevant and it suffices to prove the following weaker statement.

Lemma 4.5. There is a (non-canonical) isomorphism $\mathcal{M}(\eta)_{\eta} \simeq P_{\eta} \times R^{\times}$.

Proof. By strictness of $C_{K} \rightarrow\left(\operatorname{Spec} K, Q_{K}\right)$ at $\bar{\eta}_{K}$ there exists an isomorphism $\mathcal{M}_{C, \bar{\eta}_{K}} \simeq Q_{K} \times \mathcal{O}_{\underline{C}, \bar{\eta}_{K}}^{\times}$such that $\pi_{K}^{b}\left(\Gamma\left(\mathcal{M}_{K}\right)\right)=Q_{K} \times K^{\times}$. Let $\theta: P_{\eta} \rightarrow \mathcal{M}_{X, \underline{f}(\eta)}$ be a chart, hence inducing an isomorphism $\left(f^{*} \mathcal{M}_{X}\right)_{\eta} \simeq P_{\eta} \times \mathcal{O}_{\underline{C}, \eta}^{\times}$. Note that $\mathcal{O}_{\underline{C}, \bar{\eta}_{K}}^{\times} \simeq \mathbb{Z} \times \mathcal{O}_{\underline{C}, \bar{\eta}}^{\times}$since $R$ is a discrete valuation ring. We can thus define $g: P_{\eta} \rightarrow$ $\mathcal{O}_{\underline{\underline{C}}, \bar{\eta}}^{\bar{x}}$ by the composition

$P_{\eta} \stackrel{\theta}{\longrightarrow} \mathcal{M}_{X, \underline{f}(\eta)} \stackrel{f^{b}\left(\left.\right|_{\eta_{K}}\right)}{\longrightarrow} \mathcal{M}_{C_{K}, \bar{\eta}_{K}} \simeq Q_{K} \times \mathcal{O}_{\underline{C}, \bar{\eta}_{K}}^{\times} \stackrel{\mathrm{pr}_{2}}{\longrightarrow} \mathcal{O}_{\underline{C}, \bar{\eta}_{K}}^{\times} \simeq \mathbb{Z} \times \mathcal{O}_{\underline{C}, \bar{\eta}}^{\times} \stackrel{\mathrm{pr}_{2}}{\longrightarrow} \mathcal{O}_{\underline{C}, \bar{\eta}}^{\times}$.

Note that $g(m)$ measures the defect of $\theta(m)$ to be fibrewise constant. Thus

$$
g^{-1} \cdot \theta: P_{\eta} \longrightarrow \mathcal{M}_{X, \underline{f}(\eta)}, \quad p \longmapsto g^{-1}(p) \cdot \theta(p)
$$

factors over $\mathcal{M}(\eta)_{\eta}$. From the analogous property of $\theta$ the constructed homomorphism $P_{\eta} \rightarrow \mathcal{M}(\eta)_{\eta}$ is right-inverse to

$$
\mathcal{M}(\eta)_{\eta} \rightarrow \mathcal{M}_{X, \underline{f}(\eta)} \rightarrow \overline{\mathcal{M}}_{X, \underline{f}(\eta)}=P_{\eta}, \quad m \longmapsto \bar{m} .
$$

To prove the conclusion it remains to show that if $m_{1}, m_{2} \in \mathcal{M}(\eta)_{\eta}$ fulfill $\bar{m}_{1}=\bar{m}_{2}$ there exists a unique $h \in R^{\times}$with $m_{2}=h \cdot m_{1}$. By the definition of the ghost sheaf there certainly exists $h \in \mathcal{O}_{\underline{C}, \eta}^{\times}$such that $m_{2}=h \cdot m_{1}$ holds in $\mathcal{M}_{X, \underline{f}(\eta)}$. But then also $f_{K}^{b}\left(\left.m_{2}\right|_{\eta_{K}}\right)=h \cdot f_{K}^{b}\left(\left.m_{1}\right|_{\eta_{K}}\right)$ holds in $\mathcal{M}_{C, \bar{\eta}_{K}}$. Since both $m_{1}, m_{2}$ are fibrewise constant it follows $h \in K^{\times}$. Hence $h \in K^{\times} \cap \mathcal{O}_{\underline{C}, \eta}^{\times}=R^{\times}$as claimed.

To motivate our construction of the $\log$ structure on $R$ note that given $\mathcal{M}_{R}$, the product

$$
\mathcal{M}_{R, 0} \longrightarrow Q \times_{Q_{K}} \Gamma\left(\mathcal{M}_{K}\right)
$$


of the quotient $\mathcal{M}_{R, 0} \rightarrow \overline{\mathcal{M}}_{R, 0}=Q$ and the generization map exhibits $\mathcal{M}_{R, 0}$ canonically as a submonoid of $Q \times{ }_{Q_{K}} \Gamma\left(\mathcal{M}_{K}\right)$. The point is that we can determine this image without knowledge of $\mathcal{M}_{R, 0}$. To this end let $\alpha_{0}: \mathcal{M}_{R}^{0} \rightarrow \mathcal{O}_{\text {Spec } R}$ be the basic $\log$ structure on $\operatorname{Spec} R$ for the pre-stable curve $(\underline{C} / \operatorname{Spec} R, \mathbf{x})$. The universal property of this log structure produces a homomorphism

$$
\beta_{0}: \mathcal{M}_{R, 0}^{0} \longrightarrow \mathcal{M}_{R,(0)}^{0} \longrightarrow \Gamma\left(\mathcal{M}_{K}\right)
$$

Here $(0) \in \operatorname{Spec} R$ denotes the generic point. From the definition of $Q$ there are also canonical homomorphisms

$$
\psi_{\eta}: \mathcal{M}(\eta)_{\eta} \longrightarrow P_{\eta} \longrightarrow Q, \quad \psi_{0}: \mathcal{M}_{R, 0}^{0} \longrightarrow \prod_{q} \mathbb{N} \longrightarrow Q .
$$

Define $\beta:=\left(\prod_{\eta} \beta_{\eta}\right) \cdot \beta_{0}$ and $\psi:=\left(\sum_{\eta} \psi_{\eta}\right)+\psi_{0}$. Clearly, $\psi_{\eta} \times \beta_{\eta}$ and $\psi_{0} \times \beta_{0}$ have image in $Q \times{ }_{Q_{K}} \Gamma\left(\mathcal{M}_{K}\right) \subset Q \times \Gamma\left(\mathcal{M}_{K}\right)$. Thus $\psi \times \beta$ maps $\left(\prod_{\eta} \mathcal{M}(\eta)_{\eta}\right) \times \mathcal{M}_{R, 0}^{0}$ to $Q \times{ }_{Q_{K}} \Gamma\left(\mathcal{M}_{K}\right)$. We are now in position to construct $\mathcal{M}_{R}$ canonically.

Construction 4.6. Let $\mathcal{M}_{R}^{\prime}$ be the log structure on $R$ extending $\mathcal{M}_{K}$ by the stalk

$$
\mathcal{M}_{R, 0}^{\prime}:=\operatorname{im}(\psi \times \beta) \subset Q \times{ }_{Q_{K}} \Gamma\left(\mathcal{M}_{K}\right)
$$

at 0 and by the projection to $\Gamma\left(\mathcal{M}_{K}\right)$ as the generization map to the generic point. Note that $\mathcal{M}_{R, 0}^{\prime}$ may not be saturated, so we now take its saturation to define $\mathcal{M}_{R, 0}$. Since $Q$ and $\Gamma\left(\mathcal{M}_{K}\right)$ are saturated, so is $Q \times{ }_{Q_{K}} \Gamma\left(\mathcal{M}_{K}\right)$. Thus $\mathcal{M}_{R, 0}$ is still canonically a submonoid of $Q \times{ }_{Q_{K}} \Gamma\left(\mathcal{M}_{K}\right)$. For the structure homomorphism $\alpha_{R, 0}$ : $\mathcal{M}_{R, 0} \rightarrow R$ to be compatible with generization it must be taken as composition

$$
\mathcal{M}_{R, 0} \longrightarrow Q \times_{Q_{K}} \Gamma\left(\mathcal{M}_{K}\right) \stackrel{\mathrm{pr}_{2}}{\longrightarrow} \Gamma\left(\mathcal{M}_{K}\right) \longrightarrow K
$$

which we claim has image in $R$. In fact, since $a \in K$ lies in $R$ iff $a^{d} \in R$ for some $d>0$ it suffices to check this statement before saturation. But the restriction of (4.4) to $\mathcal{M}_{R, 0}^{\prime}$ is compatible with $\left(\prod_{\eta} \alpha_{\eta}\right) \times \alpha_{0}$ and hence indeed has image in $R$.

Here is the key technical result for establishing that $\mathcal{M}_{R}$ has the requested properties.

Lemma 4.7. $\beta^{\mathrm{gp}}\left(\operatorname{ker}\left(\psi^{\mathrm{gp}}\right)\right)=R^{\times}$, where $R^{\times} \subset \Gamma\left(\mathcal{M}_{K}\right)$ is canonically embedded via the inverse of the structure homomorphism $\Gamma\left(\mathcal{M}_{K}\right) \rightarrow K$.

Proof. Each $\beta_{\eta}$ as well as $\beta_{0}$ are equivariant with respect to the multiplication action of $R^{\times}$, while $\psi_{\eta}$ and $\psi_{0}$ are invariant under this action. Since $1 \in R^{\times}$lies in the image it is therefore enough to show $\beta^{\mathrm{gp}}\left(\operatorname{ker}\left(\psi^{\mathrm{gp}}\right)\right) \subset R^{\times}$.

An element $m=\left(\left(m_{\eta}\right)_{\eta}, m_{0}\right) \in\left(\prod_{\eta} \mathcal{M}(\eta)_{\eta}^{\mathrm{gp}}\right) \times\left(\mathcal{M}_{R, 0}^{0}\right)^{\mathrm{gp}}$ lies in ker $\psi^{\mathrm{gp}}$ iff $\bar{m} \in \prod_{\eta} P_{\eta}^{\mathrm{gp}} \times \prod_{q} \mathbb{Z}$ lies in the relation subgroup $R_{\mathbf{u}}$ defining $Q$. In particular, $\psi(\bar{m})$ generizes to $0 \in Q_{K}$, and hence $\beta^{\mathrm{gp}}\left(\operatorname{ker}\left(\psi^{\mathrm{gp}}\right)\right) \subset K^{\times}$. It also suffices to check the statement for $\bar{m}$ equal to one of the generators $a_{q}\left(\bar{m}_{q}\right), \bar{m}_{q} \in P_{q}$, of $R_{\mathbf{u}}$, as defined in Construction 1.16. Note this holds regardless of saturation since $a \in K^{\times}$lies in $R^{\times}$iff $a^{d} \in R^{\times}$for some $d>0$. Thus we have to show that if $m=\left(\left(m_{\eta}\right)_{\eta}, m_{0}\right) \in$ $\left(\prod_{\eta} \mathcal{M}(\eta)_{\eta}^{\mathrm{gp}}\right) \times\left(\mathcal{M}_{R, 0}^{0}\right)^{\mathrm{gp}}$ maps to some $a_{q}\left(\bar{m}_{q}\right)$, then $\operatorname{ord}_{0}\left(\beta^{\mathrm{gp}}(m)\right)=0$, where $\operatorname{ord}_{0}$ is the discrete valuation of $K$. Note from the definition of $a_{q}\left(\bar{m}_{q}\right)$ that $\beta_{\eta}\left(m_{\eta}\right) \in$ $R^{\times}$except possibly for $\eta=\eta_{1}, \eta_{2}$, the generic points of the branches of $\underline{C}_{0}$ at $q$. Similarly, all entries of $\bar{m}_{0} \in \prod_{q} \mathbb{Z}$ vanish except at entry $q$, which equals $u_{q}\left(\bar{m}_{q}\right)$. By working on an étale neighbourhood of $q$ we may assume $\underline{C} \rightarrow \operatorname{Spec} R$ is locally given by $\operatorname{Spec}\left(R[z, w] /\left(z w-t_{q}\right)\right)$ with $t_{q} \in R$. Let $m_{q} \in \mathcal{M}_{X, f(q)}$ be a lift of $\bar{m}_{q}$. 
There are two cases, depending on $q$ being an isolated node or not. Let us first assume $q$ is isolated. Then $t_{q} \neq 0$ and $\beta_{0}\left(m_{0}\right)=h \cdot t_{q}^{u_{q}\left(\bar{m}_{q}\right)}$ for some $h \in$ $R^{\times}$. Denote by $\eta_{K} \in \underline{C}_{K}$ the generic point with $\eta_{1}, \eta_{2} \in \operatorname{cl}\left(\eta_{K}\right)$ as before. Let $\mathcal{M}_{C_{K}, \bar{\eta}_{K}} \simeq Q_{K} \times \mathcal{O}_{\underline{C}, \bar{\eta}_{K}}^{\times}$be an isomorphism induced by a distinguished chart as in Theorem 1.1. In particular, $\pi_{K}^{b}: \Gamma\left(\mathcal{M}_{K}\right) \rightarrow \mathcal{M}_{C_{K}, \bar{\eta}_{K}}$ is then given by the natural inclusion $Q_{K} \times K^{\times} \rightarrow Q_{K} \times \mathcal{O}_{\underline{C}_{K}, \bar{\eta}_{K}}^{\times}$. As in (4.1) consider the composition

$$
\kappa_{q}: \mathcal{M}_{X, \underline{f}(q)} \longrightarrow \mathcal{M}_{X, \underline{f}\left(\eta_{K}\right)} \stackrel{f_{K}^{b}}{\longrightarrow} \mathcal{M}_{C_{K}, \bar{\eta}_{K}} \simeq Q_{K} \times \mathcal{O}_{\underline{\underline{C}}, \bar{\eta}_{K}}^{\times} \stackrel{\mathrm{pr}_{2}}{\longrightarrow} \mathcal{O}_{\underline{\underline{C}}, \bar{\eta}_{K}}^{\times} .
$$

Since $\bar{m}_{q}$ generizes to $\bar{m}_{\eta_{1}}$ and to $-\bar{m}_{\eta_{2}}$, respectively, the generizations of $m_{q}$ at $\eta_{1}, \eta_{2}$ differ from $m_{\eta_{1}}$ and $m_{\eta_{2}}^{-1}$ only by functions invertible at $\eta_{i}$. Therefore

$$
\begin{aligned}
\operatorname{ord}_{0}(\beta(m)) & =\operatorname{ord}_{0}\left(\beta_{\eta_{1}}\left(m_{\eta_{1}}\right) \cdot \beta_{\eta_{2}}\left(m_{\eta_{2}}\right) \cdot \beta_{0}\left(m_{0}\right)\right) \\
& =\operatorname{ord}_{\eta_{1}}\left(\kappa_{q}\left(m_{q}\right)\right)-\operatorname{ord}_{\eta_{2}}\left(\kappa_{q}\left(m_{q}\right)\right)+\operatorname{ord}_{0}\left(t_{q}^{u_{q}\left(\bar{m}_{q}\right)}\right) .
\end{aligned}
$$

Now by the discussion of the map (4.1) at the end of $\$ 4.1$ we know

$$
e \cdot u_{q}\left(\bar{m}_{q}\right)=\operatorname{ord}_{\eta_{2}}\left(\kappa_{q}\left(m_{q}\right)\right)-\operatorname{ord}_{\eta_{1}}\left(\kappa_{q}\left(m_{q}\right)\right)
$$

and $e=\operatorname{ord}_{0}\left(t_{q}\right)$. Thus $\operatorname{ord}_{0}(\beta(m))=0$ as claimed. Note that the argument seems to depend on the choice of chart inducing the isomorphism $\mathcal{M}_{C_{K}, \bar{\eta}_{K}} \simeq Q_{K} \times \mathcal{O}_{\underline{C}, \bar{\eta}_{K}}^{\times}$, but different choices cancel out in the formula for $\operatorname{ord}_{0}(\beta(m))$ due to the different signs for $\eta_{1}$ and $\eta_{2}$.

For a non-isolated node $q$ let $q_{K}$ be the node of $\underline{C}_{K}$ with $q \in \operatorname{cl}\left(q_{K}\right)$. Now $t_{q}=0$ and a distinguished chart for $C_{K}$ at $q_{K}$ takes the form

$$
\mathcal{M}_{C_{K}, \bar{q}_{K}} \simeq\left(Q_{K} \oplus_{\mathbb{N}} \mathbb{N}^{2}\right) \times \mathcal{O}_{\underline{C}_{K}, \bar{q}_{K}}^{\times} .
$$

Again denote by $\kappa_{q}: \mathcal{M}_{X, \underline{f}(q)} \rightarrow \mathcal{O}_{\underline{C}_{K}, \bar{q}_{K}}^{\times}$the composition of $f^{b}\left(\left.\cdot\right|_{q_{K}}\right)$ with the projection induced by the chart, and similarly let $\kappa_{K}: \Gamma\left(\mathcal{M}_{K}\right)=Q_{K} \times K^{\times} \rightarrow K^{\times}$ be the projection. Since $\bar{m}=a_{q}\left(\bar{m}_{q}\right)$ the generization of $\bar{m}_{q}$ to $\eta_{i}$ is $\pm \bar{m}_{\eta_{i}}$. Hence the generization of $m_{q}$ to $\eta_{i}$ is $m_{\eta_{i}}^{ \pm 1}$ as element of $\mathcal{M}_{X, \underline{f}\left(\eta_{i}\right)}^{\mathrm{gp}}$ up to an element of $\mathcal{O}_{\underline{C}, \eta_{i}}^{\times}$. Since $\kappa_{K}$ is induced from $\kappa_{q}$ and by the definition of $\beta_{\eta_{i}}$, the elements $\pi_{K}^{*}\left(\kappa_{K}\left(\beta_{\eta_{i}}\left(m_{\eta_{i}}^{ \pm 1}\right)\right)\right)$ and $\left.\kappa_{q}\left(m_{q}\right)\right|_{\bar{\eta}_{K}}$ of $\mathcal{O}_{\underline{C}, \bar{\eta}_{K}}^{\times}$agree up to an element of $\mathcal{O}_{\underline{C}, \bar{\eta}_{i}}^{\times}$. Therefore,

$$
\pm \operatorname{ord}_{0}\left(\kappa_{K}\left(\beta_{\eta_{i}}\left(m_{\eta_{i}}\right)\right)\right)=\operatorname{ord}_{\eta_{i}}\left(\kappa_{q}\left(m_{q}\right)\right)=\operatorname{ord}_{\eta_{i}}(h),
$$

with $h:=\kappa_{q}\left(m_{q}\right) \in \mathcal{O}_{\underline{C}_{K}, \bar{q}_{K}}^{\times}$and opposite signs for $i=1,2$. Now $h$ is invertible at $\bar{q}_{K}$ and, since $\kappa_{q}$ factors over a chart for $\mathcal{M}_{C_{K}}$, the zero and polar locus of $h$ is contained in $\underline{C}_{0}$. Hence we can write $h=g_{q} g_{K}$ with $g_{q} \in \mathcal{O}_{\underline{C}, \bar{q}}^{\times}, g_{K} \in K^{\times}$, and thus $\operatorname{ord}_{\eta_{1}}(h)=\operatorname{ord}_{0}\left(g_{K}\right)=\operatorname{ord}_{\eta_{2}}(h)$. This shows

$$
\kappa_{K}\left(\beta_{\eta_{1}}\left(m_{\eta_{1}}\right) \cdot \beta_{\eta_{2}}\left(m_{\eta_{2}}\right)\right) \in R^{\times} .
$$

As for $m_{0}$ note that there is a lift to $\mathcal{M}_{R, 0}^{0}$ of the $q$-th generator of $\overline{\mathcal{M}}_{R, 0}^{0}=\prod_{q} \mathbb{N}$ mapping to $(0,(1,1), 1) \in\left(Q_{K} \oplus_{\mathbb{N}} \mathbb{N}^{2}\right) \times \mathcal{O}_{\underline{C}_{K}, \bar{q}_{K}}^{\times}$in our chart. Hence in our chart $\pi_{K}^{b}\left(\beta_{0}\left(m_{0}\right)\right)$ takes the form $((0,(a, a)), h)$ for $a=u_{q}\left(\bar{m}_{q}\right) \in \mathbb{N} \backslash\{0\}$ and $h \in \mathcal{O}_{\underline{C}, \bar{q}}^{\times}$. Therefore also $\kappa_{K}\left(\beta_{0}\left(m_{0}\right)\right) \in R^{\times}$. Taken together we obtain $\beta(m) \in R^{\times}$as claimed. The independence of this argument of the choice of charts follows as in the case of isolated nodes. 
Proposition 4.8. The extension $\alpha_{R}: \mathcal{M}_{R} \rightarrow \mathcal{O}_{\text {Spec } R}$ of $\mathcal{M}_{K}$ defined by $\mathcal{M}_{R, 0} \subset$ $Q \times{ }_{Q_{K}} \Gamma\left(\mathcal{M}_{K}\right)$ (Construction 4.6) is a log structure on $R$ with $\overline{\mathcal{M}}_{R, 0}=Q$. Moreover, if $\mathcal{M}_{R}^{\prime}$ is the log structure on $\operatorname{Spec} R$ for an extension of $\left(C_{K} /\left(\operatorname{Spec} K, Q_{K}\right)\right.$, $\left.\mathbf{x}_{K}, f_{K}\right)$ to a basic stable log map over $R$, then the image of $\mathcal{M}_{R, 0}^{\prime}$ under the canonical map to $Q \times{ }_{Q_{K}} \Gamma\left(\mathcal{M}_{K}\right)$ equals $\mathcal{M}_{R, 0}$.

Proof. By Lemma 4.5 the maps $\mathcal{M}(\eta)_{\eta} \rightarrow \overline{\mathcal{M}}_{X, f(\eta)}=P_{\eta}$ are surjective, as is $\mathcal{M}_{R, 0}^{0} \rightarrow \prod_{q} \mathbb{N}$. Hence $\psi:\left(\prod_{\eta} \mathcal{M}(\eta)_{\eta}\right) \times \mathcal{M}_{R, 0}^{0} \longrightarrow Q$ is surjective up to saturation by the definition of $Q$. Since $\mathcal{M}_{R, 0}=\operatorname{im}(\psi \times \beta)^{\text {sat }}$ the projection to the first factor

$$
\kappa: \mathcal{M}_{R, 0} \longrightarrow Q \times{ }_{Q_{K}} \Gamma\left(\mathcal{M}_{K}\right) \longrightarrow Q
$$

is also surjective up to saturation. Since $\kappa$ is also $R^{\times}$-invariant we obtain a homomorphism $\mathcal{M}_{R, 0} / R^{\times} \rightarrow Q$ that is surjective up to saturation. Conversely, if $m \in \mathcal{M}_{R, 0}$ maps to a non-zero element in $Q$, then $\alpha_{R, 0}(m) \in R$ is not invertible. Indeed, let $\left(\left(m_{\eta}\right), m_{0}\right) \in \prod_{\eta} \mathcal{M}(\eta)_{\eta} \times \mathcal{M}_{R, 0}^{0}$ be a lift of (a power of) $m$. Then by the definition of $\psi$ we must have $\psi_{0}\left(m_{0}\right) \neq 0$ or $\psi_{\eta}\left(m_{\eta}\right) \neq 0$ for some $\eta$. But since $\alpha_{\eta}$ and $\alpha_{0}$ are induced by charts of log structures, one of $\alpha_{0}\left(m_{0}\right)$ or $\alpha_{\eta}\left(m_{\eta}\right)$ is not invertible. Hence also $\alpha_{R, 0}(m)$ is not invertible. This shows $\alpha_{R, 0}^{-1}\left(R^{\times}\right) \subset \mathcal{M}_{R, 0} \cap\left(\{0\} \times \Gamma\left(\mathcal{M}_{K}\right)\right)$. Thus Lemma 4.7 says that $\alpha_{R, 0}$ maps $\alpha_{R, 0}^{-1}\left(R^{\times}\right)$isomorphically to $R^{\times} \subset K^{\times}$. In particular, $\alpha_{R, 0}$ induces an injection of $\mathcal{M}_{R, 0} / R^{\times}$into $Q$ that is surjective up to saturation. Since both $\mathcal{M}_{R, 0}$ and $Q$ are saturated, this map is an isomorphism. We have thus established that $\mathcal{M}_{R} \rightarrow \mathcal{O}_{R}$ is a $\log$ structure on $R$ with $\overline{\mathcal{M}}_{R, 0}=Q$.

For the uniqueness statement it suffices to produce a factorization of $\psi \times \beta$ over $\mathcal{M}_{R, 0}^{\prime}$ :

$$
\left(\prod_{\eta} \mathcal{M}(\eta)_{\eta}\right) \times \mathcal{M}_{R, 0}^{0} \longrightarrow \mathcal{M}_{R, 0}^{\prime} \longrightarrow Q \times_{Q_{K}} \Gamma\left(\mathcal{M}_{K}\right) .
$$

In fact, then $\mathcal{M}_{R, 0} \subset \operatorname{im}\left(\mathcal{M}_{R, 0}^{\prime} \rightarrow Q \times_{Q_{K}} \Gamma\left(\mathcal{M}_{K}\right)\right)$ and equality in this inclusion follows by the equality of ghost sheaves via basicness. The factorization is immediate on $\mathcal{M}_{R, 0}^{0}$ by the universal property of this log structure. For $\mathcal{M}(\eta)_{\eta}$ recall first that $\Gamma\left(U(\eta), \underline{f}^{*} \overline{\mathcal{M}}_{X}\right)=P_{\eta}$. Thus for each $\bar{m} \in P_{\eta}$ we obtain an $\mathcal{O}_{U(\eta)}^{\times}$-torsor $\left.\mathcal{L}_{\bar{m}} \subset\left(f^{*} \mathcal{M}_{X}\right)\right|_{U(\eta)}$. Then the extension $f^{b}$ of $f_{K}^{b}$ induces an isomorphism of $\mathcal{L}_{\bar{m}}$ with an $\mathcal{O}_{U(\eta)}^{\times}$-torsor in $\left.\mathcal{M}_{C}^{\prime}\right|_{U(\eta)}$. But $\left(\underline{C}, \mathcal{M}_{C}^{\prime}\right) \rightarrow\left(\operatorname{Spec} R, \mathcal{M}_{R}^{\prime}\right)$ is strict on $U(\eta)$, and hence this torsor is trivial and equal to the pull-back of an $\mathcal{O}_{R}^{\times}$-torsor in $\mathcal{M}_{R}^{\prime}$. Moreover, if $m \in \Gamma\left(U(\eta), \mathcal{L}_{\bar{m}}\right)$ maps to the pull-back of a section of $\mathcal{M}_{R}^{\prime}$ over Spec $K$, then there exists a unique $m^{\prime} \in \Gamma\left(\mathcal{M}_{R}^{\prime}\right)$ with $f^{b}(m)=\pi^{b}\left(m^{\prime}\right)$. The map $m \mapsto m^{\prime}$ defines the desired factorization $\mathcal{M}(\eta)_{\eta} \rightarrow \mathcal{M}_{R, 0}^{\prime} \rightarrow Q \times_{Q_{K}} \Gamma\left(\mathcal{M}_{K}\right)$.

4.3. Extension of the log morphism. Our log structure $\mathcal{M}_{R}$ comes with a morphism $\mathcal{M}_{R}^{0} \rightarrow \mathcal{M}_{R}$, which by the universal property of $\mathcal{M}_{R}^{0}$ extends the given structure of a log smooth curve on $\underline{C}_{K} \rightarrow \operatorname{Spec} K$ to $\underline{C} \rightarrow \operatorname{Spec} R$, in the category $(\log / S)$. Moreover, by uniqueness of $\mathcal{M}_{R, 0} \subset Q \times{ }_{Q_{K}} \Gamma\left(\mathcal{M}_{K}\right)$ this extension is uniquely isomorphic to the log structure on the domain $\underline{C}$ of any extension of $\left(C_{K} /\left(\operatorname{Spec} K, Q_{K}\right), \mathbf{x}_{K}, f_{K}\right)$ as a basic stable log map. Denote by $\varphi: \underline{f}^{*} \overline{\mathcal{M}}_{X} \rightarrow \overline{\mathcal{M}}_{C}$ the extension of $\overline{f_{K}^{b}}$ constructed in 4.1

Now if $U \subset \underline{C}$ is an open subset and $\bar{m} \in \Gamma\left(U, f^{*} \overline{\mathcal{M}}_{X}\right)$ we obtain two $\mathcal{O}_{U}^{\times}$-torsors in $\left.\underline{f}^{*} \mathcal{M}_{X}\right|_{U}$ and in $\left.\mathcal{M}_{C}\right|_{U}$, respectively. If $\mathcal{L}_{\bar{m}}$ and $\mathcal{L}_{\varphi(\bar{m})}$ are the corresponding 
line bundles the question is if the isomorphism

$$
\left.f_{K}^{b}\right|_{\mathcal{L}_{\bar{m}}}:\left.\left.\mathcal{L}_{\bar{m}}\right|_{\underline{C}_{K} \cap U} \longrightarrow \mathcal{L}_{\varphi(\bar{m})}\right|_{\underline{C}_{K} \cap U}
$$

extends to $U$, thus defining $\left.f^{b}\right|_{\mathcal{L}_{\bar{m}}}$ uniquely. Note that since $\underline{C}$ is Cohen-Macaulay any such extension is unique, and an extension exists iff it exists in codimension one, that is, at the generic points $\eta \in \underline{C}_{0}$. But if $m \in \mathcal{M}_{X, \underline{f}(\eta)}$ there exists $h \in \mathcal{O}_{\underline{C}, \eta}^{\times}$ with $m^{\prime}:=h \cdot m \in \mathcal{M}(\eta)_{\eta}$. Moreover, $\mathcal{M}_{C, \bar{\eta}}=\left(\underline{\pi}^{*} \mathcal{M}_{R, 0}\right)_{\bar{\eta}}$. Using the definition of $\mathcal{M}_{R, 0}$ we therefore see that

$$
f_{K}^{b}(m)=h^{-1} \cdot f_{K}^{b}\left(m^{\prime}\right)=h^{-1} \cdot \pi_{K}^{b}\left(\left(\psi_{\eta} \times \beta_{\eta}\right)\left(m^{\prime}\right)\right)
$$

extends over $\eta$ as $h^{-1} \cdot \pi^{b}\left(m^{\prime \prime}\right)$ with $m^{\prime \prime}:=\left(\psi_{\eta} \times \beta_{\eta}\right)\left(m^{\prime}\right) \in \mathcal{M}_{R, 0}$. This proves the unique existence of an extension $f$ of $f_{K}$ to $\underline{C}$, the last step in our proof of Theorem 4.1 .

\section{Log Gromov-Witten invariants}

We are now in position to define log Gromov-Witten invariants by constructing a virtual fundamental class on $\mathscr{M}(X)$. This is quite standard by now. We follow the method of Behrend and Fantechi $\mathrm{Be}$, BeFa with the improvement by Kresch $[\mathrm{Kr}$. The method has been adapted by Kim to the log setting Ki using Olsson's log cotangent complex Ol3. A low tech approach based on a global version of Artin's obstruction theory [Ar], close to the original approach of Li and Tian [LiTi] and avoiding cotangent complexes completely is also possible [Si1], [Si3], but this would be less economic.

Some remarks on logarithmic cotangent complexes are in order. An argument by $\mathrm{W}$. Bauer presented in $\mathrm{Ol} 3$, $\S 7$, shows that there is no theory of logarithmic cotangent complexes with the following four properties: (1) For strict morphisms one retrieves the ordinary cotangent complex. (2) For a log smooth morphism $X \rightarrow Y$ it is represented by $\Omega_{X / Y}^{1}$. (3) Functoriality. (4) Compositions of maps yields distinguished triangles. The point is that (1), (2) and (4) imply compatibility with base change of the ordinary cotangent complex $L_{\underline{Y}^{\prime} / \underline{Y}}^{\bullet}$ for a morphism of schemes $\underline{Y}^{\prime} \rightarrow \underline{Y}$ underlying an arbitrary log étale morphism $Y^{\prime} \rightarrow Y$. Since such morphisms need not be flat this is not true in general.

Olsson presents two ways out of this. The first method defines $L_{X / Y}^{\bullet}$ as the ordinary cotangent complex of the associated morphism of algebraic stacks $\underline{X} \rightarrow \log _{Y}$ (O13, Definition 3.2). This version of the logarithmic cotangent complex fulfills (1), (2) and (3), but (4) only holds under an additional assumption (Condition (T)). The second method, proposed by Gabber, works by enhancing the simplicial resolution approach to the cotangent complex by log structures $([\mathrm{Ol3}, \S 8)$. The resulting object $L_{X / Y}^{G, \bullet}$ fulfills (1), (3) and (4), but (2) only provided $f$ is integral. The two approaches agree in many respects (see Ol3], Theorem 8.32 and Corollary 8.34). In our case we need to represent the cotangent complex of $X \rightarrow S$ by a locally free sheaf but do not want to impose integrality of $X \rightarrow S$, and hence decided for the first version.

We should also point out that we need an extension of the theory developed in Ol3 for morphisms of log schemes to morphisms of log algebraic stacks with Deligne-Mumford domains. This should be a straightforward generalization, but since this statement is not available in the literature we will show in Remark 5.2 
how to reduce to the case of morphisms of log schemes by working on an étale cover. For the moment let us ignore this issue.

We now require $X \rightarrow S$ to be $\log$ smooth and quasi-projective. Let $\mathscr{U} \rightarrow \mathscr{M}$ be the universal curve over the $\log$ stack $\mathscr{M}$ of (not necessarily basic) pre-stable curves over $S$, as discussed in Appendix A. Let $\mathscr{M}(X)=\mathscr{M}(X / S)$ be the log stack of basic stable log maps over $S$,

$$
\pi: \mathscr{V}:=\mathscr{M}(X) \times \mathscr{M} \mathscr{U} \longrightarrow \mathscr{M}(X)
$$

the universal curve and

$$
f: \mathscr{V} \longrightarrow X
$$

the evaluation morphism, both considered as 1-morphisms of algebraic log stacks. The commutative diagram

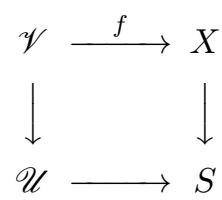

gives rise to the morphism of log cotangent complexes ([0l3], (1.1.2))

$$
L f^{*} L_{X / S}^{\bullet} \longrightarrow L_{\mathscr{V} / \mathscr{U}}^{\bullet}
$$

Now $\mathscr{V} \rightarrow \mathscr{U}$ is strict and $\underline{\pi}: \underline{\mathscr{V}} \rightarrow \mathscr{M}(X)$ is obtained from the flat morphism $\underline{\mathscr{U}} \rightarrow \underline{\mathscr{M}}$ by a base change. Hence, by $\overline{[\mathrm{Ol3}]}, 1.1$,(ii) and by the compatibility of the ordinary cotangent complex with flat base change ([I], II.2.2),

$$
L_{\mathscr{V} / \mathscr{U}}^{\bullet} \simeq L_{\underline{\mathscr{V}} / \underline{\mathscr{U}}}^{\bullet} L \pi^{*} L_{\underline{\mathscr{M}(X)} / \underline{\mathscr{M}}}^{\bullet}
$$

In contrast to previous usage we have to indicate now by underlining when we want to view $\mathscr{M}(X)$ or $\mathscr{M}$ as ordinary stacks rather than as log stacks. Tensoring with the relative dualizing sheaf $\omega_{\underline{\pi}}$ and using the fact that $L \pi^{!} L_{\underline{\mathscr{M}(X)} / \underline{\mathscr{M}}}^{\bullet} \simeq L \pi^{*} L_{\underline{\mathscr{M}(X))}}^{\bullet} \underline{\mathscr{M}}^{\otimes} \stackrel{L}{\otimes}$ $\omega_{\underline{\pi}}$, adjunction now defines a morphism

$$
\phi: R \pi_{*}\left(L f^{*} L_{X / S}^{\bullet} \stackrel{L}{\otimes} \omega_{\underline{\pi}}\right) \longrightarrow L_{\mathscr{M}(X) / \underline{\mathscr{M}}}^{\bullet} .
$$

By $\log$ smoothness $L_{X / S}^{\bullet}=\left[\Omega_{X / S}^{1}\right]([\mathrm{Ol3}, 1.1,(\mathrm{iii}))$ is represented by a locally free sheaf. Hence by duality, the left-hand side equals

$$
E^{\bullet}:=\left(R \pi_{*}\left[f^{*} \Theta_{X / S}\right]\right)^{\vee},
$$

which is of perfect amplitude contained in $[-1,0]$. We have thus constructed a morphism

$$
\phi: E^{\bullet} \longrightarrow L_{\underline{\mathscr{M}(X)}}^{\bullet} / \underline{\mathscr{M}} \cdot
$$

Proposition 5.1. The morphism (5.1) is a perfect obstruction theory relative to $\underline{\mathscr{M}}$ in the sense of $[\mathrm{BeFa}]$, Definition 4.4.

Proof. We are going to check the obstruction-theoretic criterion of $\mathrm{BeFa}$, Theorem 4.5,3, applied relative to $\underline{\mathscr{M}}$. To this end let $T \rightarrow \bar{T}$ be a square zero extension of schemes over $\mathscr{M}$ with ideal sheaf $\mathcal{J}$ and $g: T \rightarrow \mathscr{M}(X)$. We consider $T$ and $\bar{T}$ as endowed with the log structures making the morphisms to $\mathscr{M}$ strict. Denote by $\mathscr{V}_{T}=T \times \mathscr{M}(X) \mathscr{V}=T \times \mathscr{M} \mathscr{U}, \mathscr{V}_{\bar{T}}=\bar{T} \times \mathscr{M} \mathscr{U}$, and by $p: \mathscr{V}_{T} \rightarrow T, \tilde{g}: \mathscr{V}_{T} \rightarrow \mathscr{V}$ 
the projections. Let $f_{T}:=f \circ \tilde{g}: \mathscr{V}_{T} \rightarrow X$ be the induced log morphism. We are thus led to the following commutative diagram:

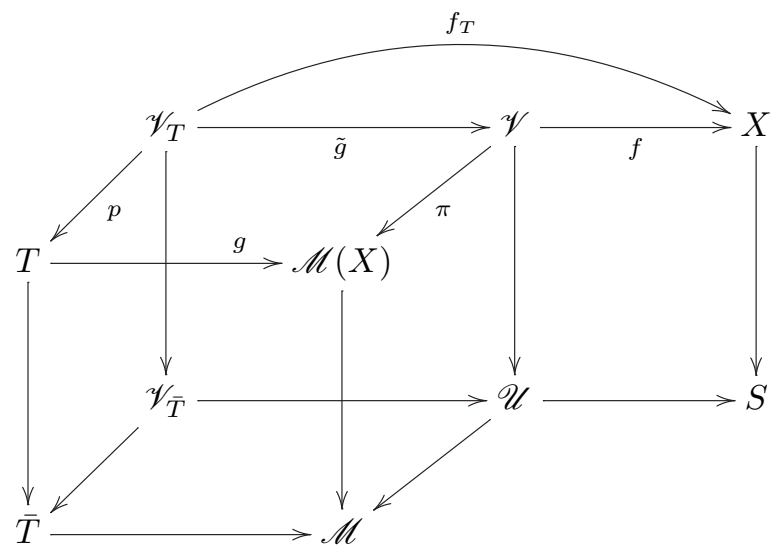

Note that all but the front and back faces of the cube are cartesian. Because $\mathscr{M}(X) \rightarrow \mathscr{M}$ is strict and representable standard obstruction theory applies to the extension problem of $g$ to $\bar{T}$ (II], Ch.III, and O14 for the case of representable morphisms of stacks). Thus the obstructions class $\omega(g) \in \operatorname{Ext}_{\mathcal{O}_{T}}^{1}\left(L g^{*} L_{\mathscr{M}(X) / \mathscr{M}}^{\bullet}, \mathcal{J}\right)$ to the existence of an extension is given by the composition

$$
L g^{*} L_{\mathscr{\mathscr { M }}(X) / \underline{\mathscr{M}}} \longrightarrow L_{T / \bar{T}}^{\bullet} \longrightarrow \tau_{\geq-1} L_{T / \bar{T}}^{\bullet}=\mathcal{J}[1],
$$

defined by functoriality of the cotangent complex. Now BeFa], Theorem $5.3,3$, says that $\phi$ defines an obstruction theory in the sense of Behrend and Fantechi if the following hold for any extension problem. (1) An extension exists if and only if $\phi^{*} \omega(g)=0$, and (2) in this case the set of isomorphism classes of extensions form a torsor under $\operatorname{Hom}_{\mathcal{O}_{T}}\left(L g^{*} E^{\bullet}, \mathcal{J}\right)$. Here $\phi^{*} \omega(g) \in \operatorname{Ext}_{\mathcal{O}_{T}}^{1}\left(L g^{*} E^{\bullet}, \mathcal{J}\right)$ is the image of $\omega(g)$ under pull-back by $\phi$.

On the other hand, by the definition of $\mathscr{M}(X)$, such an extension exists if and only if $f_{T}: \mathscr{V}_{T} \rightarrow X$ extends as a $\log$ morphism to $\mathscr{V}_{\bar{T}}$. Ol3, Theorem 8.45, provides the obstruction theory for this situation. From this point of view there is an obstruction class $o \in \operatorname{Ext}_{\mathcal{O}_{\mathscr{V}_{T}}}^{1}\left(L f_{T}^{*} L_{X / S}^{\bullet}, p^{*} \mathcal{J}\right)$, and the isomorphism classes of extensions form a torsor under $\operatorname{Hom}_{\mathcal{O}_{\mathscr{V}_{T}}}\left(L f_{T}^{*} L_{X / S}^{\bullet}, p^{*} \mathcal{J}\right)$. The obstruction class $o$ is defined by the morphism 13

$$
L f_{T}^{\bullet} L_{X / S}^{\bullet} \longrightarrow L_{\mathscr{V}_{T} / S}^{\bullet} \longrightarrow L_{\mathscr{V}_{T} / \mathscr{V}_{\bar{T}}}^{\bullet} \rightarrow \tau_{\geq-1} L_{\mathscr{V}_{T} / \mathscr{V}_{\bar{T}}}^{\bullet}=\mathcal{J}[1]
$$

To compare the two obstruction situations note that repeated application of adjunction yields the following sequence of identifications, for any $k \in \mathbb{N}$ :

$$
\begin{aligned}
\operatorname{Ext}_{\mathcal{O}_{T}}^{k}\left(L g^{*} E^{\bullet}, \mathcal{J}\right) & =\operatorname{Ext}_{\mathcal{O}_{T}}^{k}\left(L g^{*} R \pi_{*}\left(L f^{*} L_{X / S}^{\bullet} \stackrel{L}{\otimes} \omega_{\pi}\right), \mathcal{J}\right) \\
& =\operatorname{Ext}_{\mathcal{O}_{\mathscr{V}}}^{k}\left(L f^{*} L_{X / S}^{\bullet} \stackrel{L}{\otimes} \omega_{\pi}, L \pi^{!}\left(R g_{*} \mathcal{J}\right)\right) \\
& =\operatorname{Ext}_{\mathcal{O}_{\mathscr{V}}}^{k}\left(L f^{*} L_{X / S}^{\bullet}, R \tilde{g}_{*}\left(p^{*} \mathcal{J}\right)\right)=\operatorname{Ext}_{\mathcal{O}_{\mathscr{V}_{T}}}^{k}\left(L f_{T}^{*} L_{X / S}^{\bullet}, p^{*} \mathcal{J}\right) .
\end{aligned}
$$

\footnotetext{
${ }^{13}$ The proof of [Ol3], Theorem 8.45, proceeds with Gabber's version of the cotangent complex, but in view of [Ol3], Theorem 8.32, this has no influence on the obstruction class.
} 
For the third equality we used $L \pi^{!}=L \pi^{*} \otimes \omega_{\pi}$ and $L \pi^{*} \circ R g_{*}=R \tilde{g}_{*} \circ L p^{*}$, the latter by flatness of $\pi$. Tracing through this sequence of isomorphisms for $k=1$ now indeed maps $\phi^{*} \omega(g)$ to the obstruction morphism $o$ in (5.3). This proves the obstruction part of the criterion in $[\mathrm{BeFa}$, Theorem 5.3,3. The torsor part follows readily from (5.4) with $k=0$.

Finally, by $[\mathrm{BeFa}, \S 5$ and $[\mathrm{Kr}$, Theorem 6.2 .1 , we now have a virtual fundamental class $\llbracket \mathscr{M}(X / S) \rrbracket$, as a well-defined rational Chow class on $\mathscr{M}(X / S)$. Moreover, if $\beta$ is a class of stable $\log$ maps to $X$ fulfilling the maximality condition of Definition 3.1 (ii), then $\mathscr{M}(X / S, \beta) \subset \mathscr{M}(X / S)$ is an open substack and hence also carries a virtual fundamental class.

Remark 5.2. To reduce to an étale cover let us recall the construction of the virtual fundamental class in $\mathrm{BeFa}$. Behrend and Fantechi first construct a canonical cone stack $\mathfrak{C}_{Z / S}$ for any morphism of Artin stacks $Z \rightarrow S$ that is relatively DeligneMumford, the intrinsic normal cone. It is a closed substack of the intrinsic normal sheaf $\mathfrak{N}_{Z / S}$, a Picard stack over $Z$ (that is, it has an additive structure relative to $Z)$. Both stacks are functorial for étale morphisms by $[\mathrm{BeFa}$, Proposition 3.14. A perfect obstruction theory $E^{\bullet} \rightarrow L_{Z / S}^{\bullet}$ then provides a closed embedding $\mathfrak{C}_{Z / S} \rightarrow$ $h^{1} / h^{0}\left(E^{\vee}\right)$ into a vector bundle stack, where we use the notation of [BeFa]. Again, this embedding is compatible with étale morphisms, so can be constructed on an étale cover. One then obtains a cone stack of pure dimension 0 inside the vector bundle stack $h^{1} / h^{0}\left(E^{\vee}\right)$ over $\mathscr{M}(X)$. Intersecting with the zero section as defined in $[\mathrm{Kr}]$ then defines the virtual fundamental class.

The theory of log cotangent complexes only enters in the construction of the perfect obstruction theory. Since the obstruction theory is compatible with étale morphisms we can go over to an étale cover and work with diagrams of log schemes rather than stacks.

\section{The RELATIONSHIP WITH EXPANDED DEGENERATIONS}

Let $\underline{X}$ be a non-singular variety and $D \subset \underline{X}$ a smooth divisor, and define $X$ as the $\log$ scheme with the divisorial $\log$ structure $\mathcal{M}_{(X, D)}$. This is the case of relative Gromov-Witten invariants considered by [LiRu, [IoPa], Ga], Li1]. It is insightful to compare the moduli space of stable log maps in this context with the moduli space constructed by Jun Li.

Conjecturally, the Gromov-Witten invariants defined using these moduli spaces will coincide, though we will make no attempt to prove this here14. On the other hand, the moduli spaces themselves are demonstrably not isomorphic.

We will sketch here the relationship between these moduli spaces, assuming familiarity with Li's notion of stable relative maps. Throughout this section "fs" stands for "fine, saturated".

Let $\mathcal{M}_{X}$ be the divisorial $\log$ structure on $\underline{X}$ defined by $D, \beta$ a class of stable $\log$ maps, $X=\left(\underline{X}, \mathcal{M}_{X}\right)$, and let $\mathscr{M}(X, \beta)$ be as usual the stack of basic stable log maps of class $\beta$. Let $\mathbf{M}(\underline{X} / D, \beta)$ be Jun Li's moduli space of stable relative maps. In fact, Jun Li constructed a log structure on this stack, but it is not saturated, and as a consequence, we cannot get a morphism $\mathbf{M}(\underline{X} / D, \beta) \rightarrow \mathscr{M}(X, \beta)$. Rather, one must pass to the saturation $\mathbf{M}(\underline{X} / D, \beta)^{\text {sat }}$, constructed using $\mathrm{Og}$, II, Prop. 2.4.5. We will then obtain a morphism of fs $\log$ stacks $\Psi: \mathbf{M}(\underline{X} / D, \beta)^{\text {sat }} \rightarrow \mathscr{M}(X, \beta)$.

\footnotetext{
${ }^{14}$ Note added in final revision: This statement has now been proved AMW].
} 
Recall a family of relative stable maps over a base scheme $\underline{W}$ is given by the following data. For each $n \geq 0$, there is a pair $(\underline{X}[n], D[n])$ constructed from $(\underline{X}, D)$ where $\underline{X}[n]$ is defined over $\mathbb{A}^{n}$ and $D[n] \subset \underline{X}[n]$ is a divisor. Then a family of relative stable maps is a diagram

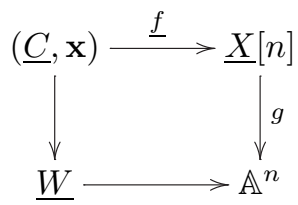

which is a family of ordinary stable maps in $\underline{X}[n]$ satisfying certain conditions (predeformability and finiteness of a certain notion of automorphism group), and has specified tangencies with $D[n]$. In $\S 1.3$ of $\left[\mathrm{Li2}\right.$, Li observes that $\underline{X}[n]$ and $\mathbb{A}^{n}$ carry canonical $\log$ structures. The $\log$ structure on $\mathbb{A}^{n}$ is the divisorial log structure associated to the divisor $B$ given by $t_{1} \cdots t_{n}=0$, and the one on $\underline{X}[n]$ is induced by $g^{-1}(B) \cup D[n]$. Furthermore, there is a natural projection $\underline{\Theta}: \underline{X}[n] \rightarrow \underline{X}$ such that $\underline{\Theta}^{-1}(D) \subset g^{-1}(B) \cup D[n]$, hence giving rise to a $\log$ morphism $\Theta: X[n] \rightarrow X$. Li then constructs $\log$ structures on $\underline{C}$ and $\underline{W}$ making (6.1) into a commutative diagram of $\log$ schemes. Now $C \rightarrow W$ is a $\log$ smooth curve with marked points along $\underline{f}^{-1}(D[n])$.

To pass between relative stable maps and stable log maps, we need the following proposition:

Proposition 6.1. There is a commutative diagram of fine log schemes

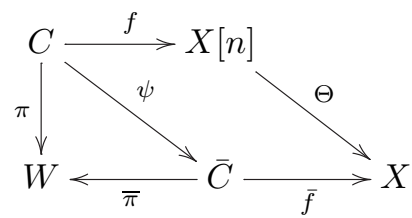

so that $(\bar{C} / W, \mathbf{x}, \bar{f})$ satisfies all conditions of being a stable log map except that the log structures on $\bar{C}$ and $W$ need not be saturated.

Proof. It is standard that such a diagram exists at the level of schemes, with $\bar{f}$ a stabilization of $\underline{\Theta} \circ \underline{f}$ ([BeMa], Theorem 3.6). The map $\underline{\psi}$ contracts in every fibre of $\underline{\pi}$ every $\mathbb{P}^{1}$ component with only two special points on which $\underline{\Theta} \circ f$ is constant. We need to understand these morphisms at the log level.

Step 1. Review of $g: X[n] \rightarrow X$. The pair $(\underline{X}[n], D[n])$ is constructed inductively: $(\underline{X}[0], D[0])$ is the pair $(\underline{X}, D)$. Then the pair $(\underline{X}[n], D[n])$ is obtained by blowing up $\underline{X}[n-1] \times \mathbb{A}^{1}$ along $D[n-1] \times\{0\}$, and $D[n]$ is the proper transform of $D[n-1] \times \mathbb{A}^{1}$. If $D$ is given by the vanishing of a regular function $w_{0}=0$, (which can always be accomplished locally on $\underline{X}$ ), then in fact $\underline{X}[n]$ can be described as the subscheme of $\underline{X} \times\left(\mathbb{P}^{1}\right)^{n} \times \mathbb{A}^{n}$ given by the equations

$$
w_{0} z_{1}=w_{1} t_{1}, \quad w_{1} z_{2}=w_{2} z_{1} t_{2}, \quad \ldots, \quad w_{n-1} z_{n}=z_{n-1} w_{n} t_{n},
$$

where $z_{i}, w_{i}$ are homogeneous coordinates on the $i$-th copy of $\mathbb{P}^{1}$ and $t_{1}, \ldots, t_{n}$ are coordinates on $\mathbb{A}^{n}$. This is covered by affine open subsets $\underline{X}_{i}, 1 \leq i \leq n+1$, where $\underline{X}_{i}$ is given by $z_{1}=\cdots=z_{i-1}=1=w_{i}=\cdots=w_{n}$. Thus $w_{i-1} z_{i}=t_{i}$ on $\underline{X}_{i}$ for 
$i \leq n$. The $\log$ structure of $X[n]$ restricted to $\underline{X}_{i}$ for $i \leq n$ has a chart

$$
\mathbb{N}^{n} \oplus_{\mathbb{N}} \mathbb{N}^{2} \rightarrow \mathcal{O}_{X_{i}}
$$

where the map $\mathbb{N} \rightarrow \mathbb{N}^{n}$ is $1 \mapsto e_{i}$, the $i$-th generator of $\mathbb{N}^{n}$, and the chart is

$$
\left(\sum_{i=1}^{n} a_{i} e_{i},(a, b)\right) \longmapsto\left(\prod_{i=1}^{n} t_{i}^{a_{i}}\right) w_{i-1}^{a} z_{i}^{b} .
$$

The chart on $X_{n+1}$ is just $\mathbb{N}^{n} \oplus \mathbb{N} \rightarrow \mathcal{O}_{X_{n+1}}$ given by $\left(\sum a_{i} e_{i}, a\right) \mapsto w_{n}^{a} \prod t_{i}^{a_{i}}$.

Recall that $X[n]$ fibres over $\mathbb{A}^{n}$ with coordinates $t_{1}, \ldots, t_{n}$, and that the critical locus of this map consists of $n$ distinct subvarieties $D_{1}, \ldots, D_{n}$, with $D_{i}$ sitting over $t_{i}=0$. In the above description, $D_{i} \subset X_{i}$ is given by $w_{i-1}=z_{i}=0$. Also, $D[n] \subset X_{n+1}$ is given by $w_{n}=0$.

The map $\Theta: X[n] \rightarrow X$ is the projection to $X$. We describe this as a log morphism as follows. Let $s_{w_{0}}$ be the section of $\mathcal{M}_{X}$ corresponding to the function $w_{0}$ vanishing only along $D$. In general, if we have a chart $P \rightarrow \mathcal{O}_{Y}$ for a $\log$ structure, for $m \in P$ we denote by $s_{m}$ the corresponding section of the associated $\log$ structure, so that any section of the associated log structure is of the form $h \cdot s_{m}$ for some $m \in P, h \in \mathcal{O}_{Y}^{\times}$. Then $\Theta: X_{i} \rightarrow X$ is given by (for $i \leq n$ )

$$
s_{w_{0}} \longmapsto s_{\left(e_{1}+\cdots+e_{i-1},(1,0)\right)}
$$

since on $\underline{X}_{i}, w_{0}=w_{i-1} t_{1} \cdots t_{i-1}$, while $\Theta: X_{n+1} \rightarrow X$ is given by

$$
s_{w_{0}} \longmapsto s_{\left(e_{1}+\cdots+e_{n}, 1\right)} .
$$

Step 2. Review of $C \rightarrow W$. Fix a geometric point $\bar{w} \in|W|$. Let $\mathbf{D}$ be the set of distinguished double points of $\underline{C}_{\bar{w}}$, that is, double points mapping to $\bigcup_{\ell=1}^{n} D_{\ell}$, with $\mathbf{D}_{\ell} \subset \mathbf{D}$ the subset mapping to $D_{\ell}$. Let $\mathbf{U}$ be the set of undistinguished double points. For each $q \in \mathbf{D}_{\ell}$, let $\mu_{q}$ denote the order of tangency of either branch of $C_{\bar{w}}$ at $q$ with $D_{\ell}$. Let $N_{\ell}$ be the free monoid $\mathbb{N} \rho_{\ell}$ generated by $\rho_{\ell}$ if $\mathbf{D}_{\ell}=\emptyset$; otherwise $N_{\ell}$ is the monoid generated by $\left\{\rho_{q} \mid q \in \mathbf{D}_{\ell}\right\}$ modulo the relations $\mu_{q} \rho_{q}=\mu_{q^{\prime}} \rho_{q^{\prime}}$ for each $q, q^{\prime} \in \mathbf{D}_{\ell}$. Then 15

$$
\overline{\mathcal{M}}_{W, \bar{w}}=\bigoplus_{\ell=1}^{n} N_{\ell} \oplus \bigoplus_{q \in \mathbf{U}} \mathbb{N} \rho_{q} .
$$

Note the monoids $N_{\ell}$ need not be saturated; as a consequence, the log structures on $W, C$ and $\bar{C}$ we construct below need not be saturated.

For every point $q \in \mathbf{D}_{\ell}$, one can find an étale open neighbourhood $U_{q}$ of $q \in \underline{C}$ such that $\underline{f}$ maps $U_{q}$ into $\underline{X}_{\ell}$. Furthermore, $U_{q}$ is of the form $\operatorname{Spec} A\left[x_{q}, y_{q}\right] /\left(x_{q} y_{q}-\right.$ $t_{q}$ ) where étale locally $W=\operatorname{Spec} A$, and $t_{q} \in A$. As observed in [Li2], Simplification 1.7, we can choose $U_{q}$ and coordinates $x_{q}, y_{q}$ so that

Then $\underline{f}^{*}\left(t_{\ell}\right)=t_{q}^{\mu_{q}}$.

$$
\underline{f}^{*}\left(w_{\ell-1}\right)=x_{q}^{\mu_{q}}, \quad \underline{f}^{*}\left(z_{\ell}\right)=y_{q}^{\mu_{q}} .
$$

Similarly, for each $q \in \mathbf{U}$, we can describe a neighbourhood $U_{q}$ of $q$ in $\underline{C}$ as

$$
U_{q} \cong \operatorname{Spec} A\left[x_{q}, y_{q}\right] /\left(x_{q} y_{q}-t_{q}\right)
$$

for some $t_{q} \in A$.

\footnotetext{
${ }^{15} \mathrm{Jun} \mathrm{Li}$ does not include the contributions from the undistinguished nodes; these must be included in order for $C \rightarrow W$ to be $\log$ smooth. However, these contributions will play no further role in the discussion.
} 
Given these choices, Li puts log structures on $C$ and $W$ as follows. There is a chart for the log structure of $W$,

$$
\sigma: \overline{\mathcal{M}}_{W, \bar{w}} \longrightarrow A
$$

given by

$$
\rho_{q} \longmapsto t_{q}, \quad \rho_{\ell} \longmapsto \underline{h}^{*} t_{\ell}
$$

where $\underline{h}: \underline{W} \rightarrow \mathbb{A}^{n}$. A chart for the $\log$ structure on $C$ for the neighbourhood $U_{q}$ is

$$
\begin{aligned}
\psi_{q}: \overline{\mathcal{M}}_{W, \bar{w}} \oplus_{\mathbb{N}} \mathbb{N}^{2} \longrightarrow \mathcal{O}_{U_{q}} \\
(\alpha,(a, b)) \longmapsto \underline{\pi}^{*}(\sigma(\alpha)) x_{q}^{a} y_{q}^{b}
\end{aligned}
$$

Here the map $\mathbb{N} \rightarrow \overline{\mathcal{M}}_{W, \bar{w}}$ is $1 \mapsto \rho_{q}$.

If $p$ is any marked point of $\underline{C}$ with $\underline{f}(p) \in D[n]$, then in a suitable neighbourhood $U_{p}=\operatorname{Spec} A\left[x_{p}\right]$ of $p$, with chart $\overline{\mathcal{M}}_{W, \bar{w}} \oplus \mathbb{N} \rightarrow A\left[x_{p}\right]$ given as usual as $(\alpha, a) \mapsto$ $\pi^{*}(\sigma(\alpha)) x_{p}^{a}$. We have $\underline{f}^{*}\left(w_{n}\right)=x_{p}^{\mu_{p}} \cdot h_{p}$ for a unit $h_{p}$ and some $\mu_{p} \in \mathbb{N}$, which will be assumed to be 1 via a suitable choice of $U_{p}$ and $x_{p}$.

One checks these induced log structures do not depend on any choices and glue uniquely, yielding a well-defined $C \rightarrow W$.

Step 3. The $\log$ morphism $f: C \rightarrow X[n]$. For $q \in \mathbf{D}_{i}, f:\left(U_{q},\left.\mathcal{M}_{C}\right|_{U_{q}}\right) \rightarrow X_{i}$ is given by

$$
s_{\left(\sum a_{\ell} e_{\ell},(a, b)\right)} \longmapsto s_{\left(\sum a_{\ell} \rho_{\ell}^{\prime},\left(\mu_{q} a, \mu_{q} b\right)\right)}
$$

where $\rho_{\ell}^{\prime}=\rho_{\ell}$ if $\mathbf{D}_{\ell}$ is empty and is equal to $\mu_{q} \rho_{q}$ for any $q \in \mathbf{D}_{\ell}$ otherwise. If $p$ is a marked point of $\underline{C}$ with $\underline{f}(p) \in D[n]$, then $f$ is given on $\left(U_{p},\left.\mathcal{M}_{C}\right|_{U_{p}}\right) \rightarrow X_{n+1}$ by

$$
s_{\left(\sum a_{\ell} e_{\ell}, a\right)} \longmapsto s_{\left(\sum a_{\ell} \rho_{\ell}^{\prime}, \mu_{p} a\right)} .
$$

This is sufficient to completely specify $f$.

Step 4. The map $C \rightarrow \bar{C}$. The $\log$ structure on $\bar{C}$ can be described as follows. First, if $\underline{\bar{C}}^{O} \subset \underline{\bar{C}}$ is the largest open set for which $\underline{\psi}^{-1}\left(\underline{\bar{C}}^{o}\right) \rightarrow \underline{\bar{C}}^{o}$ is an isomorphism, $\bar{C}^{o}$ has the same log structure as $\psi^{-1}\left(\bar{C}^{o}\right)$.

Next, let $\bar{y} \in|\underline{\bar{C}}|$ be a geometric point not in $\underline{\bar{C}}^{o}$, with $\bar{w}=\underline{\bar{\pi}}(\bar{y})$. There are two cases: $\bar{y}$ either is not, or is, a double point of $\underline{\bar{C}}_{\bar{w}}$. In both cases, $\psi^{-1}(\bar{y})$ is a chain $\underline{C}_{1} \cup \cdots \cup \underline{C}_{m}$ of rational curves, with $q_{i}=\underline{C}_{i} \cap \underline{C}_{i+1}$ a double point, and $\underline{C}_{\bar{w}}$ has another component $\underline{C}_{0}$ with $\underline{C}_{0} \cap \underline{C}_{1}=q_{0}$ also a double point. However, if $\bar{y}$ is not a double point, then there is a point $p_{m} \in \underline{C}_{m}$ which is a log marked point, while if $\bar{y}$ is a double point, $\underline{C}_{\bar{w}}$ has a component $\underline{C}_{m+1}$ and a double point $q_{m}=\underline{C}_{m} \cap \underline{C}_{m+1}$.

If $\bar{y}$ is not a double point, then there is an étale open neighbourhood of $\bar{y}$ of the form $U:=\operatorname{Spec} A[x]$, where $x=0$ is the image of the section $\underline{\psi} \circ x_{i}$, with $x_{i}$ the marked point of $\underline{C}$ corresponding to $p_{m}$.

If $\bar{y}$ is a double point, then one can show that there is an étale open neighbourhood of $\bar{y}$ of the form $U:=\operatorname{Spec} A[x, y] /\left(x y-\prod_{i=0}^{m} t_{q_{i}}\right)$. 
In both cases, one can describe $U \times_{\bar{C}} \underline{C}$. In the case that $\bar{y}$ is not a double point, then this scheme is given by the equations

$$
x u_{1}=v_{1} t_{q_{0}}, \quad v_{1} u_{2}=u_{1} v_{2} t_{q_{1}}, \quad \ldots, \quad v_{m-1} u_{m}=u_{m-1} v_{m} t_{q_{m-1}}
$$

in $\mathbb{A}^{1} \times\left(\mathbb{P}^{1}\right)^{m} \times \operatorname{Spec} A$, with homogeneous coordinates on the $i$-th $\mathbb{P}^{1}$ being $u_{i}, v_{i}$. If $\bar{y}$ is a double point, then this scheme is given by the equations

$$
x u_{1}=v_{1} t_{q_{0}}, \quad v_{1} u_{2}=u_{1} v_{2} t_{q_{1}}, \quad \ldots, \quad v_{m-1} u_{m}=u_{m-1} v_{n} t_{q_{m-1}}, \quad y v_{m}=u_{m} t_{q_{m}}
$$

in $\mathbb{A}^{2} \times\left(\mathbb{P}^{1}\right)^{m} \times \operatorname{Spec} A$.

We can cover $U \times_{\bar{C}} \underline{C}$ with Zariski open subsets $U_{i}$ for $0 \leq i \leq m$, where $U_{i}$ is the set where $u_{1}=\cdots=u_{i}=1=v_{i+1}=\cdots=v_{m}$. Note $U_{i}$ is an open neighbourhood of $q_{i}, U_{i} \cong \operatorname{Spec} A\left[v_{i}, u_{i+1}\right] /\left(v_{i} u_{i+1}-t_{q_{i}}\right)$ if $i \leq m$, and $U_{m} \cong \operatorname{Spec} A\left[v_{m}\right]$ if $\bar{y}$ is not a double point.

We have to be slightly careful with the coordinates $x$ and $y$; these cannot be chosen arbitrarily. To do so we have to relate these to the map $f$. At this point, we shall make a simplifying assumption that $\bar{w} \in W$ maps to $0 \in \overline{\mathbb{A}^{n}}$. This can always be achieved locally on $W$ by decreasing $n$, and the general case can be dealt with by the reader with some extra bookkeeping.

Possibly after reversing the order of $\underline{C}_{0}, \ldots, \underline{C}_{m+1}$ in the case that $\bar{y}$ is a double point, we can assume that there is some positive integer $\ell_{0}$ such that $\underline{f}\left(q_{i}\right) \in D_{\ell_{0}+i}$ for $i \leq m$, and $\underline{f}\left(p_{m}\right) \in \underline{X}_{n+1} \cap D[n]$ if $\bar{y}$ is not a double point. In the latter case, $\ell_{0}=n-m+1$. The map $\underline{f}: U_{i} \rightarrow \underline{X}[n]$ factors through $\underline{X}_{\ell_{0}+i}$, and necessarily yields

$$
\begin{aligned}
& i=0: \quad \underline{f}^{*}\left(w_{\ell_{0}-1}\right)=h_{0,1} x^{\mu}, \quad \underline{f}^{*}\left(z_{\ell_{0}}\right)=h_{0,2} u_{1}^{\mu}, \\
& 0<i<m: \quad \underline{f}^{*}\left(w_{\ell_{0}+i-1}\right)=h_{i, 1} v_{i}^{\mu}, \quad \underline{f}^{*}\left(z_{\ell_{0}+i}\right)=h_{i, 2} u_{i+1}^{\mu}, \\
& i=m: \quad \underline{f}^{*}\left(w_{\ell_{0}+m-1}\right)=h_{m, 1} v_{m}^{\mu}, \quad \underline{f}^{*}\left(z_{\ell_{0}+m}\right)=h_{m, 2} y^{\mu},
\end{aligned}
$$

the last line if $\bar{y}$ is a double point. Here $\mu=\mu_{q_{i}}$ for any $i$. If $\bar{y}$ is not a double point, then $U_{m} \rightarrow \underline{X}[n]$ factors through $X_{n+1}$, with

$$
f^{*}\left(w_{n}\right)=h_{m, 1} v_{m}^{\mu}
$$

Now in fact we can assume $h_{0,2}=h_{m, 1}=1, h_{i, k}=1$ for $0<i<m, k=1,2$. This is because $f$ on each component $\underline{C}_{i}, 1 \leq i \leq m$, is just a $\mu$-fold cover of $\mathbb{P}^{1}$ totally ramified at 0 and $\infty$, so that the above listed $h_{i, k}$ 's are constant, and then after applying a suitable change of coordinates on $\underline{C}$, one can assume these constants are 1 . Further, by making a change of coordinates for $x$ (and $y$ ), one can assume $h_{0,1}=1$ (and $h_{m, 2}=1$ in the double point case).

In particular, one can then assume that the open sets $U_{q_{0}}, \ldots, U_{q_{m-1}}, U_{p_{m}}$ if $\bar{y}$ is not a double point $\left(U_{q_{0}}, \ldots, U_{q_{m}}\right.$ if $\bar{y}$ is a double point) are taken to be the open sets $U_{0}, \ldots, U_{m}$. Thus we know what the chart for the $\log$ structure on $U_{i}$ is, by Step 2. This is given on $U_{i}$ for $i \leq m-1$ (and for $i=m$ in the case of a double point) by charts $\overline{\mathcal{M}}_{W, \bar{w}} \oplus_{\mathbb{N}} \mathbb{N}^{2} \rightarrow \mathcal{O}_{U_{i}}$ given by

$$
(\alpha,(a, b)) \longmapsto \underline{\pi}^{*}(\sigma(\alpha)) v_{i}^{a} u_{i+1}^{b},
$$

and on $U_{m}$ for $\bar{y}$ not a double point by a chart $\overline{\mathcal{M}}_{W, \bar{w}} \oplus \mathbb{N} \rightarrow \mathcal{O}_{U_{m}}$ given by

$$
(\alpha, a) \longmapsto \underline{\pi}^{*}(\sigma(\alpha)) v_{m}^{a}
$$


Using this particular choice of the coordinate $x$ (and $y$ if $\bar{y}$ is a double point), we can put a log structure on $U$ as follows. If $\bar{y}$ is not a double point, we have a chart $\overline{\mathcal{M}}_{W, \bar{w}} \oplus \mathbb{N} \rightarrow \mathcal{O}_{U}$ given by

$$
(\alpha, a) \longmapsto \underline{\bar{\pi}}^{*}(\sigma(\alpha)) \cdot x^{a} .
$$

If $\bar{y}$ is a double point, we have a chart

$$
\begin{aligned}
\overline{\mathcal{M}}_{W, \bar{w}} \oplus_{\mathbb{N}} \mathbb{N}^{2} & \longrightarrow \mathcal{O}_{U} \\
(\alpha,(a, b)) & \longmapsto \underline{\pi}^{*}(\sigma(\alpha)) x^{a} y^{b},
\end{aligned}
$$

with the map $\mathbb{N} \rightarrow \overline{\mathcal{M}}_{W, \bar{w}}$ given by $1 \mapsto \sum_{i=1}^{m} \rho_{q_{i}}$. It is straightforward to check that this $\log$ structure is independent of choices and this description gives compatible $\log$ structures for different choices of the point $\bar{y}$. Hence one obtains a log structure on $\underline{\bar{C}}$ clearly making $\bar{\pi} \log$ smooth.

We can specify the $\log$ morphism $\psi: U_{i} \rightarrow U$ as follows. If $\bar{y}$ is not a double point, this map is defined as

$$
s_{(\alpha, a)} \longmapsto \begin{cases}s_{\left(\alpha+a \sum_{j<i} \rho_{q_{j}},(a, 0)\right)} & i<m, \\ s_{\left(\alpha+a \sum_{j<m} \rho_{q_{j}}, a\right)} & i=m .\end{cases}
$$

On the other hand, if $\bar{y}$ is a double point, then for every $i$, we take the map

$$
s_{(\alpha,(a, b))} \longmapsto s_{\left(\alpha+a \sum_{j<i} \rho_{q_{j}}+b \sum_{j>i} \rho_{q_{j}},(a, b)\right)} .
$$

Using the explicit description for the charts on the sets $U_{i}$, one checks that the morphisms $U_{i} \rightarrow U$ agree on overlaps and hence give a morphism $U \times{ }_{\bar{C}} C \rightarrow$ $U$. Furthermore, these morphisms are compatible for different choices of open neighbourhoods $U$ of different points $\bar{y}$, giving the $\log$ morphism $\psi: C \rightarrow \bar{C}$.

Step 5. The map $\bar{f}: \bar{C} \rightarrow X$. We now define the $\log$ map $\bar{f}: U \rightarrow X$ by

$$
s_{w_{0}} \longmapsto \begin{cases}s_{\left(\sum_{\ell=1}^{\ell_{0}-1} \rho_{\ell}^{\prime}, \mu\right)} \in \mathcal{M}_{U} & \text { if } \bar{y} \text { is not a double point, } \\ s_{\left(\sum_{\ell=1}^{\ell_{0}-1} \rho_{\ell}^{\prime},(\mu, 0)\right)} \in \mathcal{M}_{U} & \text { if } \bar{y} \text { is a double point, }\end{cases}
$$

where $\rho_{\ell}^{\prime}$ is defined in Step 3. One checks from the description of all the maps above that this yields the desired commutative diagram, at least where these maps are now defined. Further, one checks that all maps are independent of choices and glue, hence giving the desired global commutative diagram.

By $\mathrm{Og}$, II 2.4.5, for any fine log scheme $W$ there is a finite surjective morphism $W^{\text {sat }} \rightarrow W$ from an fs $\log$ scheme $W^{\text {sat }}$, such that every morphism $W^{\prime} \rightarrow W$ with $W^{\prime}$ fine and saturated has a unique factorization through $W^{\text {sat }} \rightarrow W$. Thus we can define an fs log stack $\mathbf{M}(\underline{X} / D, \beta)^{\text {sat }}$ by defining an object over a scheme $\underline{W}$ to be a choice of relative stable map $f: C / W \rightarrow X[n]$ (with log structures as defined by Jun Li), yielding a log scheme $W^{\text {sat }}$, and a choice of section $\underline{W} \rightarrow \underline{W}^{\text {sat }}$ of $\underline{W}^{\text {sat }} \rightarrow \underline{W}$. Denote by $W^{\prime}$ the pull-back fs log structure on $\underline{W}$ from $W^{\text {sat }}$ under this morphism. Then from this data, using Proposition 6.1, we obtain a stable log map $\bar{C} \times_{W} W^{\prime} \rightarrow X$ over $W^{\prime}$. Thus we obtain:

Corollary 6.2. There is a morphism of stacks $\Psi: \mathbf{M}(\underline{X} / D, \beta)^{\mathrm{sat}} \rightarrow \mathscr{M}(X, \beta)$.

We shall see in the next section that this morphism is not in general expected to be an isomorphism. 


\section{EXAMPLES}

Example 7.1. Let $\underline{X}$ be a non-singular variety with a smooth divisor $D \subset \underline{X}$ as in the previous section, yielding the log scheme $X$ with the divisorial log structure. Consider first the case where $\underline{C}$ is a smooth curve and $\underline{f}: \underline{C} \rightarrow \underline{X}$ is an ordinary stable map such that $f^{-1}(D)$ consists of a finite number of points. We would like to understand when this can be lifted to a stable log map. We know that if $\underline{f}$ lifts to a stable log map, then $f^{*} \overline{\mathcal{M}}_{X}$ can only jump at marked and double points, by Remark 1.9, and hence every point of $\underline{f}^{-1}(D)$ must be marked. So we should consider the situation

$$
\underline{f}:\left(\underline{C},\left(x_{1}, \ldots, x_{d}, y_{1}, \ldots, y_{p}\right)\right) \longrightarrow \underline{X}
$$

with $f^{-1}(D)=\left\{x_{1}, \ldots, x_{d}\right\}$.

What is the possible type of a log lifting? Since $\underline{C}$ has no double points, the only relevant information is the choice of $u_{x_{i}} \in \mathbb{N}^{\vee}=\mathbb{N}$, where $\mathbb{N}=\overline{\mathcal{M}}_{X, f\left(x_{i}\right)}$. (The $u_{y_{i}}$ 's are necessarily zero as they lie in the zero monoid.) Furthermore, $P_{\eta}=0$ for $\eta$ the generic point of $\underline{C}$, so once the type is chosen, necessarily $Q=0$. Thus $\mathcal{M}_{C}$ is just the divisorial $\log$ structure on $\underline{C}$ associated with the divisor $x_{1}+\cdots+y_{p}$.

The only constraint on the choice of $u_{x_{i}}$ comes from the balancing condition. More precisely, note that the torsor coming from $n \in \Gamma\left(\underline{X}, \overline{\mathcal{M}}_{X}\right)=\mathbb{N}$ corresponds to the line bundle $\mathcal{O}_{\underline{X}}(-n D)$. Thus in the notation of (1.10), $\tau_{\eta}^{X}: \Gamma\left(\underline{C}, f^{*} \overline{\mathcal{M}}_{X}\right)=$ $\mathbb{N}^{d} \rightarrow \mathbb{Z}$ is given by $\tau_{\eta}^{X}\left(n_{1}, \ldots, n_{d}\right)=-\sum \mu_{i} n_{i}$, where $\mu_{i}>0$ is the order of tangency of $D$ with $\underline{C}$ at the point $x_{i}$. On the other hand, the map $\tau_{\eta}^{C}: \Gamma\left(\underline{C}, \overline{\mathcal{M}}_{C}\right)=$ $\mathbb{N}^{d+p} \rightarrow \mathbb{Z}$ is given by $\tau_{\eta}^{C}\left(n_{1}, \ldots, n_{d}, m_{1}, \ldots, m_{p}\right)=-\sum n_{i}-\sum m_{j}$. Since the map $\varphi: \Gamma\left(\underline{C}, \underline{f}^{*} \overline{\mathcal{M}}_{X}\right) \rightarrow \Gamma\left(\underline{C}, \overline{\mathcal{M}}_{C}\right)$ induced by $\overline{f^{b}}$ is given by

$$
\varphi\left(n_{1}, \ldots, n_{p}\right)=\left(u_{x_{1}} n_{1}, \ldots, u_{x_{d}} n_{d}, 0, \ldots, 0\right),
$$

the only way that $\tau_{\eta}^{C} \circ \varphi$ can coincide with $\tau_{\eta}^{X}$ is if $u_{x_{i}}=\mu_{i}$ for $1 \leq i \leq d$. Thus we see that the elements $u_{x_{i}} \in \mathbb{N}^{\vee}$ can be interpreted as imposing the orders of tangency.

Note that once $u_{x_{i}}=\mu_{i}$ for each $i$, there is a unique map $f^{*} \mathcal{M}_{X} \rightarrow \mathcal{M}_{C}$ induced by $f^{*}: f^{-1} \mathcal{O}_{\underline{X}} \rightarrow \mathcal{O}_{\underline{C}}$, as in this case the structure maps for the log structures embed $f^{*} \mathcal{M}_{X}$ and $\mathcal{M}_{C}$ in $\mathcal{O}_{\underline{C}}$. Thus the open substack of the moduli space of stable log maps corresponding to curves considered in this example coincides with the corresponding substack (not necessarily open now) of the moduli of ordinary stable maps consisting of stable maps as above with the correct orders of tangencies, and the log structure is trivial.

Example 7.2. Continuing with the case of a pair $(\underline{X}, D)$ as in the previous example, it is not difficult to obtain interesting examples for the monoid $Q$. Consider the case that $\underline{X}=\mathbb{P}^{1}, D=\{0\}$, and consider the limiting situation in Figure 7.1 . The figure on the left shows a rational curve with marked points $p_{1}, p_{2}, p_{3}$ mapping $2: 1$ to $\mathbb{P}^{1}$, with order of tangencies to $D$ at the three marked points being 0,0 and 2 , respectively. Such a situation can degenerate to the stable marked curve $f$ pictured to the right, in which all but the two horizontal components map to 0. By semi-stable reduction for stable log maps, there is a limiting stable log map, with some associated monoid $Q$. Let us determine what the type of this curve is and what $Q$ is. 

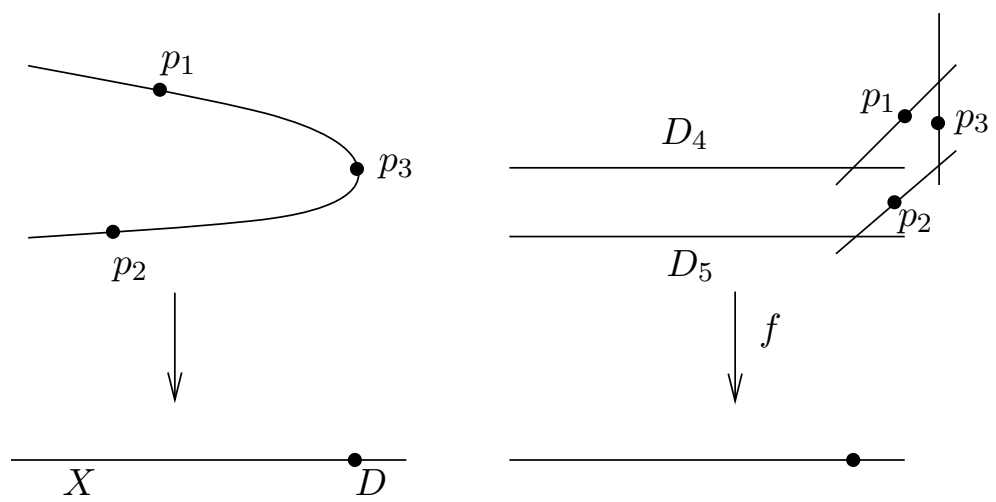

FiguRE 7.1

Let $D_{i}, i=1,2,3$ be the irreducible components of the degenerate domain curve containing the points $p_{i}, i=1,2,3$, respectively. Let $D_{4}$ and $D_{5}$ be the two horizontal components, with $D_{4} \cap D_{1} \neq \emptyset$ and $D_{5} \cap D_{2} \neq \emptyset$. Denote by $\eta_{i}$ the generic point of $D_{i}$. Because $\underline{f}\left(D_{i}\right)=\{0\}, i=1,2,3$, we have $\tau_{\eta_{i}}^{X}=0$ for these $i$, but $\tau_{\eta_{4}}^{X}=\tau_{\eta_{5}}^{X}: \mathbb{N} \rightarrow \mathbb{Z}$ is given by $n \mapsto-n$, as in Example 7.1, We have the four double points

$$
q_{1}=D_{4} \cap D_{1}, \quad q_{2}=D_{1} \cap D_{3}, \quad q_{3}=D_{2} \cap D_{3}, \quad q_{4}=D_{5} \cap D_{2} .
$$

To determine the type of the central fibre, one can use the method of the proof of Proposition 4.3. In particular, necessarily $u_{p_{1}}=u_{p_{2}}=0 \in \mathbb{N}$ and $u_{p_{3}}=$ $2 \in \mathbb{N}$. However, to determine the $u_{q_{i}}$, it is easier to use the balancing condition Proposition 1.15 to observe that there is only one choice of type for the central fibre with these $u_{p_{i}}$. Indeed, suppose we give the central fibre the structure of a stable log map over the standard log point as in 1.4 . This means, in particular, we have data $V_{\eta_{1}}, \ldots, V_{\eta_{5}}$ with $V_{\eta_{1}}, V_{\eta_{2}}, V_{\eta_{3}} \in \mathbb{N}^{\vee}$ and $V_{\eta_{4}}, V_{\eta_{5}}=0$, as well as positive integers $e_{q_{1}}, \ldots, e_{q_{4}}$. Suppose the $u_{p_{i}}$ 's are as given above. If we define the $u_{q_{i}}$ 's by the choice of signs

$$
\begin{array}{ll}
u_{q_{1}}=\left(V_{\eta_{1}}-V_{\eta_{4}}\right) / e_{q_{1}}, & u_{q_{2}}=\left(V_{\eta_{3}}-V_{\eta_{1}}\right) / e_{q_{2}}, \\
u_{q_{3}}=\left(V_{\eta_{3}}-V_{\eta_{2}}\right) / e_{q_{3}}, & u_{q_{4}}=\left(V_{\eta_{2}}-V_{\eta_{5}}\right) / e_{q_{4}},
\end{array}
$$

then by Proposition 1.15 necessarily $u_{q_{i}}=1 \in \mathbb{N}^{\text {gp }}$ for all $i$. Then the monoid $Q$ associated to this type is $\left(P_{\eta_{1}} \oplus P_{\eta_{2}} \oplus P_{\eta_{3}} \oplus \mathbb{N}^{4}\right) / R_{\mathbf{u}}$, where $R_{\mathbf{u}}$ is generated by

$$
\begin{aligned}
& a_{q_{1}}(1)=(-1,0,0,1,0,0,0), \\
& a_{q_{2}}(1)=(1,0,-1,0,1,0,0), \\
& a_{q_{3}}(1)=(0,1,-1,0,0,1,0), \\
& a_{q_{4}}(1)=(0,-1,0,0,0,0,1) .
\end{aligned}
$$

By eliminating the last four components using these relations, $\mathbb{Z}^{7} / R_{\mathbf{u}}$ can be identified with $\mathbb{Z}^{3}$, with the monoid $\mathbb{N}^{7}$ having image in $\mathbb{Z}^{3}$ being generated by $e_{1}=(1,0,0), e_{2}=(-1,0,1), e_{3}=(0,-1,1)$ and $e_{4}=(0,1,0)$. Thus $Q$ is the monoid generated by $e_{1}, e_{2}, e_{3}, e_{4}$ subject to the relation $e_{1}+e_{2}=e_{3}+e_{4}$. This is the monoid defining the quadric cone in $\mathbb{A}^{4}$. 


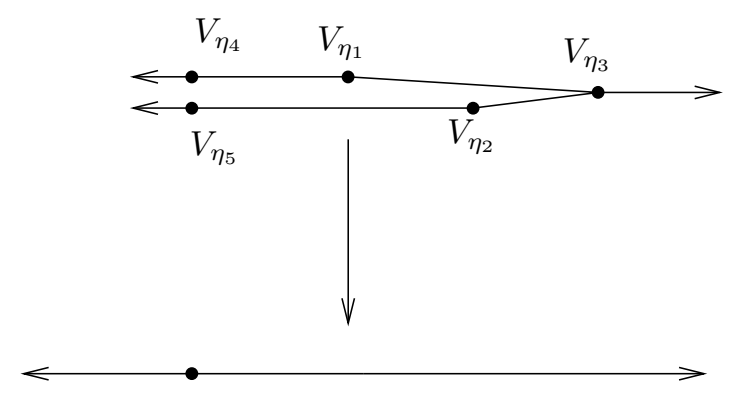

Figure 7.2. The tropical curve associated to $f$. Here the range is $\operatorname{Hom}\left(\Gamma\left(\underline{C}, \underline{f}^{*} \overline{\mathcal{M}}_{X}\right)^{\mathrm{gp}}, \mathbb{R}\right)$.

Note the choice of a map $Q \rightarrow \mathbb{N}$ yields a tropical curve in $\left(\Gamma\left(\underline{C}, f^{*} \overline{\mathcal{M}}_{X}\right)^{\mathrm{gp}}\right)^{*}$, as depicted in Figure 7.2 ,

Example 7.3. Let us return to the situation of Example 7.2 comparing our picture with the expanded degeneration picture as described in Section 6. In our picture, the degenerate curve $f$ corresponds to one point in the moduli space. On the other hand, there are three combinatorially distinct possible limits in the expanded degeneration picture, as depicted in Figure 7.3. In Cases I and II, one must insert additional irreducible components into the limit curve $C$ : these components are contracted in our picture, as they are not stable components. Roughly speaking, these two cases correspond, in our language, to curves over $(\operatorname{Spec} \kappa, \mathbb{N})$ with $V_{\eta_{1}}<$ $V_{\eta_{2}}$ and $V_{\eta_{1}}>V_{\eta_{2}}$, respectively. In Case III, however, the limit domain is the same as ours, but note there is still some actual moduli. Indeed, the first bubble component of the range has four special points: the two double points of the range contained in this component, and the images of the marked points $P_{1}$ and $P_{2}$. The cross-ratio of these four points provides a one-parameter moduli space, a copy of $\mathbb{G}_{m}$. Cases I and II can be viewed as limit points in this one-parameter moduli space. So the map $\mathbf{M}(\underline{X} / D, \beta) \rightarrow \mathscr{M}(X, \beta)$ contracts a $\mathbb{P}^{1}$ to a point.

This suggests that in fact $\mathbf{M}(\underline{X} / D, \beta)$ is, in this case, only birational onto its image in $\mathscr{M}(X, \beta)$. We conjecture this is the case in general. In this case, this morphism gives a small resolution of an ordinary double point in the three-dimensional moduli space of basic stable log maps of the type being considered here. Nevertheless, one may conjecture that the log Gromov-Witten invariants defined here coincide with relative Gromov-Witten invariants as defined by Jun Li. We shall leave it to others to prove such a conjecture 16 .

Example 7.4. Next let us consider $\underline{X}=\mathbb{P}^{2}$ with $D \subset \underline{X}$ a union of two distinct lines $L_{1}, L_{2}$. This is a normal crossings divisor, and is the first case that the classical form of relative Gromov-Witten invariants does not cover. Consider the moduli space of degree two stable log rational curves in $\mathbb{P}^{2}$ with four marked points $p_{i j}, 1 \leq i, j \leq 2$, with $p_{i j}$ a point with tangency of order 1 with $L_{i}$ and tangency of order 0 with $L_{3-i}$. The generic case just consists of a conic intersecting $D$ at four distinct smooth points of $D$. For such curves, the existence of the double point in $D$ is irrelevant, and the analysis of Example 7.1 still applies.

\footnotetext{
${ }^{16}$ Note added in final revision: This conjecture has been verified in AMW.
} 


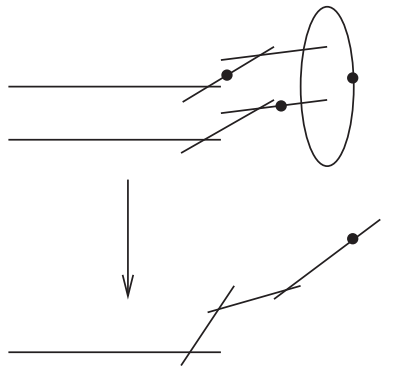

Case I

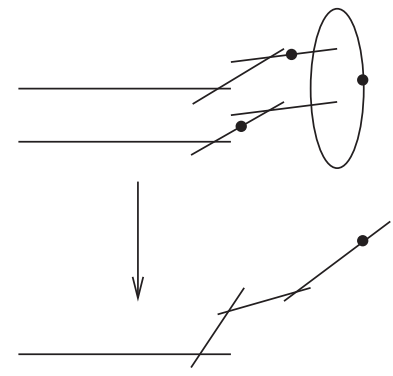

Case II

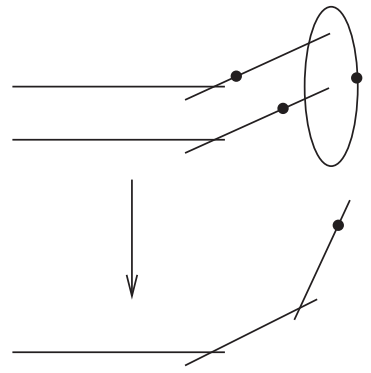

Case III

FiguRE 7.3
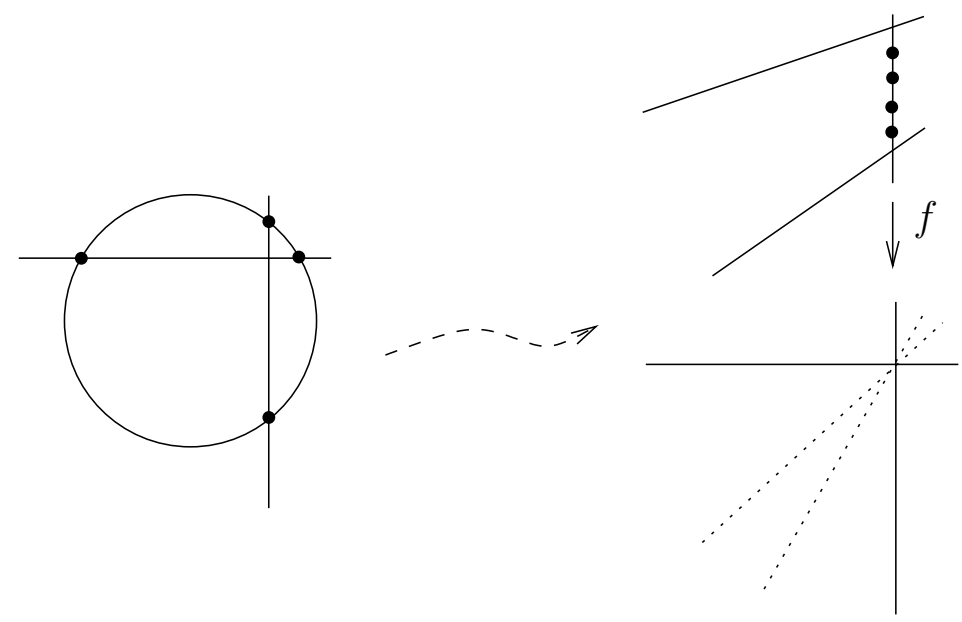

Figure 7.4. The conic on the left degenerates to the reducible curve on the right; the dotted lines are the image of the stable map.

Now consider a limiting curve in which the image of the conic degenerates to a reducible conic $F$ with $F \cap D=L_{1} \cap L_{2}$, that is, the double points of $F$ and $D$ coincide. By stable reduction, there is a limiting stable map $f$, which in the simplest case is given in Figure 7.4. Furthermore, by stable log reduction, this gives a stable log map. Let us determine its type.

First note that $\Gamma\left(\underline{C}, \underline{f}^{*} \overline{\mathcal{M}}_{X}\right)=\mathbb{N}^{2}$. Let the irreducible components of $\underline{C}$ be $D_{1}, D_{2}, D_{3}$ with $D_{3}$ the contracted component. Then necessarily $u_{p_{1 j}}=(1,0)$ and $u_{p_{2 j}}=(0,1)$ for $j=1,2$. One can think of this as saying that even though the $p_{i j}$ 's map into the intersection of the irreducible components, $u_{p_{i j}}$ remembers which component the curve should be tangent to at $p_{i j}$.

As in Example 7.2, we can determine the $u_{q}$ 's by considering the structure of a stable log map over the standard log point. Necessarily $V_{\eta_{1}}=V_{\eta_{2}}=0$. Letting $q_{i}=D_{i} \cap D_{3}, i=1,2$, there is no choice but for $u_{q_{i}}=(1,1)$ (with the proper choice 
of order) in order to achieve the balancing condition at $V_{\eta_{3}}$. One sees in this case that $Q=\mathbb{N}$, with an element of $Q^{\vee}$ specifying $e_{q_{1}}=e_{q_{2}}$.

Example 7.5. Let $\underline{X}$ be a complete toric variety and let $\partial \underline{X} \subset \underline{X}$ be the toric boundary, the union of toric divisors on $\underline{X}$. Let $\underline{X}$ be given the divisorial $\log$ structure induced by this divisor. In general, we do not expect that $\overline{\mathcal{M}}_{X}$ will be generated by global sections; however, it is easy to see that if $M$ is the character lattice of the torus acting on $X$, then there is a surjection $\underline{M} \rightarrow \overline{\mathcal{M}}_{X}^{\text {gp }}$, as each stalk of $\overline{\mathcal{M}}_{X}^{\mathrm{gp}}$ is a quotient of $M$. In particular, given a stable $\log \operatorname{map}(C /(\operatorname{Spec} \kappa, \mathbb{N}), \mathbf{x}, f)$ with target $X$, by Discussion 1.13 one obtains a balanced tropical curve $h: \Gamma_{\underline{C}} \rightarrow$ $N_{\mathbb{R}}=\operatorname{Hom}(M, \mathbb{R})$. In general, this tropical curve contains one unbounded edge for each irreducible component $D=\operatorname{cl}(\eta)$ of $\underline{C}$ in the direction of $\tau_{\eta}^{X}$, or rather, the image of $\tau_{\eta}^{X}$ in $N=\operatorname{Hom}(M, \mathbb{Z})$. More specifically, given the component $D$ and its normalization $g: \tilde{D} \rightarrow D$, one has a composition

$$
M \longrightarrow \Gamma\left(\tilde{D}, g^{*} \underline{f}^{*} \overline{\mathcal{M}}_{X}^{\mathrm{gp}}\right) \stackrel{\tau_{\eta}^{X}}{\longrightarrow} \mathbb{Z},
$$

which yields the image of $\tau_{\eta}^{X}$ in $N$. Note, however, that in the present toric case the composed map $M \rightarrow \Gamma\left(\underline{X}, \overline{\mathcal{M}}_{X}^{\mathrm{gp}}\right) \rightarrow \operatorname{Pic} X$ is the zero map, as the divisor of zeroes and poles of a monomial $z^{n}$ is linearly equivalent to zero. Hence the image of $\tau_{\eta}^{X}$ in $N$ is in fact zero.

Thus the construction of Discussion 1.13 yields an ordinary tropical curve in $N_{\mathbb{R}}$ whose only unbounded edges necessarily correspond to marked points of the curve $\underline{C}$, and the balancing condition of Proposition 1.15 gives the usual tropical balancing condition in $N_{\mathbb{R}}$. Furthermore, there is a relationship between this tropical curve and the fan $\Sigma$ in $N_{\mathbb{R}}$ for $X$. Indeed, suppose that for an irreducible component $D=\operatorname{cl}(\eta)$ of $\underline{C}$, the toric stratum of $X$ containing $f(\eta)$ corresponds to a cone $\tau \in \Sigma$. Then $h\left(v_{\eta}\right) \in \tau$. Indeed, we have the composition

$$
M \longrightarrow \Gamma\left(\underline{C}, \underline{f}^{*} \overline{\mathcal{M}}_{X}^{\mathrm{gp}}\right) \rightarrow P_{\eta}^{\mathrm{gp}},
$$

with $P_{\eta}=\left(\tau^{\vee} \cap M\right) /\left(\tau^{\vee} \cap M\right)^{\times}$. Thus we have the dual map

$$
P_{\eta}^{\vee} \hookrightarrow\left(P_{\eta}^{\mathrm{gp}}\right)^{*} \longrightarrow N
$$

which identifies $P_{\eta}^{\vee}$ with $\tau \cap N$. As $h\left(v_{\eta}\right)$ is the image of $V_{\eta} \in P_{\eta}^{\vee}$ under this map, one sees $h\left(v_{\eta}\right) \in \tau$.

Note further that the tropical curve $h: \Gamma_{\underline{C}} \rightarrow N_{\mathbb{R}}$ in fact determines the type of the curve, as the maps $P_{x}^{\vee} \rightarrow N$ are injective for any $x \in|\underline{C}|$. However, given an ordinary stable map $f: \underline{C} \rightarrow \underline{X}$, it is not true that any type of tropical curve $h: \Gamma_{\underline{C}} \rightarrow N_{\mathbb{R}}$ with $h\left(v_{\eta}\right)$ lying in the correct cone of $\Sigma$ is an allowable type of log map. Indeed, the balancing condition holding in $N_{\mathbb{R}}$ does not imply the balancing condition of Proposition 1.15.

Figure 7.5 gives an example of a stable map $\underline{f}: \underline{C} \rightarrow \mathbb{P}^{2}$ and a corresponding possible tropical curve. It is not difficult to see that $Q=\mathbb{N}^{5}$ here. 

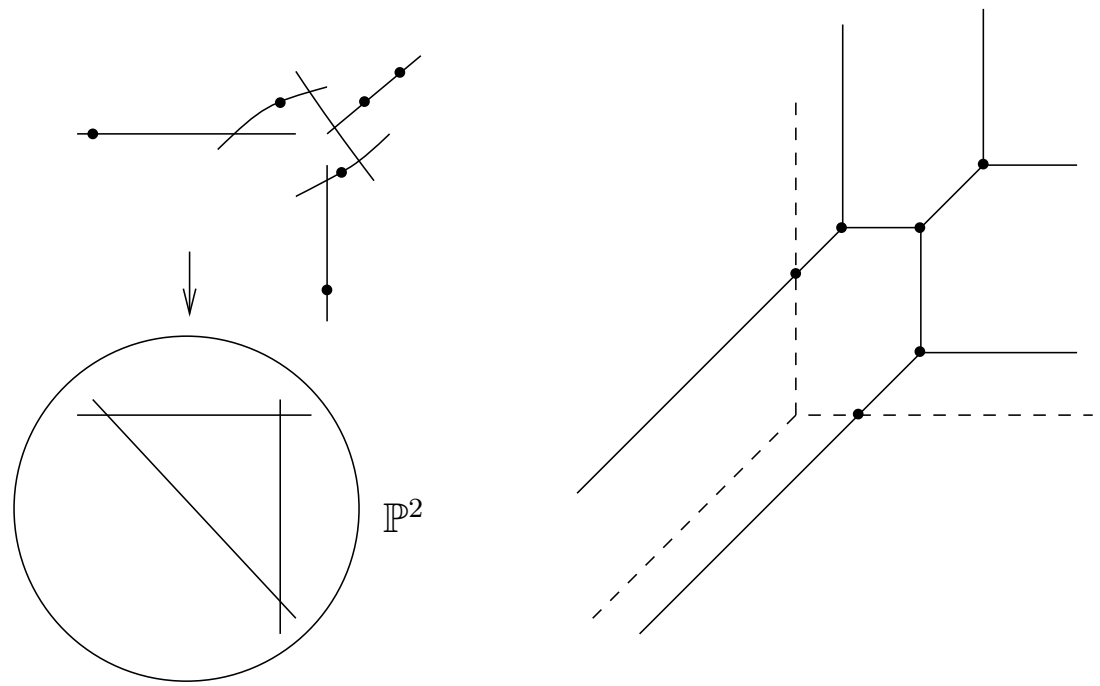

Figure 7.5. The figure on the left depicts the underlying stable map, with marked points as indicated. All components are contracted but the left-hand and lower components, which map to coordinate lines. The right-hand figure shows a possible corresponding tropical curve, with the dotted lines indicating the fan for $\mathbb{P}^{2}$.

\section{Appendix A. The log StaCk of PREstable CURVES}

In this appendix we will sketch the argument that the stack $\mathscr{M}=\mathscr{M}_{S}$ of prestable log smooth curves defined over $S=\left(\underline{S}, \mathcal{M}_{S}\right)$ is an algebraic log stack locally of finite type over $S$. We note that this will be an algebraic stack only in the weaker sense of $\mathrm{Ol2}$, in the sense that the diagonal morphism will not be separated, due to the phenomenon of Example 2.10 inherent in log moduli problems.

We begin by considering the stack $\mathbf{M}$ of ordinary pre-stable curves over $\underline{S}$, that is, for a scheme $V$,

$$
\mathbf{M}(V)=\{(C / V, \mathbf{x}) \text { is a pre-stable curve }\} .
$$

Denote by $\mathbf{M}_{g, k}$ the Deligne-Mumford stack of stable curves of genus $g$ with $k$ marked points [DeMu, $[\mathrm{Kd}]$.

Lemma A.1. $\mathbf{M}$ is an algebraic stack, locally of finite type over $\underline{S}$.

Proof. The fact that the diagonal $\Delta: \mathbf{M} \rightarrow \mathbf{M} \times{ }_{S} \mathbf{M}$ is representable follows from the fact that given two pre-stable curves $\left(C_{1} / V, \mathbf{x}_{1}\right),\left(C_{2} / V, \mathbf{x}_{2}\right)$, the isomorphism functor

$$
\operatorname{Isom}_{V}\left(\left(C_{1} / V, \mathbf{x}_{1}\right),\left(C_{2} / V, \mathbf{x}_{2}\right)\right)
$$

is representable by a closed subscheme of $\operatorname{Isom}_{V}\left(C_{1}, C_{2}\right)$, which in turn is representable by an open subscheme of the Hilbert scheme of $C_{1} \times_{V} C_{2}$ by [Gt]. In particular, $\Delta$ is separated and quasi-compact.

To see that $\mathbf{M}$ has a smooth cover, denote by $\mathbf{M}_{g, k}^{o}$ the open subscheme of $\mathbf{M}_{g, k}$ whose points parameterize stable marked curves with no non-trivial automorphisms. 
Consider the map

$$
p: \coprod_{g, k, \ell \geq 0} \mathbf{M}_{g, k+\ell}^{o} \rightarrow \mathbf{M}
$$

which takes a curve $\left(C / V, x_{1}, \ldots, x_{k+\ell}\right)$ to the curve $\left(C / V, x_{1}, \ldots, x_{k}\right)$. Let $C^{o}$ be the complement of the critical points of $C \rightarrow V$ and let $\left(C^{o}\right)^{\ell}$ denote $C^{o} \times_{V}$ $\cdots \times{ }_{V} C^{o}(\ell$ copies $)$. Given any map $V \rightarrow \mathbf{M}$ corresponding to a pre-stable curve $\left(C / V, x_{1}, \ldots, x_{k}\right)$, one sees easily that $V \times_{\mathbf{M}} \coprod_{g, k, \ell} \mathbf{M}_{g, k+\ell}^{o}$ is represented by an open subscheme of $\coprod_{\ell \geq 0}\left(C^{o}\right)^{\ell}$, and hence $p$ is smooth. On the other hand, $p$ is clearly surjective, since given any pre-stable curve over a separably closed field, one can always add enough marked points so that its automorphism group becomes trivial.

Since $\mathbf{M}_{g, k}$ is of finite type for each $g$ and $k$, this shows $\mathbf{M}$ is an algebraic stack locally of finite type.

Now let us incorporate log structures. Given a pre-stable curve $(\underline{C} / \underline{W}, \mathbf{x})$, the argument of [Kf], p. 227ff, constructs canonical log structures and a log morphism $\left(\underline{C}, \mathcal{M}_{C}\right) \rightarrow\left(\underline{W}, \mathcal{M}_{W}\right)$, with the log structure on $\underline{W}$ called basic. While the result is stated there for stable curves, stability is not used. The point of the basicness property is the following:

Proposition A.2. Given $(C / W, \mathbf{x})$ basic in the above sense, and a pre-stable marked $\log$ curve $(D / Z, \mathbf{y})$ with maps $\underline{\alpha}: \underline{Z} \rightarrow \underline{W}$ and $\beta: \underline{D} \rightarrow \underline{C}$ inducing an isomorphism $\underline{D} \rightarrow \underline{C} \times \underline{W} \underline{Z}$, there exist unique maps $\alpha: \bar{Z} \rightarrow W$ and $\beta: D \rightarrow C$ with underlying scheme morphisms $\underline{\alpha}, \underline{\beta}$ such that the diagram

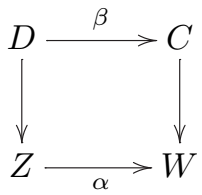

is cartesian.

Proof. This is the content of Proposition 2.1 and Theorem 2.1 of [ Kf]. The result as stated does not rely on stability of the curves.

In particular, basicness is stable under base change, that is, given $\underline{W^{\prime}} \rightarrow \underline{W}$, the basic log structure on $\left(\underline{C} \times_{W} \underline{W^{\prime}} / \underline{W^{\prime}}, \mathbf{x}\right)$ is the pull-back of the basic log structure on $(\underline{C} / \underline{W}, \mathbf{x})$. This endows $\mathbf{M}$ with a log structure,

$$
\mathbf{M} \longrightarrow(\mathrm{Log} / S) \longrightarrow(\mathrm{Sch} / \underline{S}),
$$

thus generalizing (1.2).

Finally we want to allow arbitrary log structures on the base. For this we use Olsson's deep result that the stack $\log _{S}$ of fine $\log$ schemes over $S$ is algebraic (but not with a separated diagonal) and locally of finite type over $\underline{S}$ ([12, Theorem 1.1). Similarly, there is an algebraic stack $\log _{S}^{\bullet \rightarrow \bullet}$ of schemes $\underline{T}$ over $\underline{S}$ together with a morphism of fine $\log$ structures $\mathcal{M}_{1} \rightarrow \mathcal{M}_{2}$ on $T$ ([Ol3], Example 2.1; this is a direct consequence of $\mathrm{Ol2}$, Proposition 5.9). The forgetful functor to the first log 
structure $\mathcal{M}_{1}$ defines a morphism

$$
\mathcal{L}_{\log }^{\bullet \rightarrow \bullet} \longrightarrow \mathcal{L}_{S}
$$

of algebraic stacks. Clearly, $\mathbf{M} \rightarrow(\log / S)$ in fact defines a morphism of algebraic stacks $\mathbf{M} \rightarrow \mathcal{L}_{\operatorname{og}}$. Now the fibre product

$$
\mathcal{L}_{\log }^{\bullet \rightarrow \bullet} \times \times_{\mathcal{L o g}_{S}} \mathbf{M}
$$

is isomorphic to the stack $\mathscr{M}$ of pre-stable log curves (with arbitrary log structures on the base). In fact, an object over $\underline{W} \in(\mathrm{Sch} / \underline{S})$ consists of a basic pre-stable log curve $\left(C /\left(\underline{W}, \mathcal{M}_{W}^{0}\right), \mathbf{x}\right)$ and a morphism of fine log structures $\mathcal{M}_{W}^{0} \rightarrow \mathcal{M}_{W}$. Pulling back thus defines a log curve over $\left(\underline{W}, \mathcal{M}_{W}\right)$, and Proposition A.2 says that this functor defines an isomorphism of stacks from the fibre product to $\mathscr{M}$. Summarizing, we have the following result.

Proposition A.3. The stack $\mathscr{M}$ of pre-stable marked log curves over fine log schemes over $S$ is an algebraic log stack locally of finite type over $\underline{S}$.

\section{Appendix B. Tropicalization of a LOG SPACE}

Traditionally, tropical geometry provides a discrete version of algebraic geometry in $\mathbb{G}_{m}^{n}$ over some valuation ring. An almost equivalent point of view is to work in an equivariant compactification, thus replacing $\mathbb{G}_{m}^{n}$ by a toric variety. The authors have emphasized at various places that tropical geometry can also be viewed as providing the discrete information captured in the ghost sheaf of a log structure. In the present context we have seen traditional tropical curves to arise from a stable log map $(C /(\operatorname{Spec} \kappa, \mathbb{N}), \mathbf{x}, f)$ over the standard log point provided $\underline{f}^{*} \overline{\mathcal{M}}^{\mathrm{gp}}$ is globally generated (Discussion 1.13). Without global generatedness, a natural target space for a tropical curve might be a space we call the tropicalization of $X$, (analogous to the notion of the tropical part of an exploded manifold of Parker's work $[\mathrm{Pa}]$ ), defined as follows.

Given a $\log$ scheme $X$ with $\log$ structure in the Zariski topology, we set

$$
\operatorname{Trop}(X):=\left(\coprod_{x \in \underline{X}} \operatorname{Hom}\left(\overline{\mathcal{M}}_{X, x}, \mathbb{R}_{\geq 0}\right)\right) / \sim,
$$

where the disjoint union is over all scheme-theoretic points of $\underline{X}$ and the equivalence relation is generated by the identifications of faces given by dualizing generization maps $\overline{\mathcal{M}}_{X, x} \rightarrow \overline{\mathcal{M}}_{X, x^{\prime}}$ when $x$ is specialization of $x^{\prime}$. One then obtains for each $x$ a map

$$
i_{x}: \operatorname{Hom}\left(\overline{\mathcal{M}}_{X, x}, \mathbb{R}_{\geq 0}\right) \rightarrow \operatorname{Trop}(X) .
$$

For general $X, \operatorname{Trop}(X)$ may not be particularly well-behaved, as the equivalence relation might yield strange self-identifications of faces.

Example B.1. Let $X$ be a log scheme whose underlying scheme is a union $C_{1} \cup C_{2}$ of two copies of $\mathbb{P}^{1}$ with generic points $\eta_{1}, \eta_{2}$, meeting at two points $q_{1}, q_{2}$ (hence a degenerate elliptic curve). It is not difficult to find a Zariski log structure such 
that $\overline{\mathcal{M}}_{X, q_{i}} \cong \mathbb{N}^{4}, \overline{\mathcal{M}}_{X, \eta_{i}} \cong \mathbb{N}^{3}$, with generization maps:

$$
\begin{aligned}
& \overline{\mathcal{M}}_{X, q_{1}} \longrightarrow \overline{\mathcal{M}}_{X, \eta_{1}}, \quad \sum_{i} a_{i} e_{i} \longmapsto a_{1} e_{1}+a_{2} e_{2}+a_{3} e_{3}, \\
& \overline{\mathcal{M}}_{X, q_{1}} \longrightarrow \overline{\mathcal{M}}_{X, \eta_{2}}, \quad \sum_{i} a_{i} e_{i} \longmapsto a_{1} e_{1}+a_{2} e_{2}+a_{4} e_{4}, \\
& \overline{\mathcal{M}}_{X, q_{2}} \longrightarrow \overline{\mathcal{M}}_{X, \eta_{1}}, \quad \sum_{i} a_{i} e_{i} \longmapsto a_{1} e_{1}+a_{2} e_{2}+a_{3} e_{3}, \\
& \overline{\mathcal{M}}_{X, q_{2}} \longrightarrow \overline{\mathcal{M}}_{X, \eta_{2}}, \quad \sum_{i} a_{i} e_{i} \longmapsto a_{2} e_{1}+a_{1} e_{2}+a_{4} e_{4} .
\end{aligned}
$$

Then one sees that $\operatorname{Trop}(X)$ is not particularly well-behaved. It is a quotient of a disjoint union of two copies of the orthant generated by $e_{1}^{*}, \ldots, e_{4}^{*}$, with the face generated by $e_{1}^{*}, e_{2}^{*}$ of one of these orthants identified with the same face of the other orthant using two different identifications: the identity and the identification swapping $e_{1}^{*}$ and $e_{2}^{*}$. Hence $\operatorname{Trop}(X)$ cannot be viewed as a polyhedral complex in this case.

In general, it is helpful to view the tropicalization more abstractly as a collection of cones with maps between them. This might provide the correct notion of a tropical stack. This point of view will be explored in more detail elsewhere.

It is sometimes useful to impose a condition which allows us to avoid such a possibility:

Definition B.2. Let $X$ be an fs $\log$ scheme. We say $X$ is monodromy free if for any geometric point $x \in \underline{X}, i_{x}$ is injective on the interior of any face of $\operatorname{Hom}\left(\overline{\mathcal{M}}_{X, x}, \mathbb{R}_{\geq 0}\right)$.

In general, $\operatorname{Trop}(X)$ is a good range space for the tropical curves arising from $\log$ maps. Specifically, given a stable $\log \operatorname{map}(C /(\operatorname{Spec} \kappa, \mathbb{N}), \mathbf{x}, f)$ over a standard $\log$ point, we map $v_{\eta}$ to $i_{f(\bar{\eta})}\left(V_{\eta}\right)$, where now $V_{\eta}$ is viewed as an element of $\operatorname{Hom}\left(P_{\eta}, \mathbb{R}_{\geq 0}\right)$. We map an edge $E_{q}$ to the image of the line segment in $\operatorname{Hom}\left(P_{q}, \mathbb{R}_{\geq 0}\right)$ joining the images of the endpoints of $E_{q}$, as usual. We map $E_{p}$ to a ray with endpoint $i_{f(\bar{\eta})}\left(V_{\eta}\right)$ in the direction $i_{f(\bar{\eta})}\left(u_{p}\right)$, if $p$ is in the closure of $\eta$.

Remark B.3. A more conceptual way to view this construction is to observe that Trop is a covariant functor: given a morphism of Zariski log schemes $f: X \rightarrow Y$, for $x \in X$ we obtain a map $f^{b}: \overline{\mathcal{M}}_{Y, f(x)} \rightarrow \overline{\mathcal{M}}_{X, x}$ and hence a map $\operatorname{Hom}\left(\overline{\mathcal{M}}_{X, x}, \mathbb{R}_{\geq 0}\right) \rightarrow$ $\operatorname{Hom}\left(\overline{\mathcal{M}}_{Y, f(x)}, \mathbb{R}_{\geq 0}\right)$. This is compatible with the equivalence relations defining $\operatorname{Trop}(X)$ and $\operatorname{Trop}(Y)$. Indeed, if $x$ is a specialization of $x^{\prime}, f(x)$ is a specialization of $f\left(x^{\prime}\right)$, and hence inclusions of faces are compatible with the induced maps on cones.

The above description of the tropical curve associated to $(C /(\operatorname{Spec} \kappa, \mathbb{N}), \mathbf{x}, f)$ can be viewed as follows. We have a map $\operatorname{Trop}(\pi): \operatorname{Trop}(C) \rightarrow \operatorname{Trop}(\operatorname{Spec} \kappa, \mathbb{N})=\mathbb{R}_{\geq 0}$, and $\operatorname{Trop}(f): \operatorname{Trop}(\pi)^{-1}(1) \rightarrow \operatorname{Trop}(X)$ is easily seen to coincide with the tropical curve described above.

More generally, suppose we have a basic stable $\log \operatorname{map}(C /(\operatorname{Spec} \kappa, Q), \mathbf{x}, f)$ over a point. Then we obtain a family of tropical curves parameterized by $\operatorname{Hom}\left(Q, \mathbb{R}_{\geq 0}\right)$. In particular, the tropical curves corresponding to pull-backs of this stable log map to standard $\log$ points are given by the restriction of $\operatorname{Trop}(f)$ to $\operatorname{Trop}(\pi)^{-1}(q)$, for $q \in \operatorname{Int}\left(Q^{\vee}\right)$. 


\section{ACKNOWLEDGEMENTS}

The authors thank M. Olsson and R. Pandharipande for valuable discussions, and D. Abramovich, Q. Chen and B. Kim for their interest in this work and for keeping us updated on the progress of their work. The authors also thank one of the referees for a very careful reading of the paper and several valuable comments.

\section{REFERENCES}

[AbCh] D. Abramovich, Q. Chen: Stable logarithmic maps to Deligne-Faltings pairs II, preprint arXiv: 1102.4531 [math.AG], 19pp.

[ACGM] D. Abramovich, Q. Chen, W.D. Gillam, S. Marcus: The evaluation space of logarithmic stable maps, preprint arXiv:1012.5416 [math.AG], 19pp.

[AMW] D. Abramovich, S. Marcus, J. Wise: Comparison theorems for Gromov-Witten invariants of smooth pairs and of degenerations, preprint arXiv:1207.2085 [math.AG], 43pp.

[Ar] M. Artin, Versal deformations and algebraic stacks, Invent. Math. 27 (1974), 165-189. MR0399094 (53 \#2945)

[Be] K. Behrend, Gromov-Witten invariants in algebraic geometry, Invent. Math. 127 (1997), no. 3, 601-617, DOI 10.1007/s002220050132. MR1431140 (98i:14015)

[BeFa] K. Behrend and B. Fantechi, The intrinsic normal cone, Invent. Math. 128 (1997), no. 1, 45-88, DOI 10.1007/s002220050136. MR.1437495 (98e:14022)

[BeMa] K. Behrend and Yu. Manin, Stacks of stable maps and Gromov-Witten invariants, Duke Math. J. 85 (1996), no. 1, 1-60, DOI 10.1215/S0012-7094-96-08501-4. MR1412436 (98i:14014)

[Ch] Q. Chen: Stable logarithmic maps to Deligne-Faltings pairs I, preprint arXiv:1008.3090 [math. AG], 48pp.

[DeMu] P. Deligne and D. Mumford, The irreducibility of the space of curves of given genus, Inst. Hautes Études Sci. Publ. Math. 36 (1969), 75-109. MR0262240 (41 \#6850)

[FuPa] W. Fulton and R. Pandharipande, Notes on stable maps and quantum cohomology, Algebraic geometry - Santa Cruz 1995, Proc. Sympos. Pure Math., vol. 62, Amer. Math. Soc., Providence, RI, 1997, pp. 45-96. MR1492534 (98m:14025)

[Ga] Andreas Gathmann, Absolute and relative Gromov-Witten invariants of very ample hypersurfaces, Duke Math. J. 115 (2002), no. 2, 171-203, DOI 10.1215/S0012-7094-0211521-X. MR 1944571 (2003k:14068)

[Gs] Mark Gross, The Strominger-Yau-Zaslow conjecture: from torus fibrations to degenerations, Algebraic geometry-Seattle 2005. Part 1, Proc. Sympos. Pure Math., vol. 80, Amer. Math. Soc., Providence, RI, 2009, pp. 149-192. MR2483935 (2010b:14080)

[Gt] Alexander Grothendieck, Techniques de construction et théorèmes d'existence en géométrie algébrique. IV. Les schémas de Hilbert, Séminaire Bourbaki, Vol. 6, Soc. Math. France, Paris, 1995, pp. Exp. No. 221, 249-276 (French). MR.1611822

[Il] Luc Illusie, Complexe cotangent et déformations. I, Lecture Notes in Mathematics, Vol. 239, Springer-Verlag, Berlin, 1971 (French). MR0491680 (58 \#10886a)

[IoPa] Eleny-Nicoleta Ionel and Thomas H. Parker, Relative Gromov-Witten invariants, Ann. of Math. (2) 157 (2003), no. 1, 45-96, DOI 10.4007/annals.2003.157.45. MR.1954264 (2004a:53112)

[Kf] Fumiharu Kato, Log smooth deformation and moduli of log smooth curves, Internat. J. Math. 11 (2000), no. 2, 215-232, DOI 10.1142/S0129167X0000012X. MR.1754621 (2001d:14016)

[Kk] Kazuya Kato, Logarithmic structures of Fontaine-Illusie, Algebraic analysis, geometry, and number theory (Baltimore, MD, 1988), Johns Hopkins Univ. Press, Baltimore, MD, 1989, pp. 191-224. MR.1463703 (99b:14020)

[Ki] Bumsig Kim, Logarithmic stable maps, symmetry (RIMS, Kyoto, 2008), Adv. Stud. Pure Math., vol. 59, Math. Soc. Japan, Tokyo, 2010, pp. 167-200. MR2683209 (2011m:14019)

[Kd] Finn F. Knudsen, The projectivity of the moduli space of stable curves. II. The stacks $M_{g, n}$, Math. Scand. 52 (1983), no. 2, 161-199. MR702953(85d:14038a)

[Kt] Donald Knutson, Algebraic spaces, Lecture Notes in Mathematics, Vol. 203, SpringerVerlag, Berlin, 1971. MR0302647 (46 \#1791) 
[Kr] Andrew Kresch, Cycle groups for Artin stacks, Invent. Math. 138 (1999), no. 3, 495-536, DOI 10.1007/s002220050351. MR1719823(2001a:14003)

[LaMB] Gérard Laumon and Laurent Moret-Bailly, Champs algébriques, Ergebnisse der Mathematik und ihrer Grenzgebiete. 3. Folge. A Series of Modern Surveys in Mathematics [Results in Mathematics and Related Areas. 3rd Series. A Series of Modern Surveys in Mathematics], vol. 39, Springer-Verlag, Berlin, 2000 (French). MR1771927(2001f:14006)

[LiRu] An-Min Li and Yongbin Ruan, Symplectic surgery and Gromov-Witten invariants of Calabi-Yau 3-folds, Invent. Math. 145 (2001), no. 1, 151-218, DOI 10.1007/s002220100146. MR.1839289 (2002g:53158)

[Li1] Jun Li, Stable morphisms to singular schemes and relative stable morphisms, J. Differential Geom. 57 (2001), no. 3, 509-578. MR.1882667(2003d:14066)

[Li2] Jun Li, A degeneration formula of $G W$-invariants, J. Differential Geom. 60 (2002), no. 2, 199-293. MR1938113 (2004k:14096)

[LiTi] Jun Li and Gang Tian, Virtual moduli cycles and Gromov-Witten invariants of algebraic varieties, J. Amer. Math. Soc. 11 (1998), no. 1, 119-174, DOI 10.1090/S0894-0347-9800250-1. MR 1467172 (99d:14011)

[NiSi] Takeo Nishinou and Bernd Siebert, Toric degenerations of toric varieties and tropical curves, Duke Math. J. 135 (2006), no. 1, 1-51, DOI 10.1215/S0012-7094-06-13511-1. MR2259922 (2007h:14083)

[Nt] Nitin Nitsure, Construction of Hilbert and Quot schemes, Fundamental algebraic geometry, Math. Surveys Monogr., vol. 123, Amer. Math. Soc., Providence, RI, 2005, pp. 105-137. MR2223407

[Og] A. Ogus: Lectures on logarithmic algebraic geometry. TeXed notes (2006).

[Ol1] Martin Christian Olsson, Log algebraic stacks and moduli of log schemes, ProQuest LLC, Ann Arbor, MI, 2001. Thesis (Ph.D.)-University of California, Berkeley. MR2702292

[Ol2] Martin C. Olsson, Logarithmic geometry and algebraic stacks, Ann. Sci. École Norm. Sup. (4) 36 (2003), no. 5, 747-791, DOI 10.1016/j.ansens.2002.11.001 (English, with English and French summaries). MR2032986(2004k:14018)

[Ol3] Martin C. Olsson, The logarithmic cotangent complex, Math. Ann. 333 (2005), no. 4, 859-931, DOI 10.1007/s00208-005-0707-6. MR2195148(2006j:14017)

[O14] Martin C. Olsson, Deformation theory of representable morphisms of algebraic stacks, Math. Z. 253 (2006), no. 1, 25-62, DOI 10.1007/s00209-005-0875-9. MR2206635 (2006i:14010)

[Pa] Brett Parker, Exploded manifolds, Adv. Math. 229 (2012), no. 6, 3256-3319, DOI 10.1016/j.aim.2012.02.005. MR2900440

[SGA1] Revêtements étales et groupe fondamental, Springer-Verlag, Berlin, 1971 (French). Séminaire de Géométrie Algébrique du Bois Marie 1960-1961 (SGA 1); Dirigé par Alexandre Grothendieck. Augmenté de deux exposés de M. Raynaud; Lecture Notes in Mathematics, Vol. 224. MR0354651 (50 \#7129)

[Si1] Bernd Siebert, Virtual fundamental classes, global normal cones and Fulton's canonical classes, Frobenius manifolds, Aspects Math., E36, Vieweg, Wiesbaden, 2004, pp. 341358. MR2115776 (2005k:14119)

[Si2] B. Siebert: Gromov-Witten invariants in relative and singular cases, Lecture given at the workshop "Algebraic aspects of mirror symmetry", Univ. Kaiserslautern, Germany, June 2001.

[Si3] B. Siebert: Obstruction theories revisited, manuscript 2002.

Department of Mathematics, University of California, San Diego, 9500 Gilman Drive, La Jolla, California 92093-0112

E-mail address: mgross@math.ucsd.edu

FB Mathematik, Universität Hamburg, Bundesstrasse 55, 20146 Hamburg, Germany E-mail address: bernd.siebert@math.uni-hamburg.de 